\title{
Adjunction in 2-categories
}

\author{
D. Kaledin*
}

\section{Contents}

1 Generalities 4

1.1 Categories, functors and equivalences. . . . . . . . . . 4

1.2 Strict 2-categories and 2-functors. . . . . . . . . . . . 7

1.3 Grothendieck construction. . . . . . . . . . . . . . . . . 9

1.4 Cartesian functors. . . . . . . . . . . . . . . . . . . . . . . . . . . . . . .

1.5 Examples. ...................... 14

2 Definitions and statements.

2.1 Nerves and 2-categories. . . . . . . . . . . . 17

2.2 Grothendieck construction for 2-categories. . . . . . . . . . . 21

2.3 Rigidification. . . . . . . . . . . . . . . . . 23

2.4 Monoidal structures. . . . . . . . . . . . . . . . 26

3 Adjunction. 29

3.1 Adjoint pairs. . . . . . . . . . . . . . . . . 29

3.2 Co-adjoint pairs and twisting. . . . . . . . . . . . . . 32

3.3 Iterated adjoint pairs. . . . . . . . . . . . . . 34

4 Applications. $\quad 39$

4.1 Symmetric monoidal structures. . . . . . . . . . . . . . 39

4.2 Morita 2-category. . . . . . . . . . . . . . . 42

4.3 Derived Morita 2-category. . . . . . . . . . . . . . . 46

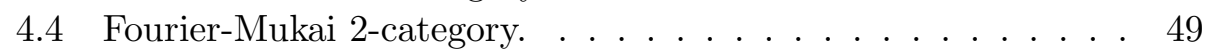

4.5 Equivalences. . . . . . . . . . . . . . 53

${ }^{*}$ Work supported by the Russian Science Foundation, grant 18-11-00141. No funding from any other source. 


\section{Introduction.}

The fact that what categories form is a 2-category rather than simply a category is well-known but usually ignored. It is common to see a commutative diagram of categories and functors, with the tacit understanding that the diagram of course only commutes up to a fixed isomorphism whose exact nature is obvious and left to the reader. This is especially prominent when categories themselves become an object of study, such as for instance in the recent formulations of non-commutative algebraic geometry in the spirit of O, Sections 2,3]. Up to a point, this does not create any problems. However, when the theory becomes more advanced, it can become necessary to spell things out precisely. This is especially the case when adjoint functors and various adjunction maps come into play, such as for instance in the theory of spherical functors of [AL1].

Unfortunately, spelling things out is not easy because the general theory of 2-categories stands on very cumbersome foundations. Since everything only commutes up to an isomorphism, these isomorphisms also have to be carried around, and one has to constantly check the higher-order relations these isomorphisms are supposed to satisfy. Even when one deals with monoidal categories - that is, 2-categories with one object - the resulting combinatorics quickly gets completely out of hand (for a good illustration, see e.g. Chapter VII of the classic textbook $[\mathrm{M}]$ ). Giving a complete treatment of say adjunction in such a language does not look like a thing an actual human can do.

Part of the reason for this situation could be the idea that 2-categories are inherently something that is one level up from usual categories on a certain hypothetical ladder, whose higher levels no-one ever saw, but that is supposed nevertheless to go all the way to the equally hypothetic infinity. Thus theoretically, the theory of 2-categories is supposed to be built from scratch, and its bizarre notation and arcane multilevel commutative diagrams are not a bug but a feature: new combinatorics for a new world. But from the practical viewpoint, this idea looks like a habitual illusion. If one abandons it, then perfectly adequate tools for working with 2-categories are already available within the usual category theory as known and practiced for decades. The first of these is Grothendieck's packaging of "families of categories" into a "Grothendieck fibration" of [G], so conspicuously and inexplicably missing from [M]. This is sometimes known as the "Grothendieck construction", and at the end of the day, it is one of the only two general constructions in abstract category theory that are both non-trivial and useful for a working mathematician (the other one being the notion of a factor- 
ization system of $[\mathrm{Bou}]$ ). The second ingredient is G. Segal's approach to infinite loop spaces introduced in $[\mathrm{S}$ ] that works for categories just as well as, or even better than, for topological spaces.

Combining the two ingredients, one obtains a simple and effective way of packaging 2-categories and related structures (monoidal categories, symmetric monoidal categories) that has been around and ready for use since at least early 1970-ies. In retrospect, it is a pity that such a "Segal-style" approach has not been adopted in the foundational papers on Tannaka formalism such as [DM]. In any case, for quite some time now, the approach has been completely standard and "well-known to the experts" but not universally known. Recently, with the advent of various homotopical enhancements of category theory such as " $\infty$-categories", the situation became both better and worse. On one hand, practitioners of that art certainly only use the Segal approach since in that context, nothing else could possibly work. On the other hand, among the uninitiated, the perception now is that Segal-type approach to 2-categories and monoidal structures is something inextricably linked to fibrant simplicial sets, quasicategories and the like, and the whole thing comes as a take-it-or-leave-it monolith where everything depends on everything else. The perception is utterly wrong but very common.

My own most recent encounter with the 2-category formalism was in the course of work on [K3] that expands and develops the technology of trace theories and trace functors of [K1], with the goal of reaching very concrete applications such as [K2, Section 3]. In [K3], the Segal approach is simply adopted by definition, without any explanations or justifications. However, I feel that it might be useful to have a write-up of how it is related to the more traditional 2-categorical technology. This is what the present paper tries to provide.

The paper is mostly written in an overview style and contains well-known results; the only thing that is possibly at least partially new is the treatment of adjunction in Section 3 that gave the name to the paper. Section 11mostly serves to fix notation and terminology, and to give a brief introduction to the Grothendieck construction with its main examples. Nothing whatsoever is new but we have tried to make the exposition reasonably detailed and self-contained. It would have been better to replace Section 1 with a reference to a suitable textbook on category theory but at present, none exist. We have also tried to be very careful in spelling out various functorialities and compatibility conditions, but we have allowed ourselves the liberty of being quite cavalier about say homological algebra and derived categories used in Example 1.17, and various other things very well served by existing literature. In similar style, Section 2 presents the Segal-style definitions of 
various 2-categorical notions and some related results. In particular, we give a complete proof of the rigidification theorem saying that any 2-category is equivalent to a strict one (for the precise statement, see Theorem 2.12). In Section 3, we treat adjunction. We do prove one result skipped as obvious in [K3, Section 7] (namely, Lemma 3.2), but then we switch gears: for more technical results in Section 3 that might also be new, we replace proofs with precise references to [K3]. What we try to do is to present things in a way that is maybe less canonical than [K3, Section 7] but more down-to-earth. Finally, Section 4 can be treated as one extended example: we illustrate the general theory on one concrete real-life application, that of the derived Morita 2-category of DG algebras over a commutative ring $k$, and the related Fourier-Mukai 2-category of algebraic varieties over $k$ as considered e.g. in [AL2]. The technology used is mostly that of [K3, Section 8] (and it is again not new but "well-known to the experts"). Moreover, we again allow ourselves to gloss over most of the homological details, and we concentrate on checking that various canonical isomorphisms are indeed canonical - or rather, on explaining why in our formalism there is almost nothing that needs to be actually checked. Since our goal is to illustrate the general principle, we do not hesitate to make simplifying assumptions - thus Theorem 2.12 is only stated for small 2-categories, and we only work with schemes of finite type. The extended example, and the paper, ends with a comparison theorem claiming that the Fourier-Mukai 2-category over some Noetherian commutative ring $k$ can be naturally embedded into the derived Morita 2-category over the same ring.

Acknowledgements. I am very grateful to anonymous referees and commentators on the Internet for many valuable corrections and suggestions.

\section{Generalities}

1.1 Categories, functors and equivalences. Recall that by definition, a category $\mathcal{C}$ has a collection of objects $c \in \mathcal{C}$, and a set $\mathcal{C}\left(c, c^{\prime}\right)$ of morphisms for any two objects $c, c^{\prime} \in \mathcal{C}$. Objects can form a set or a proper class; in the former case, the category is called small. We also have a distinguished identity morphism id $=\mathrm{id}_{c} \in \mathcal{C}(c, c)$ for any $c \in \mathcal{C}$, and composition maps - o- : $\mathcal{C}\left(c^{\prime}, c^{\prime \prime}\right) \times \mathcal{C}\left(c, c^{\prime}\right) \rightarrow \mathcal{C}\left(c, c^{\prime \prime}\right)$ for any $c, c^{\prime}, c^{\prime \prime} \in \mathcal{C}$ that are associative and unital: we have

$$
(f \circ g) \circ h=f \circ(g \circ h), \quad \text { id } \circ f=f, \quad f \circ \mathrm{id}=f
$$


for any composable triple of morphisms $f, g, h$. The opposite category $\mathcal{C}^{o}$ has the same objects, identities and compositions as $\mathcal{C}$, but the morphisms are written in the opposite direction: we have $\mathcal{C}^{o}\left(c, c^{\prime}\right)=\mathcal{C}\left(c^{\prime}, c\right)$. For any morphism $f \in \mathcal{C}\left(c, c^{\prime}\right)$, we let $f^{o}$ be the same $f$ but considered as a morphism in the opposite category. An object $o \in \mathcal{C}$ is initial if $\mathcal{C}(o, c) \cong$ pt for any $c \in \mathcal{C}$. If an initial object exists, then it is unique up to a unique isomorphism, and we can add a new initial object $o$ to any category $\mathcal{C}$. We denote the resulting category by $\mathcal{C}^{<}$. Dually, a terminal object $o \in \mathcal{C}$ is an initial object of $\mathcal{C}^{\circ}$, and we can always add a new terminal object to any $\mathcal{C}$ and obtain a category $\mathcal{C}^{>}=\left(\mathcal{C}^{o<}\right)^{o}$.

A functor $\gamma: \mathcal{C} \rightarrow \mathcal{C}^{\prime}$ associates an object $\gamma(c) \in \mathcal{C}^{\prime}$ to any object $c \in \mathcal{C}$, and a map $\mathcal{C}\left(c, c^{\prime}\right) \rightarrow \mathcal{C}^{\prime}\left(\gamma(c), \gamma\left(c^{\prime}\right)\right)$ to any two objects $c, c^{\prime} \in \mathcal{C}$; the maps commute with compositions and send identity morphisms to identity morphisms. A functor $\gamma: \mathcal{C}_{0} \rightarrow \mathcal{C}_{1}$ induces the opposite functor $\gamma^{o}: \mathcal{C}_{0}^{o} \rightarrow$ $\mathcal{C}_{1}^{o}$ between the opposite categories. For any category $\mathcal{C}$, we have natural functors $\mathcal{C} \rightarrow \mathcal{C}^{<}, \mathcal{C} \rightarrow \mathcal{C}^{>}$.

Sets and maps between them form a category denoted Sets. Small categories and functors between them form a category denoted Cat. A category is discrete if all its morphisms are identity maps. A discrete small category is the same thing as a set, and sending a set $S \in$ Sets to itself considered as a discrete category gives an embedding Sets $\subset$ Cat. Alternatively, one can consider the category whose objects are elements $s \in S$, and with exactly one morphism between any two objects. We will denote this category by $e(S)$. Any partially ordered set $J$ can be considered as a category with $J\left(j, j^{\prime}\right)=$ pt if $j^{\prime} \geq j$ and $J\left(j, j^{\prime}\right)=\emptyset$ otherwise. In particular, for any integer $n \geq 0$, we have the totally ordered set $[n]=\{0, \ldots, n\}$, with the usual order. If $n=1$, then [1] is the "single-arrow category" with two objects 0 , 1 and unique non-identity morphism $0 \rightarrow 1$.

Recall that a subcategory $\mathcal{C}^{\prime} \subset \mathcal{C}$ is full if $\mathcal{C}^{\prime}\left(c, c^{\prime}\right)=\mathcal{C}\left(c, c^{\prime}\right)$ for any $c, c^{\prime} \in \mathcal{C}^{\prime}$ - that is, $\mathcal{C}$ and $\mathcal{C}^{\prime}$ have the same morphisms - and we will say that $\mathcal{C}^{\prime} \subset \mathcal{C}$ is dense if they have the same objects. Giving a dense subcategory is equivalent to giving a class $v$ of maps in $\mathcal{C}$ that is closed under compositions and contains all the identity maps. For brevity, we call such classes closed, and we denote the corresponding dense subcategory by $\mathcal{C}_{v} \subset \mathcal{C}$. The minimal closed class is the class Id consisting only of identity maps, and $\mathcal{C}_{\mathrm{ld}} \subset \mathcal{C}$ is the discrete category underlying $\mathcal{C}$. Another useful closed class is the class Iso of all invertible maps.

A morphism, or a map, or a natural transformation $\alpha$ between two functors $\gamma_{0}, \gamma_{1}: \mathcal{C} \rightarrow \mathcal{C}^{\prime}$ is a collection of maps $\alpha(c): \gamma_{0}(c) \rightarrow \gamma_{1}(c), c \in \mathcal{C}$, such that $\alpha\left(c^{\prime}\right) \circ \gamma_{0}(f)=\gamma_{1}(f) \circ \alpha(c)$ for any map $f: c \rightarrow c^{\prime}$ in $\mathcal{C}$. The same data 
can be packaged into a single functor

$$
\gamma:[1] \times \mathcal{C} \rightarrow \mathcal{C}^{\prime}
$$

whose restriction to $0 \times \mathcal{C}$ resp. $1 \times \mathcal{C}$ is identified with $\gamma_{0}$ resp. $\gamma_{1}$. A map $\alpha$ is an isomorphism if so is $\alpha(c)$ for any $c \in \mathcal{C}$, and this happens if and only if the functor (1.2) extends to $e(\{0,1\}) \times \mathcal{C} \supset[1] \times \mathcal{C}$.

For any two categories $\mathcal{C}_{0}, \mathcal{C}_{1}$ and functors $\pi_{0}: \mathcal{C}_{0} \rightarrow \mathcal{C}, \pi_{1}: \mathcal{C}_{1} \rightarrow \mathcal{C}$ to a third category $\mathcal{C}$, we will denote by $\mathcal{C}_{0} \times_{\mathcal{C}} \mathcal{C}_{1}$ the category of triples $\left\langle c_{0}, c_{1}, \alpha\right\rangle$ of objects $c_{0} \in \mathcal{C}_{0}, c_{1} \in \mathcal{C}_{1}$ and an isomorphism $\alpha: \pi_{0}\left(c_{0}\right) \cong \pi_{1}\left(c_{1}\right)$. Note that this notation is somewhat abusive, since even when $\mathcal{C}_{0}, \mathcal{C}_{1}, \mathcal{C}$ are small, $\mathcal{C}_{0} \times_{\mathcal{C}} \mathcal{C}_{1}$ is not the fibered product in Cat: the latter is the subcategory of triples $\left\langle c_{0}, c_{1}, a\right\rangle$ such that $\alpha \in \mathbb{I d}$. Analogously, a functor $\mathcal{C}_{0} \rightarrow \mathcal{C}_{1}$ over $\mathcal{C}$ is a pair $\langle\gamma, \alpha\rangle$ of a functor $\gamma: \mathcal{C}_{0} \rightarrow \mathcal{C}_{1}$ and an isomorphism $\alpha: \pi_{1} \circ \gamma \cong \pi_{0}$, and for any $\mathcal{C} \in$ Cat, we denote by Cat $/ \mathcal{C}$ the category whose objects are small categories $\mathcal{C}^{\prime}$ equipped with a functor $\mathcal{C}^{\prime} \rightarrow \mathcal{C}$, and whose morphisms are functors over $\mathcal{C}$. A functor $\langle\gamma, \alpha\rangle: \mathcal{C}_{0} \rightarrow \mathcal{C}_{1}$ over $\mathcal{C}$ is strict if $\alpha(c) \in \mathrm{Id}$ for any $c \in \mathcal{C}_{0}$ (that is, $\gamma$ strictly commutes with projections to $\mathcal{C}$ ). A functor $\gamma: \mathcal{C}_{0} \rightarrow \mathcal{C}$ induces the pullback functor $\gamma^{*}:$ Cat $/ \mathcal{C} \rightarrow$ Cat $/ \mathcal{C}_{0}$ sending $\mathcal{C}^{\prime} \rightarrow \mathcal{C}$ to $\gamma^{*} \mathcal{C}^{\prime}=\mathcal{C}_{0} \times_{\mathcal{C}} \mathcal{C}^{\prime}$. The fiber $\mathcal{C}_{c}^{\prime}$ of a functor $\mathcal{C}^{\prime} \rightarrow \mathcal{C}$ over an object $c \in \mathcal{C}$ is the subcategory $\mathcal{C}_{c}^{\prime} \subset \mathcal{C}$ spanned by objects $c^{\prime}$ such that $\pi\left(c^{\prime}\right)=c$ and morphisms $f$ such that $\pi(f)=\mathrm{id}_{c}$.

A functor $\gamma: \mathcal{C} \rightarrow \mathcal{C}^{\prime}$ is an equivalence if there exists a functor $\gamma^{\prime}: \mathcal{C}^{\prime} \rightarrow \mathcal{C}$ and isomorphisms $a: \mathrm{Id} \cong \gamma^{\prime} \circ \gamma, a^{\prime}: \gamma \circ \gamma^{\prime} \cong \mathrm{Id}$. This is a condition and not an extra structure, and the triple $\left\langle\gamma^{\prime}, a, a^{\prime}\right\rangle$ can be chosen in a canonical way: if some such triple exists, then $\gamma$ admits a right-adjoint functor $\gamma^{\dagger}$, and the adjunction maps Id $\rightarrow \gamma^{\dagger} \circ \gamma, \gamma \circ \gamma^{\dagger} \rightarrow \mathrm{Id}$ are isomorphisms. We recall that the adjoint functor, if it exists, is unique up to a unique isomorphism. Alternatively, one can take the left-adjoint functor, and the isomorphisms inverse to the adjunction maps.

A functor is an equivalence if and only if it is fully faithful and essentially surjective (that is, surjective on isomorphism classes of objects). The class of all equivalences is closed in Cat, and in practice, one is only interested in categories "up to an equivalence". To make this formal, one can invert all equivalences in Cat. Namely, say that a functor $\gamma: \mathcal{C} \rightarrow \mathcal{C}^{\prime}$ inverts a morphism $f$ in $\mathcal{C}$ if $\gamma(f)$ is invertible in $\mathcal{C}^{\prime}$, and denote by $\overline{\text { Cat }}$ the category of small categories and isomorphism classes of functors between them. We then have the tautological functor $\tau$ : Cat $\rightarrow \overline{\text { Cat }}$ sending a category to itself and a functor to its isomorphism class, and by definition, this functor inverts all equivalences in Cat. 
Lemma 1.1. Any functor $\gamma:$ Cat $\rightarrow \mathcal{C}$ to some category $\mathcal{C}$ that inverts all equivalences factors as

$$
\text { Cat } \stackrel{\tau}{\longrightarrow} \overline{\mathrm{Cat}} \stackrel{\gamma^{\prime}}{\longrightarrow} \mathcal{C},
$$

and the factorization is unique up to a unique isomorphism.

Proof. To prove existence, we can take $\gamma^{\prime}=\gamma$ on objects; we then need to check that $\gamma\left(F_{0}\right)=\gamma\left(F_{1}\right)$ as soon as two functors $F_{0}, F_{1}: I \rightarrow I^{\prime}$ between two categories $I, I^{\prime} \in$ Cat are isomorphic. Indeed, such a pair gives a functor $F: e(\{0,1\}) \times I \rightarrow I^{\prime}$, and the embeddings $i_{l}: I \rightarrow e(\{0,1\}) \times I$ onto $l \times I, l=0,1$ are equivalences with common right-inverse given by the projection $e(\{0,1\}) \times I \rightarrow I$. Therefore $\gamma\left(i_{0}\right)=\gamma\left(i_{1}\right)$, so that $\gamma\left(F_{0}\right)=\gamma\left(F_{1}\right)$. Uniqueness is left to the reader.

1.2 Strict 2-categories and 2-functors. A strict 2-category $\mathcal{C}$ is a "category enriched in Cat": it has objects $c \in \mathcal{C}$, a category of morphisms $\mathcal{C}\left(c, c^{\prime}\right)$ for any two objects $c, c^{\prime} \in \mathcal{C}$, identity objects id $=\mathrm{id}_{c} \in \mathcal{C}(c, c)$, and composition functors $\mathcal{C}\left(c^{\prime}, c^{\prime \prime}\right) \times \mathcal{C}\left(c, c^{\prime}\right) \rightarrow \mathcal{C}\left(c, c^{\prime \prime}\right)$ such that (1.1) holds on the nose. To avoid set-theoretical difficulties, it is prudent to assume that all the categories $\mathcal{C}\left(c, c^{\prime}\right)$ are small. If objects form a set and not a proper class, then $\mathcal{C}$ itself is called small.

For a strict 2-category $\mathcal{C}$, there are two opposite 2 -categories that we call the 1-opposite 2-category $\mathcal{C}^{\iota}$ and the 2-opposite 2-category $\mathcal{C}^{\tau}$. They all have the same objects, and morphism categories $\mathcal{C}^{\iota}\left(c, c^{\prime}\right)=\mathcal{C}\left(c^{\prime}, c\right), \mathcal{C}^{\tau}\left(c, c^{\prime}\right)=$ $\mathcal{C}\left(c, c^{\prime}\right)^{o}$.

A usual category $\mathcal{C}$ is trivially a strict 2-category, with discrete $\mathcal{C}\left(c, c^{\prime}\right)$. The first non-trivial example is the 2-category $\mathcal{C}$ at of small categories: for any two small categories $I, I^{\prime}, \mathcal{C}$ at $\left(I, I^{\prime}\right)$ is the category $\operatorname{Fun}\left(I, I^{\prime}\right)$ of functors from $I$ to $I^{\prime}$. More generally, for any $I \in \mathcal{C}$ at, functors over $I$ also form a category in the obvious way, and we have the 2-category $\mathcal{C}$ at $/ I$ : objects are small categories $\mathcal{C}$ equipped with a functor $\mathcal{C} \rightarrow I$, and $\mathcal{C}$ at $/ I\left(\mathcal{C}, \mathcal{C}^{\prime}\right)$ is the category $\operatorname{Fun}_{I}\left(\mathcal{C}, \mathcal{C}^{\prime}\right)$ of functors from $\mathcal{C}$ to $\mathcal{C}^{\prime}$ over $I$.

Strict 2-functors are defined in a straightforward manner, but for most practical applications, they are too strict. It is more convenient to use the following notion.

Definition 1.2. Assume given strict 2-categories $\mathcal{C}$ and $\mathcal{C}^{\prime}$. A 2-functor $\gamma: \mathcal{C} \rightarrow \mathcal{C}^{\prime}$ is a rule that associates an object $\gamma(c) \in \mathcal{C}^{\prime}$ to any object $c \in \mathcal{C}$, a functor $\gamma_{c, c^{\prime}}: \mathcal{C}\left(c, c^{\prime}\right) \rightarrow \mathcal{C}^{\prime}\left(\gamma(c), \gamma\left(c^{\prime}\right)\right)$ to any pair of objects $c, c^{\prime} \in \mathcal{C}$, an 
isomorphism $\gamma_{c}: \mathrm{id}_{\gamma(c)} \cong \gamma_{c, c}\left(\mathrm{id}_{c}\right)$ to any object $c \in \mathcal{C}$, and an isomorphism $\gamma_{c, c^{\prime}, c^{\prime \prime}}: \mu^{\prime} \circ\left(\gamma_{c^{\prime}, c^{\prime \prime}} \times \gamma_{c, c^{\prime}}\right) \cong \gamma_{c, c^{\prime \prime}} \circ \mu$ to any triple of objects $c, c^{\prime}, c^{\prime \prime} \in \mathcal{C}$, where $\mu$ resp. $\mu^{\prime}$ stands for the composition functors in $\mathcal{C}$ resp. $\mathcal{C}^{\prime}$. For any objects $c, c^{\prime} \in \mathcal{C}$ and morphism $f \in \mathcal{C}\left(c, c^{\prime}\right)$, we must have

$$
\gamma_{c, c, c^{\prime}}\left(f \times \mathrm{id}_{c}\right) \circ\left(\mathrm{id} \circ \gamma_{c}\right)=\mathrm{id}, \quad \gamma_{c, c^{\prime}, c^{\prime}}\left(\mathrm{id}_{c^{\prime}} \times f\right) \circ\left(\gamma_{c^{\prime}} \circ \mathrm{id}\right)=\mathrm{id},
$$

and for any four objects $c, c^{\prime}, c^{\prime \prime}, c^{\prime \prime \prime} \in \mathcal{C}$ and three morphisms $f \in \mathcal{C}\left(c, c^{\prime}\right)$, $f^{\prime} \in \mathcal{C}\left(c^{\prime}, c^{\prime \prime}\right), f^{\prime \prime} \in \mathcal{C}\left(c^{\prime \prime}, c^{\prime \prime \prime}\right)$, the square

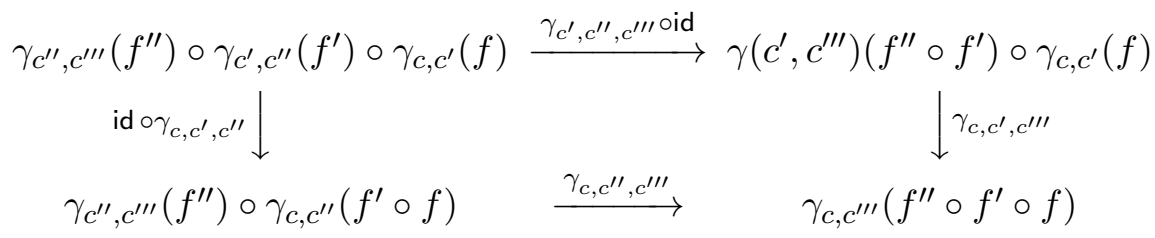

must be commutative.

The collection of 2-functors $\gamma: \mathcal{C} \rightarrow \mathcal{C}^{\prime}$ between strict 2-categories $\mathcal{C}$, $\mathcal{C}^{\prime}$ carries a rich and varied structure: one can define morphisms $\gamma \rightarrow \gamma^{\prime}$ by using (1.2), then morphisms between morphisms, and then all sorts of compositions and associativity constraints. We will need only a small part of this cornucopia, namely, the following.

Definition 1.3. Assume given 2-functors $\gamma, \gamma^{\prime}: \mathcal{C} \rightarrow \mathcal{C}^{\prime}$ between strict 2categories such that $\gamma(c)=\gamma^{\prime}(c)$ for any $c \in \mathcal{C}$. A 2-morphism $\alpha: \gamma \rightarrow \gamma^{\prime}$ is a collection of morphisms $\alpha_{c, c^{\prime}}: \gamma_{c, c^{\prime}} \rightarrow \gamma_{c, c^{\prime}}^{\prime}, c, c^{\prime} \in \mathcal{C}$ such that for any triple of objects $c, c^{\prime}, c^{\prime \prime} \in \mathcal{C}$, the square

$$
\begin{array}{r}
\mu^{\prime} \circ\left(\gamma_{c, c^{\prime}} \times \gamma_{c^{\prime}, c^{\prime \prime}}\right) \stackrel{\gamma_{c, c^{\prime}, c^{\prime \prime}}}{\longrightarrow} \gamma_{c, c^{\prime \prime}} \circ \mu \\
\mu^{\prime} \circ\left(\alpha_{c, c^{\prime}} \times \alpha_{c^{\prime}, c^{\prime \prime}}\right) \downarrow \\
\mu^{\prime} \circ\left(\gamma_{c, c^{\prime}}^{\prime} \times \gamma_{c^{\prime}, c^{\prime \prime}}^{\prime}\right) \stackrel{\alpha_{c, c^{\prime \prime}}}{\stackrel{\gamma_{c, c^{\prime}, c^{\prime \prime}}}{\longrightarrow}} \gamma_{c, c^{\prime \prime}}^{\prime} \circ \mu
\end{array}
$$

is commutative. A 2-morphism $\alpha$ is invertible if so are all the maps $\alpha_{c, c^{\prime}}$.

With this definition, as soon as $\mathcal{C}$ is small, 2-functors $\mathcal{C} \rightarrow \mathcal{C}^{\prime}$ and 2morphisms between them form a category that we denote $\operatorname{Fun}^{2}\left(\mathcal{C}, \mathcal{C}^{\prime}\right)$ (if 2-functors $\gamma, \gamma^{\prime}: \mathcal{C} \rightarrow \mathcal{C}^{\prime}$ do not coincide on objects, there are no maps $\gamma \rightarrow \gamma^{\prime}$ in $\operatorname{Fun}^{2}\left(\mathcal{C}, \mathcal{C}^{\prime}\right)$ ). For any strict 2 -category $\mathcal{C}^{\prime \prime}$, postcomposition with a 2-functor $\mathcal{C}^{\prime} \rightarrow \mathcal{C}^{\prime \prime}$ defines a functor $\operatorname{Fun}^{2}\left(\mathcal{C}, \mathcal{C}^{\prime}\right) \rightarrow \operatorname{Fun}^{2}\left(\mathcal{C}, \mathcal{C}^{\prime \prime}\right)$, and if $\mathcal{C}^{\prime \prime}$ is small, then precomposition with a 2-functor $\gamma: \mathcal{C}^{\prime \prime} \rightarrow \mathcal{C}$ defines a 
functor $\gamma^{*}: \operatorname{Fun}^{2}\left(\mathcal{C}, \mathcal{C}^{\prime}\right) \rightarrow \operatorname{Fun}^{2}\left(\mathcal{C}^{\prime \prime}, \mathcal{C}^{\prime}\right)$. Note that if $\mathcal{C}$ and $\mathcal{C}^{\prime}$ are usual categories considered as 2-categories with discrete categories of morphisms, then $\operatorname{Fun}^{2}\left(\mathcal{C}, \mathcal{C}^{\prime}\right)$ is discrete: objects are functors, but we do not allow any non-trivial maps between them.

Definition 1.4. A 2-functor $\gamma: \mathcal{C} \rightarrow \mathcal{C}^{\prime}$ is a strong equivalence if there exists a 2-functor $\gamma^{\prime}: \mathcal{C}^{\prime} \rightarrow \mathcal{C}$ and invertible 2-morphisms id $\rightarrow \gamma^{\prime} \circ \gamma, \gamma \circ \gamma^{\prime} \rightarrow$ id.

We note that strong equivalences in the sense of Definition 1.4 are indeed rather strong - in particular, objects in $\mathcal{C}$ and $\mathcal{C}^{\prime}$ are canonically the same (and identified by $\gamma$ ). For usual small categories considered as 2-categories, strong equivalence gives an isomorphism in Cat, not just an equivalence.

1.3 Grothendieck construction. Definition 1.2 is a generalization of the notion of a pseudofunctor introduced by Grothendieck in $\mathrm{G}$. A pseudofunctor is a 2-functor from a usual category $I$ to the 2-category $\mathcal{C}$ at of small categories. The great discovery of $[\mathrm{G}$, is that all the data defining a pseudofunctor can be very efficiently packaged by means of the usual category theory. These day, it is known as the Grothendieck construction. Here is a brief summary.

Definition 1.5. A morphism $f: c^{\prime} \rightarrow c$ in a category $\mathcal{C}$ is cartesian with respect to a functor $\pi: \mathcal{C} \rightarrow I$ if any $f^{\prime}: c^{\prime \prime} \rightarrow c$ such that $\pi(f)=\pi\left(f^{\prime}\right)$ uniquely factors as $f^{\prime}=f \circ f_{0}$ with $\pi\left(f_{0}\right)=$ id. A cartesian lifting of a morphism $f: i^{\prime} \rightarrow i$ in $I$ is a morphism $f^{\prime}$ in $\mathcal{C}$ cartesian with respect to $\pi$ and such that $\pi\left(f^{\prime}\right)=f$. A functor $\pi: \mathcal{C} \rightarrow I$ is a prefibration if for any $c \in \mathcal{C}$, any morphism $f: i^{\prime} \rightarrow i=\pi(c)$ in $I$ admits a cartesian lifting $f^{\prime}: c^{\prime} \rightarrow c$. A prefibration is a fibration if the composition of cartesian morphisms is cartesian. A morphism is cocartesian if it is cartesian as a morphism in the opposite category $\mathcal{C}^{o}$ with respect to the opposite functor $\pi^{o}: \mathcal{C}^{o} \rightarrow I^{o}$, and a functor $\pi$ is a cofibration if the opposite functor $\pi^{o}$ is a fibration.

Remark 1.6. For any functor $\pi: \mathcal{C} \rightarrow I$ and object $i \in I$, the right commafiber $i \backslash^{\pi} \mathcal{C}$ is the category of pairs $\langle c, \alpha\rangle$ of an object $c \in \mathcal{C}$ and a map $\alpha: i \rightarrow \pi(c)$. Then by definition, $\mathcal{C}_{i} \subset i \backslash^{\pi} \mathcal{C}$ is the full subcategory spanned by pairs $\langle c, \alpha\rangle$ with $\alpha=\mathrm{id}_{i}$, and $\pi$ is a prefibration if and only for any $i \in I$, the embedding $\mathcal{C}_{i} \rightarrow i \backslash^{\pi} \mathcal{C}$ admits a right-adjoint. In particular, if $i \in I$ is an initial object, then $i \backslash^{\pi} \mathcal{C} \cong \mathcal{C}$, and the embedding $\mathcal{C}_{i} \subset \mathcal{C}$ admits a right-adjoint. Dually, one has a charaterization of cofibrations in terms of 
left comma-fibers $\mathcal{C} /^{\pi} i=\left(i \backslash^{\pi} \mathcal{C}^{o}\right)^{o}$, and if $i \in I$ is terminal, then for any cofibration $\mathcal{C} \rightarrow I$, the embedding $\mathcal{C}_{i} \subset \mathcal{C}$ admits a left-adjoint.

While cartesian liftings in Definition 1.5 are not unique, they are unique up to a unique isomorphism, by virtue of the universal property of cartesian maps. Therefore for any prefibration $\pi: \mathcal{C} \rightarrow I$ and morphism $f: i^{\prime} \rightarrow i$ in $I$, sending $c \in \mathcal{C}_{i}$ to the source $c^{\prime}$ of the cartesian lifting $f: c^{\prime} \rightarrow c$ of the map $f$ defines a functor $f^{*}: \mathcal{C}_{i^{\prime}} \rightarrow \mathcal{C}_{i}$ known as the transition functor of the prefibration. By the same universal property, we have functorial maps

$$
\mathrm{Id} \rightarrow \mathrm{id}_{i}^{*}, \quad g^{*} \circ f^{*} \rightarrow(f \circ g)^{*}
$$

for any $i \in I$ and any composable pair of maps $f, g$ in $I$. The first of these maps is always an isomorphism, and the second is an isomorphism for any $f, g$ if and only if $\pi$ is a fibration. In the latter case, the correspondences $i \mapsto \mathcal{C}_{i}, f \mapsto f^{*}$ and the maps (1.3) define a 2-functor $\gamma: I^{o} \rightarrow \mathcal{C}$ at, with all the conditions of Definition 1.2 again satisfied by virtue of the universal property of cartesian maps. Conversely, for any 2-functor $\gamma: I^{o} \rightarrow \mathcal{C}$ at, we can define a category $\mathcal{C}$ whose objects are pairs $\langle i, c\rangle, i \in I, c \in \mathcal{C}_{i}=\gamma(i)$, and whose morphisms from $\langle i, c\rangle$ to $\left\langle i^{\prime}, c^{\prime}\right\rangle$ are pairs of a map $f: i \rightarrow i^{\prime}$ and a map $c \rightarrow \gamma\left(f^{o}\right)\left(c^{\prime}\right)$. Then the forgetful functor $\mathcal{C} \rightarrow I,\langle i, c\rangle \mapsto i$ is a fibration with transition functors $\gamma\left(f^{o}\right)$. The two constructions are mutually inverse, so they describe the same entity, but the fibration description is much more economical: the only data are the category $\mathcal{C}$ and the functor $\mathcal{C} \rightarrow I$. One does not have to keep track of all the isomorphisms and compatibility conditions of Definition 1.2.

For a cofibration $\mathcal{C} \rightarrow I$, the picture is dual: we have a transition functor $f_{!}: \mathcal{C}_{i} \rightarrow \mathcal{C}_{i^{\prime}}$ for any map $f: i \rightarrow i^{\prime}$ in $I$, and we have the corresponding versions of the isomorphisms (1.3) (both go in the opposite direction, but since they are invertible, this is not so important).

Example 1.7. Assume given two categories $\mathcal{C}_{0}, \mathcal{C}_{1}$, and a functor $\gamma: \mathcal{C}_{0} \rightarrow$ $\mathcal{C}_{1}$. Then the cylinder $\mathrm{C}(\gamma)$ is the category whose objects are those of $\mathcal{C}_{0}$ and of $\mathcal{C}_{1}$, and whose morphisms are given by

$$
\mathrm{C}(\gamma)\left(c, c^{\prime}\right)= \begin{cases}\mathcal{C}_{l}\left(c, c^{\prime}\right), & c, c^{\prime} \in \mathcal{C}_{l}, l=0,1 \\ \mathcal{C}_{1}\left(\gamma(c), c^{\prime}\right), & c \in \mathcal{C}_{0}, c^{\prime} \in \mathcal{C}_{1},\end{cases}
$$

and no morphisms from $c \in \mathcal{C}_{1}$ to $c^{\prime} \in \mathcal{C}_{0}$. We have the natural projection $\mathrm{C}(\gamma) \rightarrow[1]$ with fibers $\mathcal{C}_{0}, \mathcal{C}_{1}$, it is a cofibration with transition functor $\gamma$, and every cofibration $\mathcal{C} \rightarrow$ [1] is of this form. 
By definition, a cofibration $\pi: \mathcal{C} \rightarrow I$ defines the opposite fibration $\pi^{o}: \mathcal{C}^{o} \rightarrow I^{o}$. It also defines the transpose fibration $\mathcal{C}^{\perp} \rightarrow I^{o}$ with fibers $\mathcal{C}_{i}$, transition functors $f^{*}=f_{!}^{o}$, and the isomorphisms (1.3) inverse to the corresponding isomorphisms for $\pi$. Moreover, it might happen that $\pi$ itself is a fibration (it is then called a bifibration). This happens if and only if all the cofibration transition functors $f$ ! admit right-adjoint functors $f^{*}$, and these right-adjoints are then the fibration transition functors. Dually, a fibration $\mathcal{C} \rightarrow I$ might also be a cofibration, and in any case, it defines the opposite cofibration $\mathcal{C}^{o} \rightarrow I^{o}$ and the transpose cofibration $\mathcal{C}_{\perp} \rightarrow I^{o}$. One can also combine the constructions: for any fibration $\mathcal{C} \rightarrow I$, the opposite fibration $\mathcal{C}_{\perp}^{o} \rightarrow I$ to the transpose cofibration $\mathcal{C}_{\perp} \rightarrow I^{o}$ has fibers $\left(\mathcal{C}_{\perp}^{o}\right)_{i}=\mathcal{C}_{i}^{o}$, and transition functors $\left(f^{*}\right)^{o}$, and similarly for cofibrations.

Remark 1.8. The definitions of the transpose fibrations and cofibrations given above use the Grothendieck construction twice, and this is unpleasant since it involves choices. Although choices do cancel out in the end, here is a more direct definition. For any fibration $\pi: \mathcal{C} \rightarrow I$, the transpose cofibration $\mathcal{C}_{\perp}$ has the same objects, and morphisms are isomorphism classes of diagrams

$$
c \stackrel{f}{\longleftarrow} \widetilde{v} \stackrel{v}{\longrightarrow} c^{\prime}
$$

where $f$ is cartesian with respect to $\pi$, and $\pi(v)$ is invertible. Compositions are obtained by taking fibered products, and the existence of the relevant fibered products easily follows from the definition of a fibration. For the transpose fibration $\mathcal{C}^{\perp}$ to a cofibration $\mathcal{C} \rightarrow I$, the arrows in (1.4) go in the other direction, and one uses coproducts rather than products for the compositions.

1.4 Cartesian functors. If we have two fibrations $\mathcal{C}, \mathcal{C}^{\prime} \rightarrow I$, then a functor $\gamma: \mathcal{C} \rightarrow \mathcal{C}^{\prime}$ over $I$ is cartesian over a map $f: i \rightarrow i^{\prime}$ if it sends all cartesian liftings of $f$ to maps in $\mathcal{C}^{\prime}$ cartesian over $I$, and cartesian if it is cartesian over all maps (that is, sends cartesian maps in $\mathcal{C}$ to cartesian maps in $\mathcal{C}^{\prime}$ ). To see this condition more explicitly, it is useful to observe that more generally, if we have two functors $\mathcal{C}, \mathcal{C}^{\prime} \rightarrow I$, and $\mathcal{C}^{\prime} \rightarrow I$ is a fibration resp. a cofibration, then a functor $\langle\gamma, \alpha\rangle$ from $\mathcal{C}$ to $\mathcal{C}^{\prime}$ over $I$ is isomorphic to a strict functor over $I$, unique up to a unique isomorphism and called the strictification of $\gamma$ (this happens because just like any other map, the isomorphisms $\alpha(c), c \in \mathcal{C}$ admit cartesian resp. cocartesian liftings to $\mathcal{C}^{\prime}$ ). If $\mathcal{C}, \mathcal{C}^{\prime} \rightarrow I$ are fibrations, then up to a unique isomorphism, a functor $\gamma: \mathcal{C} \rightarrow \mathcal{C}^{\prime}$ over $I$ is expicitly given by a collection of functors $\gamma(i): \mathcal{C}_{i} \rightarrow \mathcal{C}_{i}^{\prime}$, 
$i \in I$, induced by its strictification, and functorial maps

$$
\gamma(f): \gamma(i) \circ f^{*} \rightarrow f^{*} \circ \gamma\left(i^{\prime}\right)
$$

for all morphisms $f: i \rightarrow i^{\prime}$ in $I$, subject to natural compatibility conditions that we leave to the reader. The functor $\gamma$ is cartesian over $f$ if (1.5) is an isomorphism. If $\mathcal{C}$ is small, then cartesian functors form a full subcategory $\operatorname{Fun}_{I}^{\natural}\left(\mathcal{C}, \mathcal{C}^{\prime}\right) \subset \operatorname{Fun}_{I}\left(\mathcal{C}, \mathcal{C}^{\prime}\right)$ in the category of all functors over $I$, and if we restrict our attension to strict functors and/or strict cartesian functors, we obtain equivalent categories. Fibrations and strict cartesian functors then form a subcategory Cat ${ }^{\natural} / I \subset$ Cat $/ I$ and a 2-subcategory $\mathcal{C} a t^{\natural} / I \subset \mathcal{C}$ at $/ I$, and the 2 -subcategory spanned by all cartesian functors is strongly equivalent to $\mathcal{C} a t^{\natural} / I$.

Dually, for cofibrations, a functor $\gamma: \mathcal{C} \rightarrow \mathcal{C}^{\prime}$ induces functors $\gamma(i)$, $i \in I$, and maps $f_{!} \circ \gamma\left(i^{\prime}\right) \rightarrow \gamma(i) \circ f_{!}$dual to (1.5), unique up to a unique isomorphism, a functor is cocartesian iff all these maps are isomorphisms, and cofibrations together with strict cocartesian functors form a subcategory $\mathrm{Cat}_{\natural} / I \subset$ Cat $/ I$ and a 2-subcategory $\mathcal{C} a t_{\natural} / I \subset \mathcal{C}$ at $/ I$, where in the latter case, dropping "strict" gives a strongly equivalent 2-category. The transpose fibration/transpose cofibration construction provides an equivalence

$$
\mathrm{Cat}^{\natural} / I \cong \mathrm{Cat}_{\natural} / I^{o}
$$

For any fibration $\mathcal{C} \rightarrow I$ and any functor $\gamma: I^{\prime} \rightarrow I$, the pullback $\gamma^{*} \mathcal{C} \rightarrow I^{\prime}$ is a fibration, a pullback of a functor cartesian over $I$ is cartesian over $I^{\prime}$, and similarly for cofibrations and cocartesian functors.

Remark 1.9. As another instance of the strictification phenomenon for fibrations and cofibrations, we note that up to a canonical equivalence, the pullback $\gamma^{*} \mathcal{C}$ of a fibration or a cofibration of small categories is given by an honest fibered product in Cat.

Example 1.10. As a motivation for the transpose cofibration construction, assume given two fibrations $\mathcal{C}, \mathcal{C}^{\prime} \rightarrow I$, and a functor $\gamma: \mathcal{C} \rightarrow \mathcal{C}^{\prime}$ over $I$ such that for any $i \in I$, the component $\gamma(i): \mathcal{C}_{i} \rightarrow \mathcal{C}_{i}^{\prime}$ admits a right-adjoint $\gamma^{\dagger}(i): \mathcal{C}_{i}^{\prime} \rightarrow \mathcal{C}_{i}$. Then for any map $f: i \rightarrow i^{\prime}$ in $I$, the map (1.5) induces by adjunction a map $f^{*} \circ \gamma^{\dagger}\left(i^{\prime}\right) \rightarrow \gamma^{\dagger}(i) \circ f^{*}$, but these maps do not define a functor from $\mathcal{C}^{\prime}$ to $\mathcal{C}$ over $I$ : they go in the wrong direction. What they define is a functor $\gamma_{\perp}^{\dagger}: \mathcal{C}_{\perp}^{\prime} \rightarrow \mathcal{C}_{\perp}$ over $I^{o}$ between the transpose cofibrations. If $\gamma_{\perp}^{\dagger}$ is cocartesian, then it is transpose to a cartesian functor $\gamma^{\dagger}: \mathcal{C}^{\prime} \rightarrow \mathcal{C}$ right-adjoint to $\gamma$. If $\gamma$ is cartesian, then its transpose functor $\gamma_{\perp}: \mathcal{C}_{\perp} \rightarrow \mathcal{C}_{\perp}^{\prime}$ 
is left-adjoint to $\gamma_{\perp}^{\dagger}$. But in general, while $\gamma_{\perp}^{\dagger}$ is defined by $\gamma$ uniquely up to a unique isomorphism, it is not adjoint to $\gamma$ in any obvious sense.

A section of a functor $\mathcal{C} \rightarrow I$ is a functor $I \rightarrow \mathcal{C}$ over $I$, and if $\mathcal{C} \rightarrow I$ is a fibration, a section is cartesian if it sends all maps in $I$ to cartesian maps. If $I$ is small, then sections form a category that we denote by $\operatorname{Sec}(I, \mathcal{C})$, and if $\mathcal{C} \rightarrow I$ is a fibration, we denote by $\operatorname{Sec}^{\natural}(I, \mathcal{C}) \subset \operatorname{Sec}(I, \mathcal{C})$ the full subcategory spanned by cartesian sections. For any subcategory $I^{\prime} \subset I$, with the embeddng functor $j: I^{\prime} \rightarrow I$, we have the restriction functor

$$
\operatorname{Sec}^{\natural}(I, \mathcal{C}) \rightarrow \operatorname{Sec}^{\natural}\left(I^{\prime}, j^{*} \mathcal{C}\right) .
$$

A useful observation is that if $I^{\prime} \subset I$ is full, and the embedding $j$ admits a left-adjoint functor $j^{\dagger}: I \rightarrow I^{\prime}$, then (1.7) is an equivalence. To see this, we may restrict our attention to strict sections. Then the inverse equivalence sends a strict section $\sigma \in \operatorname{Sec}^{\natural}\left(I^{\prime}, j^{*} \mathcal{C}\right)$ to the strict section $\sigma^{\prime} \in \operatorname{Sec}(I, \mathcal{C})$ given by $\sigma^{\prime}(i)=a^{*} \sigma\left(j^{\dagger}(i)\right), i \in I$, where $a: i \rightarrow j\left(j^{\dagger}(i)\right)$ is the adjunction map, with the obvious maps (1.5). Dually, if $\mathcal{C} \rightarrow I$ is a cofibration, we let $\operatorname{Sec}_{\natural}(I, \mathcal{C}) \subset \operatorname{Sec}(I, \mathcal{C})$ be the full subcategory spanned by cocartesian sections, and we have the equivalence (1.7) whenever the full embedding $I^{\prime} \rightarrow I$ admits a right-adjoint.

A composition $\pi^{\prime}=\pi \circ \gamma: \mathcal{C}^{\prime} \rightarrow I$ of two fibrations $\gamma: \mathcal{C}^{\prime} \rightarrow \mathcal{C}, \pi: \mathcal{C} \rightarrow I$ is a fibration but the converse is not true: $\pi^{\prime}$ can be a fibration even if $\gamma$ is not. One has to impose additional conditions; here is a list.

Lemma 1.11. Assume given a fibration $\pi: \mathcal{C} \rightarrow I$ and a functor $\gamma: \mathcal{C}^{\prime} \rightarrow \mathcal{C}$. Then $\gamma$ is a fibration if and only if (i) $\pi^{\prime}=\pi \circ \gamma: \mathcal{C}^{\prime} \rightarrow I$ is a fibration, (ii) $\gamma$ is cartesian over I, (iii) for any $i \in I, \gamma(i)$ is a fibration, and (iv) for any map $f: i \rightarrow i^{\prime}$ in $I$, the functor $\mathcal{C}_{i^{\prime}}^{\prime} \rightarrow\left(f^{*}\right)^{*} \mathcal{C}_{i}^{\prime}$ induced by the commutative diagram

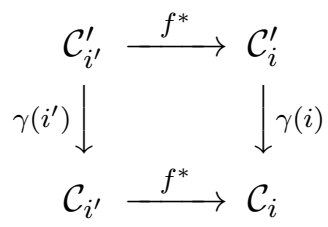

is cartesian over $\mathcal{C}_{i^{\prime}}$.

Proof. The "only if" part is easy and left to the reader. For the "if" part, note that each condition makes sense only if the previous ones are satisfied (in particular, (1.8) in (iv) is commutative by virtue of (ii)). If we let $C$ be 
the class of maps in $\mathcal{C}$ cartesian over $I$, and let $V$ be the class of maps $f$ that are "vertical" in the sense that $\pi(v)$ is invertible, then (i) and (ii) insure that $\gamma$ is a fibration over $\mathcal{C}_{C}$, and (iii) insures that it is a fibration over $\mathcal{C}_{V}$. By the definition of a fibration, every map $f$ in $\mathcal{C}$ factors as $f=c \circ v, v \in V, c \in C$, uniquely up to a unique isomorphism, and (i), (ii), (iii) together insure that $\gamma$ is a prefibration with transition functors $f^{*} \cong v^{*} \circ c^{*}$. Moreover, the second map in (1.3) is an isomorphism if $f \in C, g \in C$, or $f \in C, g \in V$, or $f \in V, g \in V$. It then remains to check that it is an isomorphism when $f \in V$ and $g \in C$, and this immediately follows from (iv).

1.5 Examples. Let us now give some general examples of fibrations and cofibrations in the sense of Definition 1.5.

Example 1.12. For any category $I$, the arrow category $\operatorname{ar}(I)=\operatorname{Fun}([1], I)$ is the category of arrows $i \rightarrow i^{\prime}$ in $I$, with morphisms from $f_{0}: i_{0} \rightarrow i_{0}^{\prime}$ to $f_{1}: i_{1} \rightarrow i_{1}^{\prime}$ given by commutative squares

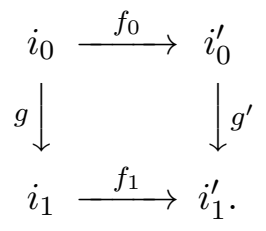

Consider the functors $s, t: \operatorname{ar}(I) \rightarrow I$ sending an arrow to its source resp. its target. Then $s$ is a fibration, its fibers $\operatorname{ar}(I)_{i}$ are the right-comma fibers $i \backslash^{\mathrm{ld}} I$ of Remark [1.6 of the identity functor Id $: I \rightarrow I$, and and a morphism $f$ is cartesian with respect to $s$ if and only if $t(f)$ is invertible. Dually, $t$ is a cofibration with fibers $\operatorname{ar}(I)_{i} \cong I /{ }^{\text {ld }} i$, and $f$ is cocartesian if and only if $s(f)$ is invertible.

Example 1.13. For any class of morphisms $V$ in $I$, let $\operatorname{ar}^{V}(I) \subset \operatorname{ar}(I)$ be the full subcategory spanned by arrows in $V$. Say that $V$ is closed under pullbacks if for any $f_{1}: i_{1} \rightarrow i_{1}^{\prime}$ in $V$ and any map $g^{\prime}: i_{0}^{\prime} \rightarrow i_{1}^{\prime}$, there exists a cartesian square (1.9) in $I$ with $f_{0} \in V$. Then in this case, the projection $t: \operatorname{ar}^{V}(I) \rightarrow I$ of Example 1.12 is a fibration, with cartesian maps corresponding to cartesian squares (1.9).

Example 1.14. For any category $I$, the fibration $t^{\perp}: \operatorname{tw}(I)=\operatorname{ar}(I)^{\perp} \rightarrow I^{o}$ transpose to the cofibration $t: \operatorname{ar}(I) \rightarrow I$ is the twisted arrow category of the 
category $I$ : its objects are arrows $f: i \rightarrow i^{\prime}$, and morphisms from $f_{0}: i_{0} \rightarrow i_{0}^{\prime}$ to $f_{1}: i_{1} \rightarrow i_{1}^{\prime}$ are given by commutative diagrams

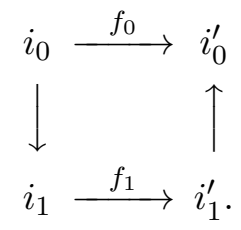

Note that the projection $s \times t^{\perp}: \operatorname{tw}(I) \rightarrow I \times I^{o}$ is also a fibration; it corresponds to the functor $I(-,-): I^{o} \times I=\left(I \times I^{o}\right)^{o} \rightarrow$ Sets $\subset$ Cat by the Grothendieck construction.

Example 1.15. By definition (see e.g. [Bou]), a factorization system $\langle L, R\rangle$ on a category $I$ consists of two closed classes of maps $L, R$ in $I$ such that $L \cap R=$ Iso, and any morphism $f: i \rightarrow i^{\prime}$ factors as

$$
i \stackrel{l}{\longrightarrow} \widetilde{i} \stackrel{r}{\longrightarrow} i^{\prime},
$$

with $l \in L, r \in R$, uniquely up to a unique isomorphism. Then for any factorization system $\langle L, R\rangle$, the projection $s: \operatorname{ar}^{L}(I) \rightarrow I$ of Example 1.12 is a fibration, and a map $f$ is cartesian iff $t(f) \in R$. Dually, $t: \operatorname{ar}^{R}(I) \rightarrow I$ is a cofibration, and a map $f$ is cocartesian iff $s(f) \in L$.

Moreover, if we are given a fibration $\pi: I^{\prime} \rightarrow I$, then the factorization system $\langle L, R\rangle$ lifts to a factorization system $\left\langle L^{\prime}, R^{\prime}\right\rangle$ on $I^{\prime}$, with $f \in R^{\prime}$ iff $\pi(f) \in R$ and $f$ is cartesian over $I$, and $f \in L^{\prime}$ iff $\pi(f) \in L$. In this case, we have $\operatorname{ar}^{R^{\prime}}\left(I^{\prime}\right) \cong \pi^{*} \operatorname{ar}^{R}(I)$.

While Example 1.7 is the protopytical example of a cofibration, it is Example 1.12. Example 1.13 and especially Example 1.15 that are very useful for actually constructing fibrations and cofibrations. For another useful general construction, consider a functor $\gamma: I^{\prime} \rightarrow I$ between small categories, and its left comma-fibers $I^{\prime} /{ }^{\gamma} i, i \in I$ of Remark 1.6. Then for any object $i \in I$, we have the forgetful functor $p_{i}: I^{\prime} / \gamma i I^{\prime},\langle c, \alpha\rangle \mapsto c$, and a map $f: i \rightarrow i^{\prime}$ induces a functor $f !: I^{\prime} /{ }^{\gamma} i \rightarrow I^{\prime} /{ }^{\gamma} i^{\prime},\langle c, \alpha\rangle \mapsto\langle c, f \circ \alpha\rangle$. For any fibration $\mathcal{C}^{\prime} \rightarrow I^{\prime}$, we can define a fibration $\gamma_{*} \mathcal{C}^{\prime} \rightarrow I$ with fibers

$$
\left(\gamma_{*} \mathcal{C}^{\prime}\right)_{i}=\operatorname{Sec}^{\natural}\left(I^{\prime} /{ }^{\gamma} i, p_{i}^{*} \mathcal{C}^{\prime}\right)
$$

and transition functors $\left(f_{!}\right)^{*}$. We then have a tautological evaluation functor $\gamma^{*} \gamma_{*} \mathcal{C}^{\prime} \rightarrow \mathcal{C}^{\prime}$, cartesian over $I^{\prime}$, and for any fibration $\mathcal{C} \rightarrow I$, we have a 
tautological functor $\mathcal{C} \rightarrow \gamma_{*} \gamma^{*} \mathcal{C}$, cartesian over $I$. This defines a sort of a 2 categorical adjunction between $\gamma^{*}$ and $\gamma_{*}$, in that for any fibrations $\mathcal{C}^{\prime} \rightarrow I^{\prime}$, $\mathcal{C} \rightarrow I$, we have a natural equivalence of categories

$$
\operatorname{Fun}_{I^{\prime}}^{\natural}\left(\gamma^{*} \mathcal{C}, \mathcal{C}^{\prime}\right) \cong \operatorname{Fun}_{I}^{\natural}\left(\mathcal{C}, \gamma_{*} \mathcal{C}^{\prime}\right)
$$

If $\gamma$ is a cofibration, one can use the equivalences (1.7) to replace the left comma-fibers $I^{\prime} /{ }^{\gamma} i$ in (1.11) with the usual fibers $I_{i}^{\prime}$. In fact, in this case, one can define a fibration $\gamma_{* *} \mathcal{C}^{\prime}$ with fibers $\left(\gamma_{* *} \mathcal{C}^{\prime}\right)_{i} \cong \operatorname{Sec}\left(I_{i}^{\prime}, \mathcal{C}_{i}^{\prime}\right)$, where $\mathcal{C}_{i}^{\prime}$ is the restriction of the fibration $\mathcal{C}^{\prime}$ to the fiber $I_{i}^{\prime} \subset I$, and transition functors

$$
\operatorname{Sec}\left(I_{i^{\prime}}^{\prime}, \mathcal{C}_{i^{\prime}}^{\prime}\right) \stackrel{\left(f_{!}\right)^{*}}{\longrightarrow} \operatorname{Sec}\left(I_{i}^{\prime},\left(f_{!}\right)^{*} \mathcal{C}_{i^{\prime}}^{\prime}\right) \longrightarrow \operatorname{Sec}\left(I_{i}^{\prime}, \mathcal{C}_{i}^{\prime}\right)
$$

for any map $f: i \rightarrow i^{\prime}$, where $f_{!}: I_{i}^{\prime} \rightarrow I_{i^{\prime}}^{\prime}$ is the transition functor of the cofibration $\gamma$, and the second functor is induced by the transition functors of the fibration $\mathcal{C}^{\prime} \rightarrow I^{\prime}$ along cocartesian liftings of the map $f$. Then $\gamma_{*} \mathcal{C}^{\prime} \subset \gamma_{* *} \mathcal{C}^{\prime}$ is the full subcategory spanned by $\operatorname{Sec}^{\natural}\left(I_{i}^{\prime}, \mathcal{C}_{i}^{\prime}\right) \subset \operatorname{Sec}\left(I_{i}^{\prime}, \mathcal{C}_{i}^{\prime}\right)$.

Remark 1.16. We note that the 2 -functor $I^{o} \rightarrow \mathcal{C}$ at corresponding to a fibration $\gamma_{*} \mathcal{C}^{\prime}$ of the form (1.11) is actually strict. This can be used for rigidifying arbitrary pseudofunctors: for any fibration $\mathcal{C} \rightarrow I$, we have $\mathcal{C} \cong \operatorname{ld}_{*} \mathcal{C}$, and $\operatorname{Id}_{*} \mathcal{C}$ corresponds to a strict 2 -functor $I^{o} \rightarrow \mathcal{C}$ at (in particular, we have a genuine functor $I^{o} \rightarrow$ Cat). In effect, one can consider the universal fibration $\mathrm{Cat}^{\bullet} \rightarrow \mathrm{Cat}^{\circ}$ corresponding to the tautological pseudofunctor Cat $\rightarrow \mathcal{C}$ at. Its fiber Cat ${ }_{\mathcal{C}}$ over some $\mathcal{C} \in$ Cat is $\mathcal{C}$ itself, and for any fibration $\mathcal{C} \rightarrow I$, there exists a functor $\gamma: I \rightarrow \mathrm{Cat}^{\circ}$ and an equivalence $\varepsilon: \mathcal{C} \cong \gamma^{*} \mathrm{Cat}^{\circ}$, and the pair $\langle\gamma, \varepsilon\rangle$ is unique up to a unique equivalence. However, these are equivalences and not isomorphisms - there is no way to state the Grothendieck construction purely in terms of the category Cat without introducing the notion of an equivalence. Replacing Cat with its quotient $\overline{\text { Cat }}$ of Lemma 1.1 does not help either, since a functor $I \rightarrow \overline{\text { Cat }}$ contains strictly less information that a pseudofunctor $I \rightarrow \mathcal{C}$ at (even if the latter is considered "up to an equivalence").

Example 1.17. Fix a Noetherian commutative ring $k$, denote by $\operatorname{Comm}(k)$ the category of finitely generated unital associative commutative $k$-algebras, and let $\operatorname{Comm}(k)$-mod be the category of pairs $\langle A, M\rangle, A \in \operatorname{Comm}(k)$, $M \in A$-mod an $A$-module, with maps $\langle A, M\rangle \rightarrow\left\langle A^{\prime}, M^{\prime}\right\rangle$ given by pairs $\langle f, g\rangle$ of an algebra map $f: A \rightarrow A^{\prime}$ and an abelian group map $g$ : $M \rightarrow M^{\prime}$ such that $g(a m)=f(a) g(m), a \in A, m \in M$. Then the obvious forgetful functor $\operatorname{Comm}(k)$-mod $\rightarrow \operatorname{Comm}(k)$ is bifibration, with fibers 
$\operatorname{Comm}(k)-\bmod _{A} \cong A$-mod, cofibration transition functors $M \mapsto A^{\prime} \otimes_{A} M$ and the tautological fibration transition functors sending an $A^{\prime}$-module $M$ to itself with the induced $A$-module structure.

More generally, let $\operatorname{Sch}(k)$ be the category of separated schemes of finite type over Spec $k$, let $j: \operatorname{Comm}(k)^{o} \rightarrow \operatorname{Sch}(k)$ be the embedding $A \mapsto \operatorname{Spec} A$, and denote $\mathrm{QCoh}(\operatorname{Sch}(k))=\left(j_{*} \operatorname{Comm}(k)-\bmod ^{o}\right)^{o}$. Then the cofibration $\mathrm{QCoh}(\operatorname{Sch}(k)) \rightarrow \operatorname{Sch}(k)^{o}$ is again a bifibration, and for any $X \in \operatorname{Sch}(k)$, the fiber $\mathrm{QCoh}(\operatorname{Sch}(k))_{X} \cong \mathrm{QCoh}(X)$ is the category of quasicoherent sheaves on $X$. Explicitly, objects in $\mathrm{QCoh}(\mathrm{Sch}(k))$ are pairs $\langle X, \mathcal{F}\rangle, X \in \mathrm{Sch}(k)$, $\mathcal{F} \in \mathrm{QCoh}(X)$, and maps $\left\langle X^{\prime}, \mathcal{F}^{\prime}\right\rangle \rightarrow\langle X, \mathcal{F}\rangle$ are pairs of a scheme map $f: X \rightarrow X^{\prime}$ and a sheaf map $f^{*} \mathcal{F} \rightarrow \mathcal{F}^{\prime}$ (note the change of variance). A scheme map $f$ defines a map $f^{o}$ in $\operatorname{Sch}(k)^{o}$, and the transition functors for the bifibration $\mathrm{QCoh}(\operatorname{Sch}(k)) \rightarrow \operatorname{Sch}(k)^{o}$ are $f^{o *} \cong f_{*}$ and $f_{!}^{o} \cong f^{*}$.

Even more generally, we can consider complexes of quasicoherent sheaves instead of sheaves themselves, denote by QCoh. $(\operatorname{Sch}(k)) \rightarrow \operatorname{Sch}(k)^{o}$ the corresponding bifibration, and restrict our attention to the full subcategory $\mathrm{QCoh}^{\mathrm{b}}(\mathrm{S} \operatorname{Sch}(k)) \subset$ QCoh. $(\mathrm{Sch}(k))$ of $h$-flat complexes (that is, complexes $\mathcal{F}$. such that $\mathcal{F}$. $\otimes_{\mathcal{O}_{X}}-$ preserves quasiisomorphisms). Since these are preserved by pullback functors $f^{*}$, the projection $\mathrm{QCoh}^{\mathrm{b}}(\mathrm{S} \operatorname{Sh}(k)) \rightarrow \operatorname{Sch}(k)^{o}$ is also a cofibration, and since pullbacks preserve quasiisomorphisms of $h$ flat complexes, we can then invert quasiisomorphisms in each fiber and obtain a cofibration $\mathcal{D}(\operatorname{Sch}(k)) \rightarrow \operatorname{Sch}(k)^{o}$ with fibers $\mathcal{D}(\operatorname{Sch}(k))_{X} \cong \mathcal{D}(X)$, the derived categories of $\mathrm{QCoh}(X)$. Its transition functors are $f_{!}^{o} \cong f^{*}$, and the isomorphisms (1.3) are induced by the corresponding isomorphisms for the cofibration QCoh $\mathrm{Q}^{\mathrm{b}}(\mathrm{S} \operatorname{ch}(k)) \rightarrow \mathrm{Sch}(k)^{o}$. This "derived" cofibration $\mathcal{D}(\operatorname{Sch}(k)) \rightarrow \operatorname{Sch}(k)^{o}$ is then again a bifibration, with fibration transition functors given by the derived functors $R^{\bullet} f_{*}$. Alternatively, one can start with $h$-injective complexes of flasque quasicoherent sheaves, and functors $f^{o *} \cong f_{*} ;$ the result is the same.

\section{Definitions and statements.}

2.1 Nerves and 2-categories. In practical applications, even if one considers 2-functors and not strict 2-functors, the notion of a strict 2-category is still too strict. One wants to relax it by allowing composition functors that are unital and associative only up to a fixed isomorphism, subject to higher constraints, and then one has to modify the notion of a 2-functor to take account of these extra pieces of data. A quick look at Definition 1.2 will show that this is maybe not the best of ideas (at least if one is intent on writ- 
ing down complete proofs). We will now describe an alternative formalism based of the notion of a "nerve".

As usual, we denote by $\Delta$ the category of ordinals $[n]=\{0, \ldots, n\}, n \geq 0$, and order-preserving maps between them. Since ordinals can be thought of as small categories, we have a fully faithful embedding $\delta: \Delta \subset$ Cat. We have $[n]^{o} \cong[n]$, and this defines an involution $\iota: \Delta \rightarrow \Delta$. For any $n \geq l \geq 0$, we denote by $s, t:[l] \rightarrow[n]$ the embeddings identifying $[l]$ with an initial resp. terminal segment of the ordinal $[n]$, and we note that we have a cocartesian square

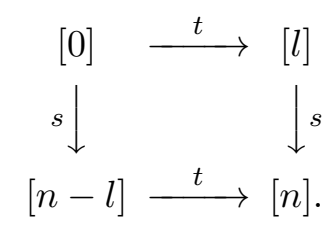

Recall that a simplicial set is a functor $X: \Delta^{o} \rightarrow$ Sets, and the nerve $N(I)$ of a small category $I$ is a simplicial set sending $[n]$ to the set of functors $[n] \rightarrow I$. In particular, 0-simplicies $i \in N(I)([0])$ are objects $i \in I$, and 1-simplices $f \in N(I)([1])$ are arrows $f: i \rightarrow i^{\prime}$. The two projections $N(I)(s), N(I)(t)$ : $N(I)([1]) \rightarrow N(I)([0])$ send an arrow to its source resp. its target. A small category is completely defined by its nerve, and a simplicial set $X$ is the nerve of a small category iff it sends cocartesian squares (2.1) to cartesian squares of sets (this is known as the Segal condition). One can also apply the Grothendieck construction to the nerve; this gives a fibration $\Delta I \rightarrow \Delta$ with discrete fibers $(\Delta I)_{[n]}=N(I)([n])$. The category $\Delta I$ is called the simplicial replacement of the category $I$.

Example 2.1. If one considers $[n] \in \Delta$ as a small category, then $\Delta[n]$ is the left comma-fiber $\Delta /^{\mathrm{ld}}[n]$ of Remark 1.6 of the identity functor - that is, the category of objects $[m] \in \Delta$ equipped with a map $[m] \rightarrow[n]$. In particular, we have the tautological object id $\in(\Delta[n])_{[n]} \subset \Delta[n]$ that corresponds to the identity map id : $[n] \rightarrow[n]$.

We now observe that the correspondence $I \mapsto \Delta I$ can be immediately generalized to strict 2-categories.

Definition 2.2. The simplicial replacement $\Delta \mathcal{C}$ of a strict 2-category $\mathcal{C}$ is the fibration $\Delta \mathcal{C} \rightarrow \Delta$ with fibers $(\Delta \mathcal{C})_{[n]}=\operatorname{Fun}^{2}([n], \mathcal{C})$, and transition functors given by pullbacks with respect to maps $f:[n] \rightarrow[m]$.

By Definition 1.3 , if $\mathcal{C}=I$ is a usual category, then $\operatorname{Fun}^{2}([n], I)$ is exactly the set $N(I)([n])$, so that in this case, Definition 2.2 reduces to our previous 
notion of a simplicial replacement. We also note that any 2-functor $\gamma: \mathcal{C} \rightarrow$ $\mathcal{C}^{\prime}$ induces a functor $\Delta(\gamma): \Delta \mathcal{C} \rightarrow \Delta \mathcal{C}^{\prime}$, cartesian over $\Delta$, and as soon as $\mathcal{C}$ is small, we also have a functor

$$
\operatorname{Fun}^{2}\left(\mathcal{C}, \mathcal{C}^{\prime}\right) \rightarrow \operatorname{Fun}_{\Delta}^{\natural}\left(\Delta \mathcal{C}, \Delta \mathcal{C}^{\prime}\right) .
$$

For any $\mathcal{C}$, the fiber $(\Delta \mathcal{C})_{[0]}$ is discrete and consists of objects of $\mathcal{C}$, while $(\Delta \mathcal{C})_{[1]}$ is the disjoint union of all the categories of morphisms $\mathcal{C}(-,-)$. Moreover, say that a fibration $\mathcal{E} \rightarrow \Delta$ satisfies the Segal condition if for any square (2.1), the corresponding functor

$$
s^{*} \times t^{*}: \mathcal{E}_{[n]} \rightarrow \mathcal{E}_{[l]} \times \mathcal{E}_{[0]} \mathcal{E}_{[n-l]}
$$

is an equivalence of categories. Then $\Delta \mathcal{C}$ always satisfies the Segal condition. In particular, for $n=2, l=1$, the category $(\Delta \mathcal{C})_{[2]} \cong(\Delta \mathcal{C})_{[1]} \times{ }_{(\Delta \mathcal{C})_{[0]}}(\Delta \mathcal{C})_{[1]}$ is the category of composable pairs of morphisms in $\mathcal{C}$. The composition is then given by the transition functor

$$
m^{*}:(\Delta \mathcal{C})_{[2]} \cong(\Delta \mathcal{C})_{[1]} \times{ }_{(\Delta \mathcal{C})_{[0]}}(\Delta \mathcal{C})_{[1]} \rightarrow(\Delta \mathcal{C})_{[1]}
$$

corresponding to the embedding $m:[1] \rightarrow[2], 0 \mapsto 0,1 \mapsto 2$, and the identity morphisms are given by the embedding

$$
e^{*}:(\Delta \mathcal{C})_{[0]} \rightarrow(\Delta \mathcal{C})_{[1]}
$$

corresponding to the unique projection $e:[1] \rightarrow[0]$.

Lemma 2.3. For any strict 2-categories $\mathcal{C}, \mathcal{C}^{\prime}$ with $\mathcal{C}$ small, the functor (2.2) is an equivalence.

Proof. To obtain an inverse equivalence, note that a functor $\gamma: \Delta \mathcal{C} \rightarrow \Delta \mathcal{C}^{\prime}$ over $\Delta$ induces a map of objects $\gamma([0])$, and a functor $\gamma([1])$ of the categories of morphisms. If $\gamma$ is cartesian, then we also have the isomorphisms (1.5) for the maps $m, e$ of (2.4), (2.5), and these provide the functorial isomorphisms of Definition 1.2. A map $\alpha: \gamma \rightarrow \gamma^{\prime}$ between two cartesian functors has the component $\alpha([0])$ that must be an identity map since $\left(\Delta \mathcal{C}^{\prime}\right)_{[0]}$ is discrete, and $\alpha([1])$ gives a 2-morphism in the sense of Definition 1.3 .

Corollary 2.4. A 2-functor $\gamma$ is a strong equivalence in the sense of Definition 1.4 if and only if $\Delta(f)$ is an equivalence of categories.

Proof. Clear. 
Definition 2.5. A 2-category is a fibration $\mathcal{C} \rightarrow \Delta$ satisfying the Segal condition (2.3) such that the fiber $\mathcal{C}_{[0]}$ is discrete. A 2-functor between 2-categories $\mathcal{C}, \mathcal{C}^{\prime}$ is a functor $\gamma: \mathcal{C} \rightarrow \mathcal{C}^{\prime}$ cartesian over $\Delta$.

Motivated by Lemma 2.3, we denote $\operatorname{Fun}^{2}\left(\mathcal{C}, \mathcal{C}^{\prime}\right)=\operatorname{Fun}_{\Delta}^{\natural}\left(\mathcal{C}, \mathcal{C}^{\prime}\right)$ for any 2-categories $\mathcal{C}, \mathcal{C}^{\prime}$ in the sense of Definition 2.5, and we let $\operatorname{Fun}^{2}(I, \mathcal{C})=$ $\operatorname{Fun}^{2}(\Delta I, \mathcal{C})$ for a small category $I$. We note that by virtue of Example 2.1, we have a tautological equivalence $\mathrm{Y}: \operatorname{Fun}^{2}([n], \mathcal{C}) \cong \mathcal{C}_{[n]}$ sending a 2-functor $\gamma$ to its value on id $\in(\Delta[n])_{[n]}$. For any strict 2-category $\mathcal{C}$, the simplicial replacement $\Delta \mathcal{C}$ is a 2-category in the sense of Definition 2.5, and for any 2-functor $\gamma: \mathcal{C} \rightarrow \mathcal{C}^{\prime}$, the functor $\Delta(\gamma): \Delta \mathcal{C} \rightarrow \Delta \mathcal{C}^{\prime}$ is a 2-functor. An arbitrary 2-category $\mathcal{C}$ in the sense of Definition 2.5 still has objects $c \in \mathcal{C}_{[0]}$, and just as in the strict case, the projection $s^{*} \times t^{*}: \mathcal{C}_{[1]} \rightarrow \mathcal{C}_{[0]}$ provides a decompositon

$$
\mathcal{C}_{[1]}=\coprod_{c, c^{\prime} \in \mathcal{C}_{[0]}} \mathcal{C}\left(c, c^{\prime}\right)
$$

into categories of morphisms. Then (2.4) and (2.5) provide composition functors and identity objects. However, in general, all the axioms only hold up to canonical isomorphisms induced by the maps (1.5) for the structural fibration $\mathcal{C} \rightarrow \Delta$.

For any 2-category $\mathcal{C}$, the 2-opposite category is $\mathcal{C}^{\tau}=\mathcal{C}_{\perp}^{o}$, and the 1 opposite 2-category is $\mathcal{C}^{\iota}=\iota^{*} \mathcal{C}$, where $\iota: \Delta \rightarrow \Delta$ is the involution $[n] \mapsto[n]^{o}$. The 2-product of 2-categories $\mathcal{C}, \mathcal{C}^{\prime}$ is given by $\mathcal{C} \times{ }^{2} \mathcal{C}^{\prime}=\mathcal{C} \times{ }_{\Delta} \mathcal{C}^{\prime}$. Note that any object $c \in \mathcal{C}_{[0]}^{\prime}$ uniquely extends to a cartesian section $\iota(c): \Delta \rightarrow \mathcal{C}^{\prime}$ of the fibration $\mathcal{C}^{\prime} \rightarrow \Delta$, and this gives a full embedding id $\times \iota(c): \mathcal{C} \rightarrow \mathcal{C} \times{ }^{2} \mathcal{C}^{\prime}$ whose essential image is denoted by $\mathcal{C} \times\{c\} \subset \mathcal{C}^{\prime}$.

Remark 2.6. Definition [2.5] is essentially the same as [K3, Definition 6.1] but there is one difference: $\mathrm{K} 3$ ] describes a 2-category $\mathcal{C}$ of Definition 2.5 in terms of the transpose cofibration $\mathcal{C}_{\perp} \rightarrow \Delta^{o}$ rather then the fibration $\mathcal{C} \rightarrow \Delta$. The two approaches are equivalent but one has to choose one. It seems that fibrations make for simpler formulas when dealing with sets and geometric objects, while cofibrations work better for rings, algebras and the like. Since [K3] deals with algebra rather than geometry, cofibrations were chosen there, and this is the choice that would have been better for Section 4 below. However, for the rest of this Section and for Section 3 fibrations are more convenient, so this is what we use. 
2.2 Grothendieck construction for 2-categories. As a first example for Definition 2.2, let us describe the simplicial replacement $\Delta \mathcal{C}$ at of the 2-category $\mathcal{C}$ at. We first observe that any fibration $\mathcal{E} \rightarrow \Delta$ that satisfies the Segal condition can be turned into a 2-category in the following way. Take any fibration $\mathcal{E} \rightarrow \Delta$, let $\varepsilon:$ pt $\rightarrow \Delta$ be the embedding onto [0] $\in \Delta$, let $\mathcal{E}_{0}=\mathcal{E}_{[0]}=\varepsilon^{*} \mathcal{E}$, and let $\mathcal{E}_{0}^{\prime}=\mathcal{E}_{0, \text { Id }} \subset \mathcal{E}_{0}$ be its underlying discrete subcategory. Define the reduction $\mathcal{E}^{\text {red }}$ of the fibration $\mathcal{E}$ by the cartesian square

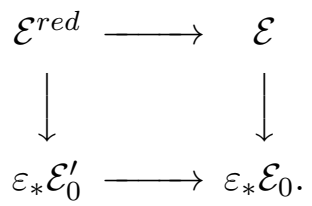

Then the square is fibered over $\Delta$, and by (1.11), we have $\left(\varepsilon_{*} \mathcal{E}_{0}\right)_{[n]} \cong \mathcal{E}_{0}^{n+1}$ for any $[n] \in \Delta$, and similarly for $\mathcal{E}_{0}^{\prime}$. Thus both categories in the bottom line of (2.7) satisfy the Segal condition. Then if so does $\mathcal{E}$, the same holds for $\mathcal{E}^{\text {red }}$, and since $\mathcal{E}_{[0]}^{\text {red }}=\mathcal{E}_{0}^{\prime}$ is discrete, it is a 2-category in the sense of Definition 2.5.

Now let $c$ be the class of all cofibrations in Cat, consider the fibration $t: \operatorname{ar}^{c}$ (Cat) $\rightarrow$ Cat of Example 1.13 (that applies by Remark 1.9), and let $\mathcal{E}=\delta^{*} \operatorname{ar}^{c}$ (Cat) be its restriction with respect to the full embedding $\delta: \Delta \rightarrow$ Cat. We claim that

$$
\Delta \mathcal{C} a t \cong \mathcal{E}^{r e d}
$$

Indeed, by definition, for any $[n] \in \Delta, \mathcal{E}_{[n]} \cong \operatorname{Cat}_{\natural} /[n]$ is the category of small categories cofibered over $[n]$, and strict cocartesian functors between them. By the Grothendieck construction, these categories correspond to 2-functors $[n] \rightarrow \mathcal{C}$ at. Morphisms between some $\mathcal{C}, \mathcal{C}^{\prime} \in \mathcal{E}_{[n]}$ are strict cocartesian functors $\gamma: \mathcal{C} \rightarrow \mathcal{C}^{\prime}$ over $[n]$, but morphisms in the reduction $\mathcal{E}_{[n]}^{\text {red }}$ are functors such that $\gamma(l)=$ id for any $l \in[n]$. The only remaining data are then the morphisms (1.5) - or rather, their cofibration analogs - and these correspond on the nose to 2-morphisms of Definition 1.3.

Remark 2.7. The reader might wonder what changes if instead of strict cocartesian functors we consider all cocartesian functors. The answer is "in the end, nothing": while $\mathcal{E}$ would change, its reduction would be the same.

As an application of the identification (2.8), let us describe a version the Grothendieck construction of Subsection 1.3 with $I$ replaced by an arbitrary 
2-category $\mathcal{C}$. Say that a map $f:[m] \rightarrow[n]$ in $\Delta$ is special if $f(0)=0$. Denote by + the class of all special maps, with $\Delta_{+} \subset \Delta$ being the corresponding dense subcategory, and let $t$ be the class of all terminal embeddings $t:[m] \rightarrow$ $[n]$. Then $\langle+, t\rangle$ is a factorization system on $\Delta$, and for any 2-category $\mathcal{C}$, we have its lifting $\langle+, t\rangle$ of Example 1.15. Say that a map $f$ in $\mathcal{C}$ is special if $f \in+$ (that is, $f$ goes to a special map under the fibration $\mathcal{C} \rightarrow \Delta$ ).

Definition 2.8. A fibration $\mathcal{C}^{\prime} \rightarrow \mathcal{C}$ over a 2-category $\mathcal{C}$ is special if for any special map $f$ in $\mathcal{C}$, the transition functor $f^{*}$ is an equivalence.

In the prototypical example of a special fibration, $\mathcal{C}$ is $\Delta \mathcal{C}$ at, and the fibration $\Delta^{*} \mathcal{C}$ at $\rightarrow \Delta \mathcal{C}$ at has fibers

$$
\Delta^{\bullet} \mathcal{C} a t_{\mathcal{C}}=\operatorname{Sec}_{\natural}([n], \mathcal{C}),
$$

where the cofibration $\mathcal{C} \rightarrow[n]$ corresponds to an object in $\Delta \mathcal{C}$ at via (2.8). The transition functors are given by pullbacks. Since $0 \in[n]$ is the initial object, we have the equivalence (1.7) - or rather, its analog for cofibrations and cocartesian sections - and in our case, it reads as $\operatorname{Sec}_{\curvearrowleft}([n], \mathcal{C}) \cong \mathcal{C}_{0}$. Therefore the fibration (2.9) is special. It is also universal, in the following sense.

Lemma 2.9. For any special fibration $\mathcal{C}^{\prime} \rightarrow \mathcal{C}$ over a 2-category $\mathcal{C}$, there exists a 2-functor $\gamma: \mathcal{C} \rightarrow \Delta \mathcal{C}$ at such that $\mathcal{C}^{\prime} \cong \gamma^{*} \Delta^{*} \mathcal{C}$ at.

Proof. Note that for any $[n] \in \Delta$, the fiber $\operatorname{ar}^{t}(\Delta)_{[n]}$ of the cofibration $t$ of Example 1.15 is naturally identified with $[n]^{o}$, by sending $t:[m] \rightarrow[n]$ to $t(0) \in[n]$, and the same is then true for the fibers of the cofibration $t: \operatorname{ar}^{t}(\mathcal{C}) \rightarrow \mathcal{C}$. For any $c \in \mathcal{C}$, the projection $s: \operatorname{ar}^{t}(\mathcal{C}) \rightarrow \mathcal{C}$ restricts to a functor $s_{c}:[n]^{o} \cong \operatorname{ar}^{t}(\mathcal{C})_{c} \rightarrow \mathcal{C}$, so we have the fibration $s_{c}^{*} \mathcal{C}^{\prime} \rightarrow[n]^{o}$. The functor $\gamma$ sends $c$ to the transpose cofibration $\left(s_{c}^{*} \mathcal{C}^{\prime}\right)_{\perp} \rightarrow[n]$.

Remark 2.10. The fibration $t^{o}: \operatorname{ar}^{t}(\Delta)^{o} \rightarrow \Delta^{o}$ opposite to the cofibration $t$ corresponds to the tautological embedding $\Delta \subset$ Cat by the Grothendieck construction. Another interpretation is that $\operatorname{ar}^{t}(\Delta)$ is the category $\Delta^{*}$ of pairs $\langle[n], l\rangle,[n] \in \Delta, l \in[n]$, with maps from $\langle[n], l\rangle$ to $\left\langle\left[n^{\prime}\right], l^{\prime}\right\rangle$ given by maps $f:[n] \rightarrow\left[n^{\prime}\right]$ such that $f(l) \geq l^{\prime}$. Then $t$ is the forgetful functor $\langle[n], l\rangle \mapsto[n]$, and $s: \operatorname{ar}^{t}(\Delta) \rightarrow \Delta$ sends $\langle[n], l\rangle$ to $[n-l]$. This interpretation is quite useful in many constructions involving nerves. For example, the simplicial replacement $\Delta I$ of a category $I$ is given by

$$
\Delta I=\left(t_{* *}\left(I \times \operatorname{ar}^{t}(\Delta)\right)\right)^{r e d},
$$


where $t_{* *}$ is as in (1.12), and $I \times \operatorname{ar}^{t}(\Delta) \rightarrow \operatorname{ar}^{t}(\Delta)$ is the constant fibration with fiber $I$.

Remark 2.11. Ideally, we would like to say that $\gamma$ in Lemma 2.9 is unique up to an isomorphism, but this is not true. The reason for this is the reduction procedure used in (2.8): by definition, objects in $(\Delta \mathcal{C} a t)_{[0]}$ are small categories on the nose, and two isomorphic but different categories stop being isomorphic as objects in $\Delta \mathcal{C}$ at. The real way to get rid of artefacts of this type would be to relax the condition of Definition 2.5 and allow $\mathcal{C}_{[0]}$ to be a groupoid; however, this would take one rather far away from the standard theory of 2-categories. As a temporary fix, one can choose a specific model for Cat and similar categories where there is only one object in each isomorphism class. Then $\gamma$ in Lemma 2.9 becomes unique up to a unique isomorphism.

If we fix a small category $I$, and fix a concrete model for Cat as in Remark 2.11, that the equivalence (1.6) extends to a strong equivalence

$$
\mathcal{C} a t^{\natural} / I \cong \mathcal{C} a t_{\natural} / I^{o}
$$

and all of the material in this Subsection immediately generalizes to these strongly equivalent strict 2-categories, with the same proofs. Namely, for any 2-category $\mathcal{C}$, a fibration $\mathcal{C}^{\prime} \rightarrow I \times \mathcal{C}$ is special if so is its composition with the projection $I \times \mathcal{C} \rightarrow \mathcal{C}$. Objects in the simplicial replacement $\Delta\left(\mathcal{C} a t_{\natural} / I^{o}\right)$ are pairs $\langle[n], \mathcal{C}\rangle$ of an ordinal $[n] \in \Delta$ and a small cofibration $\mathcal{C} \rightarrow I^{o} \times[n]$, and one defines a special fibration $\Delta^{*}\left(\mathcal{C} a t_{\natural} / I^{o}\right) \rightarrow I \times \Delta\left(\mathcal{C} a t_{\natural} / I^{o}\right)$ by setting $\Delta^{\bullet}\left({\mathcal{C} a t_{\natural}}_{\natural} / I^{o}\right)_{\mathcal{C}}=\pi_{*} \mathcal{C}^{\perp}$, where $\mathcal{C}^{\perp} \rightarrow I \times[n]^{o}$ is the transpose fibration to $\mathcal{C}$, and $\pi: I \times[n]^{o} \rightarrow I$ is the projection. Then exactly the same argument as in Lemma 2.9 shows that for any 2-category $\mathcal{C}$ equipped with a special fibration $\mathcal{C}^{\prime} \rightarrow I \times \mathcal{C}$, there exists a 2-functor $\gamma: \mathcal{C} \rightarrow \Delta\left(\mathcal{C} a t_{\natural} / I^{o}\right)$ and an equivalence $\mathcal{C}^{\prime} \cong(\gamma \times \mathrm{Id})^{*} \Delta^{\cdot}\left(\mathcal{C} a t_{\natural} / I^{o}\right)$, with the uniqueness properties described in Remark 2.11,

2.3 Rigidification. Let us now show that our Definition 2.5 is equivalent to the usual definition of weak 2-categories and 2-functors that uses associativity isomorphisms and suchlike. Fortunately, it has already been proved in $[\mathrm{Be}$ that any weak 2-category in the usual sense is equivalent to a strict one, so it suffices to identify 2-categories and 2-functors of Definition 2.5 with strict 2-categories and 2-functors. For 2-functors, this is Lemma 2.3, and the result for 2-categories is as follows. 
Theorem 2.12. For any small 2-category $\mathcal{C}$ in the sense of Definition 2.5, there exists a small strict 2 -category $\mathrm{R}(\mathcal{C})$ and an equivalence $\mathcal{C} \cong \Delta \mathrm{R}(\mathcal{C})$, and $\mathrm{R}(\mathcal{C})$ is unique up to a strong equivalence in the sense of Definition 1.4 .

The idea of the proof of Theorem 2.12 is to use the Yoneda embedding. We consider the 1-opposite 2-category $\mathcal{C}^{\iota}$, and to any object $c \in \mathcal{C}_{[0]}$, we associate a representable 2 -functor $\mathcal{C}^{\iota} \rightarrow \mathcal{C}$ at. We then apply the Grothendieck construction of Lemma 2.9 to encode these 2-functors by special fibrations $\mathcal{C}^{\iota}(c) \rightarrow \mathcal{C}^{\iota}$, we get a full embedding $\mathcal{C} \rightarrow \Delta\left(\mathcal{C} a t^{\natural} / \mathcal{C}^{\iota}\right)$, and $\mathcal{C} a t^{\natural} / \mathcal{C}^{\iota}$ is strict. In order to make this work, we first need a 2-categorical version of the Yoneda Lemma.

Let $\rho: \Delta_{+} \rightarrow \Delta$ be the embedding functor, and note that it has a leftadjoint $\lambda: \Delta \rightarrow \Delta_{+}$that adds a new initial element to an ordinal $[n]$. The composition $\kappa=\rho \circ \lambda: \Delta \rightarrow \Delta$ sends an ordinal $[n]$ to $[n]^{<} \cong[n+1]$ and comes equipped with the adjunction map $a:$ Id $\rightarrow \kappa$ (given by the terminal segment embeddings $t:[n] \rightarrow[n+1])$. By Remark 1.6, since [0] $\in \Delta_{+}$is the initial object, any fibration $\mathcal{E} \rightarrow \Delta_{+}$comes equipped with a functor $\mathcal{E} \rightarrow \mathcal{E}_{[0]}$ right-adjoint to the embedding $\mathcal{E}_{[0]} \rightarrow \mathcal{E}$. In particular, for any 2-category $\mathcal{C}$, we can take $\mathcal{C}_{+}=\rho^{*} \mathcal{C} \rightarrow \Delta_{+}$, and we obtain a decomposition

$$
\mathcal{C}_{+} \cong \coprod_{c \in \mathcal{C}_{[0]}} \mathcal{C}_{+}(c)
$$

Applying $\lambda^{*}$, we obtain a decomposition of the fibration $\kappa^{*} \mathcal{C} \cong \lambda^{*} \mathcal{C}_{+}$into components $\mathcal{C}(c)=\lambda^{*} \mathcal{C}_{+}(c), c \in \mathcal{C}_{[0]}$, and for any $c$, the transition functor $a^{*}$ provides a projection

$$
\pi=a^{*}: \mathcal{C}(c) \rightarrow \mathcal{C} .
$$

The Segal condition immediately shows that this is a fibration. Moreover, any object $c^{\prime} \in \mathcal{C}_{[n]} \subset \mathcal{C}$ defines an object $s^{*} c^{\prime} \in \mathcal{C}_{[0]}$, where $s:[0] \rightarrow[n]$ is the initial embedding, and again by the Segal condition, the fibers of the fibration (2.11) are given by

$$
\mathcal{C}(c)_{c^{\prime}} \cong \mathcal{C}\left(c, s^{*} c^{\prime}\right),
$$

where $\mathcal{C}(-,-)$ are the components of the decomposition (2.6). In particular, the fibration (2.11) is special. Moreover, we have a distinguished object $\mathrm{id}_{c} \in \mathcal{C}(c, c) \cong \mathcal{C}(c)_{c} \subset \mathcal{C}(c)$.

Lemma 2.13. For any small 2-category $\mathcal{C}$ and $c, c^{\prime} \in \mathcal{C}_{[0]}$, the functor

$$
\mathrm{Y}: \operatorname{Fun}_{\mathcal{C}}^{\natural}\left(\mathcal{C}(c), \mathcal{C}\left(c^{\prime}\right)\right) \rightarrow \mathcal{C}\left(c^{\prime}, c\right), \quad \gamma \mapsto \gamma\left(\operatorname{id}_{c}\right)
$$

is an equivalence of categories. 
Proof. The embedding $\lambda_{c}: \mathcal{C}(c) \cong \lambda^{*} \mathcal{C}_{+}(c) \rightarrow \mathcal{C}_{+}(c)$ admits a right-adjoint functor $\rho_{c}: \mathcal{C}_{+}(c) \rightarrow \mathcal{C}(c)$ whose composition $\pi_{c}=\pi \circ \rho_{c}: \mathcal{C}_{+}(c) \rightarrow \mathcal{C}$ with the fibration $\pi$ of (2.11) is obtained by restricting the natural embedding $\rho: \mathcal{C}_{+}=\rho^{*} \mathcal{C} \rightarrow \mathcal{C}$ to the corresponding component of (2.10). For any map $f$ in $\mathcal{C}_{+}(c)$, the map $\rho_{c}(f)$ in $\mathcal{C}(c)$ is cartesian with respect to $\pi$, and for any object $x \in \mathcal{C}(c)$, so is the adjunction map $a_{c}(x): x \rightarrow \kappa_{c}(x)$, where $\kappa_{c}=\rho_{c} \circ \lambda_{c}$. Therefore for any functor $F: \mathcal{C}(c) \rightarrow \mathcal{C}\left(c^{\prime}\right)$ cartesian over $\mathcal{C}$, and any object $x \in \mathcal{C}(c)$, we have $F(x) \cong \pi\left(a_{c}(x)\right)^{*} F\left(\kappa_{c}(x)\right)$, and the isomorphism is functorial with respect to $F$ and $x$. This means that we have an equivalence

$$
\operatorname{Fun}_{\mathcal{C}}^{\natural}\left(\mathcal{C}(c), \mathcal{C}\left(c^{\prime}\right)\right) \cong \operatorname{Sec}^{\natural}\left(\mathcal{C}_{+}(c), \pi_{c}^{*} \mathcal{C}\left(c^{\prime}\right)\right), \quad F \mapsto \rho_{c}^{*} F,
$$

and it remains to observe that since $\pi_{c}: \mathcal{C}_{+}(c) \rightarrow \mathcal{C}$ sends all maps to special maps, and the fibration $\mathcal{C}\left(c^{\prime}\right) \rightarrow \mathcal{C}$ is special, all the transition functors of the fibration $\pi_{c}^{*} \mathcal{C}\left(c^{\prime}\right)$ are equivalences. Therefore it is a bifibration, its cartesian sections coincide with cocartesian ones, and by the cofibration verison of (1.7), the right-hand side of (2.14) is equivalent to the fiber $\mathcal{C}\left(c^{\prime}, c\right) \cong \pi_{c}^{*} \mathcal{C}\left(c^{\prime}\right)_{o}$ over the initial object $o \in \mathcal{C}_{+}(c)$.

Proof of Theorem 2.12. Uniqueness immediately follows from Corollary 2.4. To prove existence, say that the concatenation $[l] \circ[n]$ of any two ordinals $[l],[n] \in \Delta$ is their disjoint union $[l] \sqcup[n]$ ordered left-to-right, and denote by $m: \Delta^{2} \rightarrow \Delta$ the functor sending $[l] \times[n]$ to $[l] \circ[n]$. Moreover, let $p_{0}, p_{1}: \Delta^{2} \rightarrow \Delta$ be the projections onto the first and second factor, and note that we have natural maps $a_{0}: p_{0} \rightarrow m, a_{1}: p_{1} \rightarrow m$. Let $\mathcal{C}^{(2)}=m^{*} \mathcal{C}$, and note that the transition functors $a_{0}^{*}, a_{1}^{*}$ induce projections $\pi_{0}, \pi_{1}: \mathcal{C}^{(2)} \rightarrow \mathcal{C}$. Consider the product

$$
\pi=\left(\iota \circ \pi_{0}\right) \times \pi_{1}: \mathcal{C}^{(2)} \rightarrow \mathcal{C}^{\iota} \times \mathcal{C} .
$$

Then the Segal condition immediately shows that this is a fibration. Moreover, its composition $\mathcal{C}^{(2)} \rightarrow \mathcal{C}$ with the projection $\mathcal{C}^{\iota} \times \mathcal{C} \rightarrow \mathcal{C}$ is also a fibration with fibers $\mathcal{C}_{c}^{(2)} \cong \mathcal{C}^{\iota}\left(s^{*} c\right)$, where $s^{*} c$ is as in (2.12). Therefore the fibration (2.15) is special, and if we denote by $R(\mathcal{C}) \subset \mathcal{C} a t^{\natural} / \mathcal{C}^{\iota}$ the full 2-subcategory spanned by special fibrations $\mathcal{C}^{\iota}(c) \rightarrow \mathcal{C}^{\iota}, c \in \mathcal{C}_{[0]}^{\iota}=\mathcal{C}_{[0]}$, then Lemma 2.9 provides a 2 -functor

$$
\mathrm{Y}: \mathcal{C} \rightarrow \Delta \mathrm{R}(\mathcal{C})
$$

By construction, this functor is an identity over $[0] \in \Delta$, and for any objects $c, c^{\prime} \in \mathcal{C}_{[0]}$, its restriction $\mathrm{Y}\left(c, c^{\prime}\right)$ to the component $\mathcal{C}\left(c, c^{\prime}\right)$ of the decomposition (2.6) is inverse to the equivalence (2.13) of Lemma 2.13. Therefore 
$\mathrm{Y}\left(c, c^{\prime}\right)$ is also an equivalence, so that (2.16) is an equivalence over [1]. By the Segal condition, it is an equivalence.

Remark 2.14. The main reason we restrict our attention to small categories in Lemma 2.13 and Theorem 2.12 is to insure that everything is well-defined - a priori, there could be more than a set of morphisms between two functors between large categories. However, it is easy to see that even for a large $\mathcal{C}$, Lemma 2.13 works with the same proof, and a posteriori, functors from $\mathcal{C}(c)$ to $\mathcal{C}\left(c^{\prime}\right)$ cartesian over $\mathcal{C}$ form a well-defined category. The Yoneda embedding of Theorem 2.12 works for large 2-categories, too.

2.4 Monoidal structures. Let us now consider a special class of 2-categories - those that only have one object. These correspond to unital monoidal structures.

Definition 2.15. A unital monoidal structure on a category $\mathcal{C}$ is given by a 2-category $B \mathcal{C}$ with $B \mathcal{C}_{[0]} \cong \mathrm{pt}$, equipped with an equivalence $B \mathcal{C}_{[1]} \cong \mathcal{C}$. A unital monoidal structure on a functor $\gamma: \mathcal{C} \rightarrow \mathcal{C}^{\prime}$ between categories $\mathcal{C}$, $\mathcal{C}^{\prime}$ with unital monoidal structures $B \mathcal{C}, B \mathcal{C}^{\prime}$ is a 2-functor $B \gamma: B \mathcal{C} \rightarrow B \mathcal{C}^{\prime}$ that restricts to $\gamma$ over [1].

In this definition, 2-categories and 2-functors are understood in the sense of Definition 2.5. One can also consider strict unital monoidal categories by requiring $B C$ to be a strict 2-category, but this is not very useful in practical applications. Some very simple monoidal structures that exist in nature are indeed strict; one such is the concatenation product used in the proof of Theorem 2.12 (it has to be strict by necessity, since the only isomorphisms in $\Delta$ are the identity maps). However, even this simple example admits a more efficient description in terms of Definition 2.5. To construct the corresponding 2-category $B \Delta$, we need some simplicial combinatorics.

Recall from Subsection 2.2 that we denote by + the class of special maps in $\Delta$, and that the embedding $\rho: \Delta_{+} \rightarrow \Delta$ admits a left-adjoint $\lambda: \Delta \rightarrow \Delta_{+}$, $[n] \mapsto[n]^{<}$. Note that $\lambda$ extends to the category $\Delta^{<}$, where we interpret the new initial object $o \in \Delta^{<}$as the empty ordinal. Dually, say that a map $f:[l] \rightarrow[n]$ is antispecial if $\iota(f)$ is special (or equivalently, $f(l)=n$ ), let - be the class of antispecial maps, and note that $\iota: \Delta \rightarrow \Delta$ induces an equivalence $\Delta_{+} \cong \Delta_{-}$, while the embedding $\rho_{\iota}=\iota \circ \rho \circ \iota: \Delta_{-} \rightarrow \Delta$ admits a left-adjoint $\lambda_{\iota}=\iota \circ \lambda \circ \iota: \Delta \rightarrow \Delta_{+},[n] \mapsto[n]^{>}$that also extends to $\Delta^{<}$. Moreover, say that a map $f$ is bispecial if it is both special and antispecial, let $\pm=+\cap-$, and note that the embedding $\rho_{b}=\rho \circ \rho_{\iota}: \Delta_{ \pm} \rightarrow \Delta$ admits a 
left-adjoint $\lambda_{b}: \Delta \rightarrow \Delta_{ \pm}$that also extends to $\Delta^{<}$. Note that $[0] \in \Delta$ is both the initial and the terminal object both for $\Delta_{+}$and $\Delta_{-}$and the terminal object for $\Delta_{ \pm}$, while the initial object for $\Delta_{ \pm}$is [1].

Next, recall that we have the factorization system $\langle+, t\rangle$ on $\Delta$, where $t$ stands for the class of terminal embeddings $t:[l] \rightarrow[n]$, and dually, we have the factorization system $\langle-, s\rangle$, where $s$ is the class of the initial embeddings $s:[l] \rightarrow[n]$. Say that a map $f$ in $\Delta$ is an anchor map if it decomposes as $f=s \circ t$, with $s$ resp. $t$ an initial resp. a terminal embedding, or equivalently, $f:[m] \rightarrow[n]$ is injective and identifies the ordinal $[m]$ with some segment $\{l, l+1, \ldots, l+m\} \subset[n]$ of the ordinal $[n]$. Then we also have a factorization system $\langle \pm, a\rangle$ on $\Delta$, where $a$ stands for the class of anchor maps.

Finally, observe that if we treat a map $f:[m] \rightarrow[n]$ in $\Delta$ as a functor between small categories, then a right-adjoint $f^{\dagger}:[n] \rightarrow[m]$ is by definition given by $f^{\dagger}(l)=\max \left\{l^{\prime} \in[m] \mid f\left(l^{\prime}\right) \leq l\right\}$. This is well-defined if and only if all the sets in the right-hand side are non-empty, or equivalently, $f(0) \leq 0$, so that $f$ must be special. The adjoint $f^{\dagger}$ is then automatically anti-special, and we obtain equivalences

$$
\Delta_{+} \cong \Delta_{-}^{o}, \quad \Delta_{+}^{o} \cong \Delta_{-} .
$$

Moreover, $f^{\dagger}$ is special if and only if $f^{-1}(0)=0$, and this means that $f$ lies in the image of the functor $\lambda: \Delta^{<} \rightarrow \Delta$. Therefore (2.17) induces equivalences

$$
\Delta_{ \pm} \cong \Delta^{<o}, \quad \Delta_{ \pm}^{o} \cong \Delta^{<}
$$

This observation is sometimes called the Joyal duality, although we have not been able to trace the origin of the name (nor of the observation).

Now, the concatenation product - o - obviosly extends to the category $\Delta^{<}$, and it turns out that it is simpler to first describe the corresponding monoidal structure on the opposite category $\Delta^{<o}$. We have $B \Delta^{<o} \cong \operatorname{ar}^{ \pm}(\Delta)$, with the fibration $s: \operatorname{ar}^{ \pm}(\Delta) \rightarrow \Delta$ of Example 1.15, and the identification $B \Delta_{[1]}^{<o}=\Delta_{ \pm} \cong \Delta^{<o}$ is (2.18). In terms of the bispecial category $\Delta_{ \pm}$, the product is given by the reduced concatenation $[n] *[m]=[n] \sqcup_{[0]}[m]$, where the coproduct is taken with respect to the embeddings $t:[0] \rightarrow[n]$, $s:[0] \rightarrow[m]$ (so that what we consider is a cocartesian square (2.1)).

Any monoidal structure $B \mathcal{C}$ on a category $\mathcal{C}$ induces a monoidal structure $B \mathcal{C}^{o}=(B \mathcal{C})^{\tau}=(B \mathcal{C})_{\perp}^{o}$ on the opposite category $\mathcal{C}^{o}$, so that we also obtain the monoidal structure on $\Delta^{<}$. However, it also has a more direct description in terms of the embedding $\lambda_{b}: \Delta^{<} \rightarrow \Delta_{ \pm}$. Namely, note that this embedding is injective on both objects and morphisms, and its image consists of 
objects $[n] \in \Delta_{ \pm}$such that the unique bispecial map [1] $\rightarrow[n]$ is injective (that is, $n \geq 1$ ), and bispecial maps $f:[n] \rightarrow[m]$ such that $f^{-1}(0)=0$ and $f^{-1}(n)=m$. We can then consider the subcategory $\operatorname{ar}^{c}(\Delta) \subset \operatorname{ar}(\Delta)$ spanned by injective bispecial arrows and maps between them represented by commutative squares (1.9) that are also cartesian. One checks that the projection $s: \operatorname{ar}^{c}(\Delta) \rightarrow \Delta$ is a fibration, and we have $B \Delta<\cong \operatorname{ar}^{c}(\Delta)$.

Since concatenation and reduced concatenation are different, the functors $\rho_{b}: \Delta_{ \pm} \rightarrow \Delta \subset \Delta^{<}, \lambda_{b}: \Delta^{<} \rightarrow \Delta_{ \pm}$are not monoidal. However, we do have an obvious functorial map $[m] \circ[n] \rightarrow[m] *[n],[n],[m] \in \Delta$ from the usual concatenation to the reduced one, and this is an example of the following useful 2-categorical structure.

Definition 2.16. For any 2-categories $\mathcal{C}_{0}, \mathcal{C}_{1}$, a co-lax 2 -functor from $\mathcal{C}_{0}$ to $\mathcal{C}_{1}$ is a functor $\gamma: \mathcal{C}_{0} \rightarrow \mathcal{C}_{1}$ over $\Delta$ that is cartesian over all anchor maps, and a lax 2-functor is a co-lax 2-functor $\gamma: \mathcal{C}_{0}^{\tau} \rightarrow \mathcal{C}_{1}^{\tau}$ between the 2-opposite 2 -categories.

For strict 2-categories, one can describe lax and co-lax 2-functors in terms of Definition 1.2. for a lax 2-functor $\gamma$, one allows the maps $\gamma_{c}, \gamma_{c, c^{\prime}, c^{\prime \prime}}$ that are not invertible, and for a co-lax $\gamma$, they also go in the opposite direction. In terms of Definition 2.5, these maps are the maps (1.5) for the transition functors (2.5) and (2.4). A co-lax monoidal structure on a functor $\gamma: \mathcal{C} \rightarrow \mathcal{C}^{\prime}$ between monoidal categories $\mathcal{C}, \mathcal{C}^{\prime}$ is a co-lax 2-functor $B \gamma: B \mathcal{C} \rightarrow B \mathcal{C}^{\prime}$, and a lax monoidal structure on $\gamma$ is a co-lax one on $\gamma^{o}$.

If $\gamma: \mathcal{C}_{0} \rightarrow \mathcal{C}_{1}$ is a co-lax 2-functor such that $\gamma([0])$ is an isomorphism and $\gamma([1])$ admits a right-adjoint $\gamma([1])^{\dagger}$, then for any $[n] \in \Delta, \gamma([n])$ admits a right-adjoint $\gamma([n])^{\dagger}$ by virtue of the Segal condition. In such a situation, Example 1.10 provides a functor $\gamma_{\perp}^{\dagger}$ whose opposite $\gamma^{\dagger}: \mathcal{C}_{1}^{\tau} \rightarrow \mathcal{C}_{0}^{\tau}$ is a lax 2 -functor from $\mathcal{C}_{1}$ to $\mathcal{C}_{0}$. In particular, if we have monoidal categories $\mathcal{C}, \mathcal{C}^{\prime}$ and functor $\gamma: \mathcal{C} \rightarrow \mathcal{C}^{\prime}$ that admits a right-adjoint $\gamma^{\dagger}$, then a co-lax structure $B \gamma$ on $\gamma$ induces a lax structure $B \gamma^{\dagger}=(B \gamma)^{\dagger}$ on $\gamma^{\dagger}$, and vice versa.

With these definitions, the embedding $\lambda_{b}: \Delta^{<} \rightarrow \Delta_{ \pm}$has a natural co-lax monoidal structure given by the embedding $\operatorname{ar}^{c}(\Delta) \subset \operatorname{ar}^{b}(\Delta)$, and the right-adjoint embedding $\rho_{b}: \Delta_{ \pm} \rightarrow \Delta \subset \Delta^{<}$then has a lax monoidal structure by adjunction. However, the two structures are actually the same, since under the identifications (2.18), we have $\rho_{b} \cong \lambda_{b}^{o}$. 


\section{Adjunction.}

3.1 Adjoint pairs. For any 2-category $\mathcal{C}$ and two objects $c, c^{\prime} \in \mathcal{C}_{[0]}$, an adjoint pair of maps between $c$ and $c^{\prime}$ is a quadruple $\left\langle f, f^{\vee}, a, a^{\vee}\right\rangle$ of objects $f \in \mathcal{C}\left(c, c^{\prime}\right), f^{\vee} \in \mathcal{C}\left(c^{\prime}, c\right)$ and maps $a: f^{\vee} \circ f \rightarrow \mathrm{id}_{c}, a^{\vee}: \mathrm{id}_{c^{\prime}} \rightarrow f \circ f^{\vee}$, subject to the relations

$$
\left(\operatorname{id}_{f} \circ a\right) \circ\left(a^{\vee} \circ \mathrm{id}_{f}\right)=\mathrm{id}_{f}, \quad\left(a \circ \mathrm{id}_{f} \vee\right)\left(\operatorname{id}_{f} \circ a^{\vee}\right)=\mathrm{id}_{f} \vee
$$

If $\mathcal{C}=\mathcal{C}$ at, then this is the usual definition of an adjoint pair of functors. For any $\mathcal{C}$, we say that $f \in \mathcal{C}\left(c, c^{\prime}\right)$ is reflexive if it extends to an adjoint pair, and we say that a reflexive morphism $f \in \mathcal{C}\left(c, c^{\prime}\right)$ is an equivalence if there exists an adjoint pair $\left\langle f, f^{\vee}, a, a^{\vee}\right\rangle$ with invertible $a$ and $a^{\vee}$. We note that all the usual properties of adjunction in $\mathcal{C}$ at extend to an arbitrary $\mathcal{C}$ : reflexivity is closed under compositions, the adjoint $f^{\vee}$ to a reflexive $f$ is unique up to a unique isomorphism, and $f$ is an equivalence if and only if there exists $f^{\vee} \in \mathcal{C}\left(c^{\prime}, c\right)$ such that id ${ }_{c} \cong f^{\vee} \circ f$ and $\mathrm{id}_{c^{\prime}} \cong f \circ f^{\vee}$ (to reduce the general case to the case $\mathcal{C}=\mathcal{C} a t$, one can use the Yoneda embedding of Theorem 2.12, and this also works for large $\mathcal{C}$ by Remark 2.14).

Remark 3.1. The above definition is almost the same as in [K3, Subsection 7.1], but with one difference: here $f^{\vee}$ is actually left-adjoint to $f$, while in [K3] it is right-adjoint. The reason for the discrepancy is the change of variance mentioned in Remark 2.6.

By Example 2.1, we have $\operatorname{Fun}^{2}([1], \mathcal{C}) \cong \mathcal{C}_{[1]}$ for any 2-category $\mathcal{C}$, so that morphisms in $\mathcal{C}$ correspond bijectively to 2 -functors nat $=\Delta[1] \rightarrow \mathcal{C}$. Analogously, equivalences correspond to 2-functors eq $=\Delta e(\{0,1\}) \rightarrow \mathcal{C}$. The tautological embedding [1] $\rightarrow e(\{0,1\})$ defines a 2 -functor $\beta$ : nat $\rightarrow$ eq, and a morphism is an equivalence if and only if the corresponding 2 -functor nat $\rightarrow \mathcal{C}$ factors through $\beta$. It turns out that one can reasonably explicitly construct a factorization

$$
\text { nat } \stackrel{\delta}{\longrightarrow} \text { adj } \stackrel{\nu}{\longrightarrow} \text { eq }
$$

of the 2-functor nat $\rightarrow$ eq and a 2-category adj that classifies adjoint pairs in the same way.

To do this, note that by definition, eq $=\Delta e(\{0,1\})$ is the category of pairs $\langle[n], e\rangle,[n] \in \Delta, e:[n] \rightarrow e(\{0,1\})$ a functor (or equivalently, a map of sets $[n] \rightarrow\{0,1\})$. Then we have functorial subsets $[n]_{l}=e^{-1}(l) \subset[n]$, $l=0,1$. Say that a map $f:[n] \rightarrow[m]$ in eq is $l$-special, $l=0,1$, if $f:[n]_{l} \rightarrow[m]_{l}$ is an isomorphism. Then the classes 0 and 1 of 0 -special 
and 1-special maps in eq are closed and form a factorization system in eq in the sense of Example 1.15, in either order (for a formal proof of this, see [K3, Lemma 7.4]). Moreover, if $f$ is bispecial, then so are both of the components of the corresponding decomposition (1.10). Then the anchor/bispecial factorization system on $\Delta$ lifts to eq by Example 1.15, and any map $f:[n] \rightarrow[m]$ in eq uniquely factors as

$$
[n] \stackrel{b_{1}}{\longrightarrow}\left[n^{\prime}\right] \stackrel{b_{0}}{\longrightarrow}\left[n^{\prime \prime}\right] \stackrel{a}{\longrightarrow}[m],
$$

with bispecial 1 -special $b_{1}$, bispecial 0 -special $b_{0}$, and anchor $a$. This means that in particular, the class $\pm \cap 1$ of bispecial 1-special maps also fits into a factorization system, and the projection $s: \operatorname{ar}^{ \pm \cap 1}(\mathrm{eq}) \rightarrow$ eq is a fibration by Example 1.15. We let $\operatorname{adj}=\operatorname{ar}^{ \pm \cap 1}$ (eq). It is fibered over eq, hence over $\Delta$, and it is immediate to check that adj is a 2-category, and $s$ then becomes a 2functor $\nu$ of (3.2). The projection $\nu$ has an obvious fully faithful left-adjoint $\eta:$ eq $\rightarrow$ adj sending $\langle[n], e\rangle$ to the identity arrow id : $\langle[n], e\rangle \rightarrow\langle[n], e\rangle$. While it does not have a right-adjoint, we can nevertheless define a 2 -subcategory $\operatorname{adj}^{0} \subset$ adj by the cartesian square

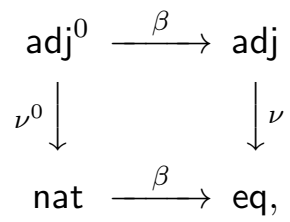

and $\nu^{0}$ has both a left-adjoint $\eta^{0}$ induced by $\eta$, and a right-adjoint $\delta^{0}$. We can then define the other 2-functor in (3.2) by $\delta=\beta \circ \delta^{0}$.

Explicitly, the 2-category adj has two objects 0,1 , and by definition, the endomorphism category $\operatorname{adj}(0,0)$ with its monoidal structure is the category $\Delta_{ \pm}$with the monoidal structure $B \Delta_{ \pm}=\operatorname{ar}^{b}(\Delta)$ of Subsection 2.4. A moment's reflection shows that $\operatorname{adj}(1,1)$ is the opposite category $\Delta^{<}$with the monoidal structure $B \Delta^{<} \cong \operatorname{ar}^{c}(\Delta) \subset \operatorname{ar}^{b}(\Delta)$. Altogether, we have

$$
\operatorname{adj}(1,1)=\Delta^{<}, \operatorname{adj}(1,0)=\Delta_{-}, \operatorname{adj}(0,1)=\Delta_{+}, \operatorname{adj}(0,0)=\Delta_{ \pm},
$$

with compositions

$$
\Delta^{<} \times \Delta_{-} \rightarrow \Delta_{-}, \Delta_{+} \times \Delta^{<} \rightarrow \Delta_{+}, \Delta^{<} \times \Delta^{<} \rightarrow \Delta^{<}, \Delta_{+} \times \Delta_{-} \rightarrow \Delta_{ \pm}
$$

given by the concatenation product $-\circ-$, and compositions

$$
\Delta_{ \pm} \times \Delta_{+} \rightarrow \Delta_{+}, \Delta_{-} \times \Delta_{ \pm} \rightarrow \Delta_{-}, \Delta_{ \pm} \times \Delta_{ \pm} \rightarrow \Delta_{ \pm}, \Delta_{-} \times \Delta_{+} \rightarrow \Delta^{<}
$$


given by the reduced concatenation product $-*-$. Then we have two morphisms $\mathrm{f}: 0 \rightarrow 1, \mathrm{f}^{\vee}: 1 \rightarrow 0$ in adj corresponding to the initial objects in $\Delta_{+}, \Delta_{-}$, and $f^{\vee} \circ f \cong[0] \in \Delta^{<o} \cong \Delta_{ \pm}, f \circ f^{\vee} \cong[0] \in \Delta^{<}$, so that the unique map $o=\emptyset \rightarrow[0]$ produces maps a $: \mathrm{f}^{\vee} \circ \mathrm{f} \rightarrow \mathrm{id}_{0}, \mathrm{a}^{\vee}: \mathrm{id}_{1} \rightarrow \mathrm{f} \circ \mathrm{f}^{\vee}$.

Lemma 3.2. The quadruple $\left\langle\mathrm{f}, \mathrm{f}^{\vee}, \mathrm{a}, \mathrm{a}^{\vee}\right\rangle$ is an adjoint pair in the 2-category adj, and for any adjoint pair $\left\langle f, f^{\vee}, a, a^{\vee}\right\rangle$ in a 2-category $\mathcal{C}$, there exists a 2-functor $\gamma:$ adj $\rightarrow \mathcal{C}$ and an isomorphism $\gamma\left(\left\langle\mathrm{f}, \mathrm{f}^{\vee}, \mathrm{a}, \mathrm{a}^{\vee}\right\rangle\right) \cong\left\langle f, f^{\vee}, a, a^{\vee}\right\rangle$, unique up to a unique isomorphism.

Proof. Both compositions in (3.1) for the quadruple $\left\langle\mathrm{f}, \mathrm{f}^{\vee}, \mathrm{a}^{\mathrm{3}}, \mathrm{a}^{\vee}\right\rangle$ are given by the composition $e \circ s:[0] \rightarrow[1] \rightarrow[0]$ in $\Delta_{+} \cong \Delta_{-}^{o}$, and since $e \circ s=\mathrm{id}$, we indeed have an adjoint pair in adj. Since all the categories in (3.5) have no non-trivial isomorphisms, the 2-category adj is strict, and the underlying 1-category Adj is simply the path category of the wheel quiver with two vertices 0,1 and two edges $f, f^{\vee}$. Therefore by [K3, Lemma 6.15], for any $\mathcal{C}$ and adjoint pair $\left\langle f, f^{\vee}, a, a^{\vee}\right\rangle$, we have a unique 2-functor $\gamma$ from Adj to $\mathcal{C}$ sending $\mathrm{f}$ to $f$ and $\mathrm{f}^{\vee}$ to $f^{\vee}$, and we need to extend it to adj $\supset \Delta$ Adj. In other words, we need to define $\gamma$ on all the morphisms in the categories (3.5). The morphisms in $\Delta^{<}=\operatorname{adj}(1,1)$ are generated by the surjective degeneracy maps $s_{n}^{l}:[n+1] \rightarrow[n]$ and injective face maps $d_{n}^{l}:[n-1] \rightarrow[n], l \in[n]$, and all the maps $\gamma\left(d_{n}^{l}\right), \gamma\left(s_{n}^{l}\right)$ are uniquely defined by $\gamma\left(d_{0}^{0}\right)=\gamma\left(\mathrm{a}^{\vee}\right)=$ $a^{\vee}$ and $\gamma\left(s_{0}^{0}\right)=\gamma\left(\mathrm{id}_{\mathrm{f}} \circ \mathrm{a} \circ \mathrm{id}_{f^{\vee}}\right)=\mathrm{id}_{f} \circ \mathrm{a} \circ \mathrm{id}_{f} \vee$. The fact that (3.1) then yields all the relations between the face and degeneracy maps is well-known (when $\mathcal{C}=\mathcal{C}$ at, it just means that $f \circ \mathrm{f}^{\vee}$ is a comonad, that is, a counital coassociative coalgebra in $\mathcal{C}(1,1))$. This defines $\gamma$ on adj $(1,1)$. For the other three morphism categories (3.5), note that they admit faithful embeddings into $\operatorname{adj}(1,1)$ given by $f \circ-,-\circ f^{\vee}, f \circ-\circ f^{\vee}$, and the morphisms that are in the images of these embeddings are again generated by face and degeneracy maps, modulo the same relations as in the ambient category $\Delta^{<}$. Therefore $a$ and $a^{\vee}$ also uniquely define $\gamma$ on $\operatorname{adj}(1,0), \operatorname{adj}(0,1), \operatorname{adj}(0,0) \subset \operatorname{adj}(1,1)$.

Remark 3.3. Note that the Joyal duality (2.18) identifies the 2-category adj with its 2-opposite 2-category $\operatorname{adj}^{\tau}$ (the equivalence adj $\cong \operatorname{adj}^{\tau}$ interchanges the objects 0 and 1 ). We of course also have eq $\cong \mathrm{eq}^{\tau}$.

Remark 3.4. One can also distinguish an intermediate class between adjoint pairs and equivalences, by requiring that $a^{\vee}$ but not necessairly $a$ is invertible. In $\mathcal{C}$ at, this amounts to saying that the adjoint $f^{\vee}$ to the reflexive functor $f$ is fully faithful. The corresponding universal 2-category is 
then the full subcategory $\operatorname{adj}^{p} \subset$ adj spanned by surjective bispecial 1-special maps. While the embedding $\operatorname{adj}^{p} \subset$ adj is only a co-lax 2-functor, it has an honest right-adjoint 2 -functor $\operatorname{adj} \rightarrow \operatorname{adj}^{p}$ that allows one to fit $\operatorname{adj}^{p}$ into the space in (3.2) between adj and eq. The 2-category $\operatorname{adj}^{p}$ appeared in [K3, Subsection 8.5] but only in passing; it might be interesting to explore its combinatorics.

3.2 Co-adjoint pairs and twisting. Once we have constructed the universal 2-categories (3.2), we can define morphisms, adjoint pairs and equivalences between 2 -functors in the same way as in (1.2). Namely, assume given 2-categories $\mathcal{C}, \mathcal{C}^{\prime}$ and 2-functors $\gamma_{0}, \gamma_{1}: \mathcal{C} \rightarrow \mathcal{C}^{\prime}$. Then a 1-morphism, or a natural transformation from $\gamma_{0}$ to $\gamma_{1}$ is a 2 -functor

$$
\gamma: \mathcal{C} \times{ }^{2} \text { nat } \rightarrow \mathcal{C}^{\prime}
$$

that restricts to $\gamma_{l}$ on $\mathcal{C} \times\{l\} \subset \mathcal{C} \times{ }^{2}$ nat, $l=0,1$, and similarly, an adjoint pair and a pair of adjoint equivalences are 2-functors

$$
\gamma: \mathcal{C} \times^{2} \text { adj } \rightarrow \mathcal{C}^{\prime}, \quad \gamma: \mathcal{C} \times{ }^{2} \text { eq } \rightarrow \mathcal{C}^{\prime} .
$$

However, our explicit construction of the 2-category adj also reveals some additional structure that is not obvious from the definitions. Namely, we also have a projection $t: \operatorname{adj} \cong \operatorname{ar}^{ \pm \cap 1}($ eq $) \rightarrow$ eq $\rightarrow \Delta$ sending an arrow to its target, and for any 2 -category $\mathcal{C}$, we can define a category $\mathcal{C}\{\operatorname{adj}\}$ by the cartesian square

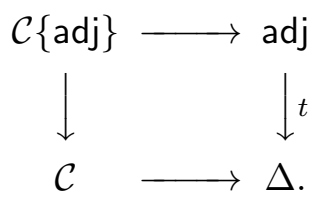

The structural fibration $s:$ adj $\rightarrow \Delta$ then provides a fibration $\mathcal{C}\{\operatorname{adj}\} \rightarrow \Delta$, and it is elementary to check that this turns $\mathcal{C}\{\operatorname{adj}\}$ into a 2-category.

Definition 3.5. for any 2-categories $\mathcal{C}, \mathcal{C}^{\prime}$, a coadjoint pair of 2-functors from $\mathcal{C}$ to $\mathcal{C}^{\prime}$ is a 2 -functor

$$
\gamma: \mathcal{C}\{\operatorname{adj}\} \rightarrow \mathcal{C}^{\prime}
$$

If $\mathcal{C} \cong \mathcal{C}_{[0]} \times \Delta$ is discrete, then $\mathcal{C}\{\operatorname{adj}\} \cong \mathcal{C} \times{ }^{2}$ adj, so that adjoint and coadjoint pairs defined on $\mathcal{C}$ coincide. It turns out that in general, co-adjoint pairs are much easier to control. Namely, note that any 2-functor $\mathcal{C} \rightarrow \mathcal{C}^{\prime}$ 
with discrete $\mathcal{C}^{\prime}$ trivially satisfies the assumptions on Lemma 1.11, so that it is a fibration. For any 2-category $\mathcal{C}$ equipped with a 2 -functor $\mathcal{C} \rightarrow$ eq, say that a map in $\mathcal{C}$ is $l$-special, $l=0,1$, if it is a cartesian lifting of an $l$-special map in eq. For any 2-category $\mathcal{C}$, denote $\mathcal{C}\{$ eq $\}=\mathcal{C} \times{ }^{2}$ eq, with its projection $\mathcal{C}\{$ eq $\} \rightarrow$ eq, and say that a co-lax 2 -functor $\mathcal{C} \rightarrow \mathcal{C}^{\prime}$ is $l$-special if it sends $l$-special maps in $\mathcal{C}\{$ eq $\}$ to cartesian maps in $\mathcal{C}^{\prime}$. Now, as we have mentioned, the functor $\nu$ of (3.2) has a left-adjoint $\eta:$ eq $\rightarrow$ adj, and for any $\mathcal{C}$, these induce an adjoint pair of functors

$$
\nu: \mathcal{C}\{\operatorname{adj}\} \rightarrow \mathcal{C}\{\text { eq }\}, \quad \eta: \mathcal{C}\{\text { eq }\} \rightarrow \mathcal{C}\{\operatorname{adj}\}
$$

where $\nu$ is a 2-functor, and $\eta$ is a co-lax 2-functor by adjunction. We then have the following result.

Lemma 3.6 ([K3, Lemma 7.4]). For any 2-category $\mathcal{C}$, the co-lax 2-functor $\eta$ of (3.9) is 0 -special, and any 0 -special functor $\gamma: \mathcal{C}\{$ eq $\} \rightarrow \mathcal{C}^{\prime}$ uniquely factors as $\gamma \cong \gamma^{\prime} \circ \eta$ for a unique co-adjoint pair $\gamma^{\prime}: \mathcal{C}\{\operatorname{adj}\} \rightarrow \mathcal{C}^{\prime}$.

Lemma 3.6 essentially says that a co-adjoint pair is completely defined by the corresponding 0 -special co-lax 2-functor, and there is also one general result proved in [K3, Subsection 7.4] that helps to construct such 2-functors. To state it, we need some preliminaries. For any co-lax 2-functor $\gamma: \mathcal{C}^{\prime} \rightarrow \mathcal{C}$, one can define a 2 -category $\gamma^{*} \mathcal{C}$ by the cartesian square

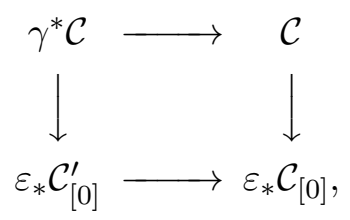

where as in (2.7), $\varepsilon: \mathrm{pt} \rightarrow \Delta$ is the embedding onto $[0] \in \Delta$. Informally, $\gamma^{*}$ has the same objects as $\mathcal{C}^{\prime}$ and the same morphisms as $\mathcal{C}$. Then $\gamma$ factors as

$$
\mathcal{C}^{\prime} \stackrel{\widetilde{\gamma}}{\longrightarrow} \gamma^{*} \mathcal{C} \stackrel{\bar{\gamma}}{\longrightarrow} \mathcal{C},
$$

and in keeping with our usage for the usual categories, we say that $\gamma$ is dense resp. full if $\bar{\gamma}$ resp. $\widetilde{\gamma}$ is an equivalence. A 2-subcategory $\mathcal{C}^{\prime} \subset \mathcal{C}$ is full resp. dense iff so is the embedding 2-functor $\mathcal{C}^{\prime} \rightarrow \mathcal{C}$. For example, if $\gamma: \mathcal{C}_{[0]}^{\prime} \times \Delta \rightarrow \mathcal{C}_{[0]} \times \Delta \rightarrow \mathcal{C}$ is the embedding 2-functor for some $\mathcal{C}_{[0]}^{\prime} \subset \mathcal{C}_{[0]}$, then $\gamma^{*} \mathcal{C} \subset \mathcal{C}$ is the full 2-subcategory spanned by objects $c \in \mathcal{C}_{[0]}^{\prime}$.

Proposition 3.7 ([K3, Proposition 7.9]). Assume given some 2-categories $\mathcal{C}^{\prime}, \mathcal{C}$, denote by $\iota_{1}: \mathcal{C}^{\prime} \rightarrow \mathcal{C}^{\prime}\{$ eq $\}$ the embedding onto $\mathcal{C}^{\prime} \times\{1\} \subset \mathcal{C}^{\prime}\{$ eq $\}$, 
and assume given a 0-special co-lax 2-functor $\gamma: \mathcal{C}^{\prime}\{\mathrm{eq}\} \rightarrow \mathcal{C}$. Then the component $\widetilde{\gamma}$ of its decomposition (3.10) factors as

$$
\mathcal{C}^{\prime}\{\text { eq }\} \stackrel{\widetilde{\gamma}_{1} \times \text { id }}{\longrightarrow} \gamma_{1}^{*} \mathcal{C}\{\text { eq }\} \stackrel{\gamma^{\diamond}}{\longrightarrow} \gamma^{*} \mathcal{C},
$$

where $\gamma_{1}=\gamma \circ \iota_{1}, \widetilde{\gamma}_{1}: \mathcal{C}^{\prime} \rightarrow \gamma_{1}^{*} \mathcal{C}$ is as in (3.10), and $\gamma^{\diamond}$ is a 0 -special co-lax 2 -functor over eq $/ \Delta^{o}$ equipped with an isomorphism $\iota_{1}^{*}\left(\gamma^{\diamond}\right) \cong \mathrm{id}$. Moreover, such a factorization is unique up to a unique isomorphism.

In particular, Proposition 3.7 provides the "twisting co-lax 2-functor" $\Theta$ from $\gamma_{1}^{*} \mathcal{C}$ to $\mathcal{C}$ obtained by restricting $\gamma^{\diamond}$ to $\gamma_{1}^{*} \mathcal{C} \times\{0\} \subset \gamma_{1}^{*} \mathcal{C}\{$ eq $\}$. Explicitly, for any $c \in \mathcal{C}_{[0]}^{\prime}, \gamma$ gives rise to an adjoint pair $\gamma_{c}:$ adj $\rightarrow \mathcal{C}$ consisting of objects $\gamma_{0}(c), \gamma_{1}(c) \in \mathcal{C}_{[0]}$, morphisms $h_{c} \in \mathcal{C}\left(\gamma_{0}(c), \gamma_{1}(c)\right), h_{c}^{\vee} \in \mathcal{C}\left(\gamma_{1}(c), \gamma_{0}(c)\right)$, and adjunction maps between their compositions. Then $\Theta$ sends $c$ to $\gamma_{0}(c)$, and on morphisms, it is given by

$$
\Theta(g)=h_{c^{\prime}}^{\vee} \circ g \circ h_{c}, \quad g \in\left(\gamma_{1}^{*} \mathcal{C}\right)\left(c, c^{\prime}\right) \cong \mathcal{C}\left(\gamma_{1}(c), \gamma_{1}\left(c^{\prime}\right)\right) .
$$

The maps (1.5) are induced by the adjunction maps between $h$. and $h_{\bullet}^{\vee}$.

Example 3.8. For a somewhat trivial but useful application of Proposition [3.7. assume given a 2-category $\mathcal{C}$ with a full 2-subcategory $\mathcal{C}^{\prime} \subset \mathcal{C}$, and assume that for any $c \in \mathcal{C}_{[0]}^{\prime} \subset \mathcal{C}_{[0]}$, we are given an equivalent object $\theta(c) \in \mathcal{C}_{[0]}$ - that is, a 2-functor $\gamma_{c}:$ eq $\rightarrow \mathcal{C}$ sending 1 to $c$ and 0 to some $\theta(c)$. Then the functors $\gamma_{c}$ together define a 2-functor $\gamma: \mathcal{C}_{[0]}^{\prime} \times$ eq $\rightarrow \mathcal{C}$ such that $\gamma_{1}^{*} \mathcal{C} \cong \mathcal{C}^{\prime}$, and then Proposition 3.7 provides a co-lax 2-functor $\Theta: \mathcal{C}^{\prime} \rightarrow \mathcal{C}, c \mapsto \theta(c)$. By (3.12), since we are dealing with equivalences and not just adjoint pairs, $\Theta$ is in fact a 2 -functor and a full embedding $\mathcal{C}^{\prime} \rightarrow \mathcal{C}$ different from the original one. However, the two embeddings are equivalent in the sense of (3.7), with the equivalence provided by $\gamma^{\diamond}$ (that in this case is also a 2-functor). Note that the equivalences $\gamma_{c}$ are chosen separately and independently for each $c$, and do not need to be compatible with morphisms in $\mathcal{C}^{\prime}$ in any way.

3.3 Iterated adjoint pairs. Let us now describe a non-trivial application of Proposition [3.7 given in [K3, Subsection 7.5]. It concerns compositions of reflexive morphisms and adjoint pairs.

For any objects $c, c^{\prime} \in \mathcal{C}_{[0]}$ in a 2-category $\mathcal{C}$, adjoint pairs of maps between $c$ to $c^{\prime}$ form a category $\mathcal{A} d j(\mathcal{C})\left(c, c^{\prime}\right)$. In fact, the category is a groupoid, and the forgetful functor $\mathcal{A d j}\left(c, c^{\prime}\right) \rightarrow \mathcal{C}\left(c, c^{\prime}\right)_{\text {Iso }},\left\langle f, f^{\vee}, a, a^{\vee}\right\rangle \mapsto f$ 
is fully faithful, so we may identify $\mathcal{A} d j(\mathcal{C})\left(c, c^{\prime}\right)$ with its essential image and treat is a subcategory in $\mathcal{C}\left(c, c^{\prime}\right)$. This is a stronger form of the usual uniqueness of adjoints; for $\mathcal{C}=\mathcal{C} a t$, it is easy to check it directly, and the general case again reduces to $\mathcal{C}$ at by the Yoneda embedding of Theorem 2.12, Equivalences then span a full subcategory $\mathcal{E} q(\mathcal{C})\left(c, c^{\prime}\right) \subset \mathcal{A} d j(\mathcal{C})\left(c, c^{\prime}\right)$. Since both reflexive morphisms and equivalences are closed under compositions, we in fact have dense 2 -subcategories

$$
\mathcal{E} q(\mathcal{C}) \subset \mathcal{A} d j(\mathcal{C}) \subset \mathcal{C}
$$

with morphism categories $\mathcal{E} q(\mathcal{C})(-,-), \mathcal{A} d j(\mathcal{C})(-,-)$, and moreover, by Lemma 3.2, we have $\mathcal{A} d j(\mathcal{C})_{[1]} \cong \operatorname{Fun}^{2}(\operatorname{adj}, \mathcal{C})$ and $\mathcal{E} q(\mathcal{C})_{[1]} \cong \operatorname{Fun}^{2}($ eq, $\mathcal{C})$. Our goal is a similar universal description for the whole 2-categories (3.13).

To package the answer, it is convenient to say that a 2 -kernel is a small category $\mathcal{K}$ equipped with a fibration $\mathcal{K} \rightarrow \Delta^{o} \times \Delta$ such that for any $[n] \in \Delta^{o}$, $\mathcal{K}_{[n]} \rightarrow \Delta$ is a 2 -category (this is a version of " $\Delta$-kernels" used in [K3, Subsection 7.5]). For any 2-kernel $\mathcal{K}$ and 2-category $\mathcal{C}$, we then have a fibration $\operatorname{Fun}^{2}(\mathcal{K}, \mathcal{C}) \rightarrow \Delta$ with fibers $\operatorname{Fun}^{2}(\mathcal{K}, \mathcal{C})_{[n]} \cong \operatorname{Fun}^{2}\left(\mathcal{K}_{[n]}, \mathcal{C}\right)$ and transition functors given by pullbacks with respect to the transition functors $\mathcal{K}_{[n]} \rightarrow \mathcal{K}_{[m]}$ of the fibration $\mathcal{K} \rightarrow \Delta^{o}$. We then want to promote (3.2) to a sequence

$$
\text { Nat } \stackrel{\delta}{\longrightarrow} \operatorname{Adj} \stackrel{\nu}{\longrightarrow} \mathrm{Eq}
$$

of 2-kernel and functors cartesian over $\Delta^{o} \times \Delta$ that produces (3.13) after applying $\operatorname{Fun}^{2}(-, \mathcal{C})$.

For Nat, the answer is tautological: we have $\operatorname{Nat}_{[n]} \cong \Delta[n]$ for any $[n]$, and Nat itself is the twisted arrow category $\operatorname{tw}\left(\Delta^{o}\right)$ of Example 1.14, For Eq, it is easy to see that we have $\mathrm{Eq}_{[n]}=\Delta e(\{0, \ldots, n\})$, and if we let $V: \Delta \rightarrow \Gamma$ be the functor sending an ordinal $[n]$ to the finite set $V([n])=\{0, \ldots, n\}$, then $\mathrm{Eq} \cong\left(V^{o} \times V\right)^{*} \operatorname{tw}\left(\Gamma^{o}\right)$, and $V$ induces a cartesian functor $\beta:$ Nat $\rightarrow \mathrm{Eq}$.

To construct Adj, consider the cofibration $t: \operatorname{ar}^{t}(\Delta) \cong \Delta^{*} \rightarrow \Delta$ of Remark 2.10, with the functor $s: \Delta^{\bullet} \rightarrow \Delta,\langle[n], l\rangle \mapsto[n-l]$, and note that if we restrict $s$ to $\theta^{*} \Delta^{*} \subset \Delta^{*}$, where the embedding $\theta: \Delta^{o} \subset \Delta^{<o} \cong \Delta_{ \pm} \subset \Delta$ is induced by the Joyal duality (2.18), then it factors through $\Delta_{-} \subset \Delta$. We can then apply the duality (2.17) and obtain a functor $w^{\prime}: \theta^{*} \Delta^{\bullet} \rightarrow \Delta_{+}^{o} \subset \Delta^{o}$. Moreover, we can modify it by setting

$$
w(\langle[n], l\rangle)= \begin{cases}{[n-l]=w^{\prime}(\langle[n], l\rangle),} & l>0, \\ t([n-1]) \subset[n]=w^{\prime}(\langle[n], l), & l=0,\end{cases}
$$


and we note that this gives a well-defined functor $w: \theta^{*} \Delta^{\bullet} \rightarrow \Delta^{o}$. Indeed, for any map $f:\langle[n], l\rangle \rightarrow\left\langle n^{\prime}, l^{\prime}\right\rangle$ in $\theta^{*} \Delta^{*}$, the map $f$ must be bispecial, so that if $l=0$ then $l^{\prime}=0$, and moreover, $w^{\prime}(f)=f^{\dagger}:\left[n^{\prime}\right] \rightarrow[n]$ sends $t([n-1]) \subset[n]$ into $t\left(\left[n^{\prime}-1\right] \subset\left[n^{\prime}\right]\right.$. Therefore for any $f, w^{\prime}(f):\left[n^{\prime}-l^{\prime}\right] \rightarrow[n-l]$ sends $w\left(\left\langle\left[n^{\prime}\right], l^{\prime}\right\rangle\right) \subset w^{\prime}\left(\left\langle\left[n^{\prime}\right], l^{\prime}\right\rangle\right)$ into $w(\langle[n], l\rangle) \subset w^{\prime}(\langle[n], l\rangle)$, and the functor $w$ given by (3.15) is indeed well-defined. Explicitly, for any $[n] \in \Delta^{o}$, it restricts to a functor $w_{n}:[n+1]^{o}=\left(\theta^{*} \Delta^{*}\right)_{[n]} \rightarrow \Delta^{o}$, and then the opposite functor $w_{n}^{o}:[n+1] \rightarrow \Delta$ is given by the diagram

$$
[n] \stackrel{g_{0}}{\longrightarrow}[n] \stackrel{g_{1}}{\longrightarrow}[n-1] \stackrel{g_{2}}{\longrightarrow} \ldots \stackrel{g_{n-1}}{\longrightarrow}[1] \stackrel{g_{n}}{\longrightarrow}[0]
$$

in $\Delta$, where $g_{0}=\mathrm{id}$, and $g_{l}:[n+1-l] \rightarrow[n-l]$ for $l \geq 1$ sends 0 to 0 and $i \in[n+1-l], i \geq 1$ to $i-1 \in[n-l]$.

Now consider the fibration Eq $\rightarrow \Delta^{o}$, and let $\operatorname{Adj}^{\prime \prime}=\left(\theta^{*} t\right)_{* *} w^{*} \mathrm{Eq}$, where $\theta^{*} t: \theta^{*} \Delta^{*} \rightarrow \Delta^{o}$ is the restriction of the cofibration $t: \Delta^{*} \rightarrow \Delta$, and $\left(\theta^{*} t\right)_{* *}$ is as in (1.12). By definition, Adj" $^{\prime \prime}$ comes equipped with a fibration $\pi: \operatorname{Adj}^{\prime \prime} \rightarrow \Delta^{o}$, and the fiber $\operatorname{Adj}_{[n]}^{\prime \prime}$ over some $[n] \in \Delta^{o}$ is the category of pairs $\left\langle m_{.}, \varphi\right\rangle$ of a functor $m_{\bullet}: \theta([n])=[n+1] \rightarrow \Delta$ and a map $\varphi: V \circ m_{\bullet} \rightarrow V \circ w_{n}^{o}$. Explicitly, $m$. is a diagram

$$
\left[m_{0}\right] \stackrel{f_{0}}{\longrightarrow}\left[m_{1}\right] \stackrel{f_{1}}{\longrightarrow} \ldots \stackrel{f_{n}}{\longrightarrow}\left[m_{n+1}\right],
$$

and for any $l \in[n+1], \varphi$ provides a partition $\left[m_{l}\right]=\left[m_{l}\right]_{0} \sqcup \ldots \sqcup\left[m_{l}\right]_{j}$ into $j$ subsets, where $j=\min (n+1-l, n)$. If $l>0$, then the map $f_{l}$ in (3.17) sends $\left[m_{l}\right]_{i+1}$ into $\left[m_{l+1}\right]_{i}, 0 \leq i<j$, and it sends $\left[m_{l}\right]_{0}$ into $\left[m_{l+1}\right]_{0}$. The map $f_{0}$ simply sends $\left[m_{0}\right]_{i}$ into $\left[m_{1}\right]_{i}$.

We now observe that since $\theta: \Delta^{o} \rightarrow \Delta$ sends all maps to bispecial maps, both $\left[m_{0}\right]$ and $m_{[n+1]}$ in (3.17) are functorial with respect to $\left\langle m_{\bullet}, \varphi\right\rangle$, so that we have two projections $\sigma, \tau: \operatorname{Adj}^{\prime \prime} \rightarrow \Delta$. We let $\operatorname{Adj}^{\prime}=\tau^{-1}([0]) \subset \operatorname{Adj}^{\prime \prime}$ be the full subcategory spanned by pairs $\left\langle m_{\bullet}, \varphi\right\rangle$ with $\left[m_{n+1}\right]=[0]$, and we still have the projection $\sigma: \operatorname{Adj}^{\prime} \rightarrow \Delta$ and the fibration $\pi: \operatorname{Adj}^{\prime} \rightarrow \Delta^{o}$. Moreover, say that a pair $\left\langle m_{.}, \varphi\right\rangle$ is admissible if $f_{l}:\left[m_{l}\right]_{i} \rightarrow\left[m_{l+1}\right]_{g_{l}(i)}$ is an isomorphism whenever $g_{l}(i) \geq 1$ (note that by (3.16), we have $g_{l}(i)=i-1$ if $l \geq 1$ and $g_{l}(i)=i$ if $\left.l=0\right)$. Let $\operatorname{Adj} \subset \operatorname{Adj}^{\prime}$ be the full subcategory of admissible pairs.

Lemma 3.9. The product $\pi \times \sigma:$ Adj $^{\prime} \rightarrow \Delta^{o} \times \Delta$ is a 2-kernel, and so is the induced projection $\operatorname{Adj} \subset \operatorname{Adj}^{\prime} \rightarrow \Delta^{o} \times \Delta$. Moreover, the embedding $\operatorname{Adj} \subset \operatorname{Adj}^{\prime}$ admits a right-adjoint functor $\operatorname{Adj}^{\prime} \rightarrow$ Adj cartesian over $\Delta^{o} \times \Delta$.

Proof. This is essentially [K3, Lemma 7.10] (modulo the change of variance explained in Remark 2.6). 
One further observes that $\left[m_{0}\right]$ in a diagram (3.17) comes equipped with a partition into $n$ subsets, thus defines an object in $\mathrm{Eq}_{[n]}$, and the fibration Adj $\rightarrow \Delta^{o} \times \Delta$ then factors through a functor $\nu:$ Adj $\rightarrow$ Eq cartesian over $\Delta^{o} \times \Delta$. We can then define the subcategory $\operatorname{Adj}^{0} \subset \operatorname{Adj}$ by the cartesian square

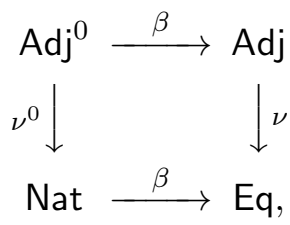

the universal version of (3.4), and $\nu^{0}$ again has a right-adjoint $\delta^{0}$, so we can let $\delta=\beta \circ \delta^{0}$ and obtain our diagram (3.14).

Example 3.10. If $n=0$, then an admissible pair $\left\langle m_{\bullet}, \varphi\right\rangle$ consists of a diagram $[m] \rightarrow[0]$; since $w_{0}$ is the diagram $[0] \rightarrow[0], \varphi$ is unique. Therefore we have $\operatorname{Adj}_{[0]}=\Delta$.

If $n=1$, then we have a diagram $\left[m_{0}\right] \rightarrow\left[m_{1}\right] \rightarrow[0]$, and $\varphi$ defines a partition $\left[m_{1}\right]=\left[m_{1}\right]_{0} \sqcup\left[m_{1}\right]_{1}$ and a partition $\left[m_{0}\right]=\left[m_{0}\right]_{0} \sqcup\left[m_{0}\right]_{1}$. The latter is uniquely defined by the former, and what we have is actually a map $f_{0}$ in eq. Admissibility means that the map 1-special, so that $\operatorname{Adj}_{[1]} \cong$ adj.

We have two maps $s, t:[0] \rightarrow[1]$ in $\Delta$, and the bispecial maps $p=\theta\left(s^{o}\right)$ resp. $q=\theta\left(t^{\circ}\right)$ from [2] to [1] send $1 \in[2]$ to 0 resp. 1. The transition functor $s^{o *}: \Delta \rightarrow$ adj of the fibration $\operatorname{Adj} \rightarrow \Delta^{o}$ then sends a diagram $[m] \rightarrow[0]$ to its pullback $p^{*}$ with respect to $p$ - that is, to the diagram $[m] \rightarrow[m] \rightarrow[0]$, with the first map being the identity map — while $t^{o *}$ sends it to $[m] \rightarrow[0] \rightarrow[0]$, the pullback $q^{*}$ with respect to $q$. The partition data are prescribed by the adjoint maps $p^{\dagger}, q^{\dagger}:[1] \rightarrow[2]$, and we have $p^{\dagger}(1)=2$ and $q^{\dagger}(1)=1$. Therefore $w^{\prime}(p):[1] \rightarrow[2]$ sends 1 to 2 , so that $w(p):[0] \rightarrow[1]$ sends 0 to 1 , while $w(q)$ sends 0 to 0 . This means that for the diagram $[m] \rightarrow[m] \rightarrow[0]$, we take $[m]=[m]_{1}$, while for the diagram $[m] \rightarrow[0] \rightarrow[0]$, we take $[m]=[m]_{0}$. Both diagrams are therefore admissible, and $s^{o *}, t^{o *}$ are the embeddings $\Delta \rightarrow$ adj onto 1 and 0 .

Proposition 3.11 ([[K3, Proposition 7.12]). For any 2-category $\mathcal{C}$, the fibration $\operatorname{Fun}^{2}(\operatorname{Adj}, \mathcal{C}) \rightarrow \Delta$ is a 2-category, and the functor

$$
\delta^{*}: \operatorname{Fun}^{2}(\operatorname{Adj}, \mathcal{C}) \rightarrow \operatorname{Fun}^{2}(\operatorname{Nat}, \mathcal{C}) \cong \mathcal{C}
$$

induced by (3.14) factors through an equivalence $\operatorname{Fun}^{2}(\operatorname{Adj}, \mathcal{C}) \cong \mathcal{A d j}(\mathcal{C})$. 
Let us explain the main idea behind the proof of Proposition 3.11. It uses an alternative inductive construction of the 2-categories $\operatorname{Adj}_{n}=\operatorname{Adj}_{[n]}$ that also explains how can one possibly come up with such a weird object.

Take some $n \geq 2$, consider the cocartesian square (2.1) with $l=1$, and let $e:[n] \rightarrow[1] \times[n-1]$ be the map given by the embedding onto $[1] \times\{0\}$ on $s([1]) \subset[n]$, and by the embedding onto $\{1\} \times[n-1]$ on $t([n-1]) \subset[n]$. Note that $e$ is a map of partially ordered sets, thus a functor, and it has a left-adjoint functor $p:[1] \times[n-1] \rightarrow[n]$ sending $i \times j$ to $i$ if $j=0$ and to $j+1$ if $j \geq 1$. Consider the 2-category $\operatorname{Adj}_{n-1}$ and the corresponding 2-category $\operatorname{Adj}_{n-1}\{\operatorname{adj}\}$ of (3.8). Then by definition, an object in $\operatorname{Adj}_{n-1}\{\operatorname{adj}\}$ is a diagram (3.17) equpped with two types of partition data. Firstly, $f_{0}:\left[m_{0}\right] \rightarrow\left[m_{1}\right]$ defines an object in adj, so that we have a map $\varphi_{1}: V\left(\left[m_{1}\right]\right) \rightarrow\{0,1\}$ (and the induced map $\left.\varphi_{1} \circ V\left(f_{0}\right): V\left(\left[m_{0}\right]\right) \rightarrow\{0,1\}\right)$. Secondly, the part of the diagram that starts from $\left[m_{1}\right]$ defines an object in $\operatorname{Adj}_{n-1}$, so that we have a map $\varphi_{n-1}: V\left(\left[m_{1}\right]\right) \rightarrow\{0, \ldots, n-1\}$. Altogether, we have a map $\varphi_{1} \times \varphi_{n-1}: V\left(\left[m_{1}\right]\right) \rightarrow V([1] \times[n-1])=V([1]) \times V([n-1])$ and the induced map $\left(\varphi_{1} \times \varphi_{n-1}\right) \circ V\left(f_{0}\right): V\left(\left[m_{0}\right]\right) \rightarrow V([1] \times[n-1])$.

Observe that if the image of the map $\varphi_{1} \times \varphi_{n-1}$ lies inside the image of the map $V(e): V([n]) \rightarrow V([1] \times[n-1])$, then we can extend $\varphi_{n-1}$ to a map $\varphi_{n}: V\left(m_{\bullet}\right) \rightarrow V\left(w_{n}^{o}\right)$ by setting $\varphi_{n}=\left(\varphi_{1} \times \varphi_{n-1}\right) \circ V\left(f_{0}\right)$ on $V\left(\left[m_{0}\right]\right), \varphi_{n}=\varphi_{1} \times \varphi_{n-1}$ on $V\left(\left[m_{1}\right]\right)$, and $\varphi_{n}=\varphi_{n-1}$ on $V\left(\left[m_{l}\right]\right)$ for $l \geq 2$. One checks immediately that the admissibility conditions match, so that we obtain a cartesian square

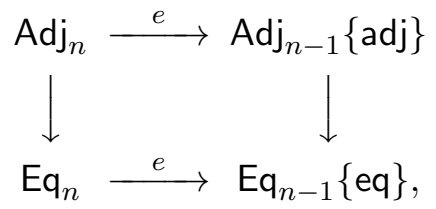

where we identify $\mathrm{Eq}_{n-1}\{$ eq $\} \cong \Delta e(V([1] \times[n]))$. Moreover, the map $p$ extends to a 2-functor $p: \operatorname{Adj}_{n-1}\{\operatorname{adj}\} \rightarrow \operatorname{Adj}_{n}$ such that $p \circ e \cong I d$. Indeed, by Lemma [3.6] and Proposition [3.7, such an extension is uniquely defined by its compositions $p_{i}=p \circ a_{i}$ with the embeddings $a_{i}: \operatorname{adj} \subset \operatorname{Adj}_{n-1}\{\operatorname{adj}\}$ onto $i \times \operatorname{adj}, 0 \leq i \leq n-1$, and we can take $p_{0}$ to be the transition functor $s^{o *}: \operatorname{adj}=\operatorname{Adj}_{1} \rightarrow \operatorname{Adj}_{n}$ of the fibration $\operatorname{Adj} \rightarrow \Delta^{o}$ corresponding to the embedding $s:[1] \rightarrow[n]$, and let $p_{i}, i \geq 1$ correspond to the projection $[1] \rightarrow[0] \rightarrow[n]$ onto $i+1 \in[n]$. We conclude that $\operatorname{Adj}_{n}$ is a retract of the 2-category $\operatorname{Adj}_{n-1}\{\operatorname{adj}\}$.

Now, by Example 3.10 , we know that $\mathcal{A d j}(\mathcal{C}) \cong \operatorname{Fun}^{2}(\operatorname{Adj}, \mathcal{C})$ over the objects [0] and [1] in $\Delta$, so to prove Proposition [3.11, it suffices to check 
that $\operatorname{Fun}^{2}(\operatorname{Adj}, \mathcal{C})$ satisfies the Segal condition. Moreover, by induction, it suffices to check it for a square (2.1) with $l=1$, assuming that we know that $\mathcal{A d j}(\mathcal{C})_{[i]} \cong \operatorname{Fun}^{2}\left(\operatorname{Adj}_{i}, \mathcal{C}\right)$ for $i \leq n-1$. What we have to do, then, is to take a 2-functor $\gamma_{1}:$ adj $\rightarrow \mathcal{C}$ and a 2-functor $\gamma_{n-1}: \operatorname{Adj}_{n-1} \rightarrow \mathcal{C}$ such that $\gamma_{1}(1)=\gamma_{n-1}(0)$ is the same object $c \in \mathcal{C}_{[0]}$, and show that there exists a unique 2-functor $\gamma_{n}: \operatorname{Adj}_{n} \rightarrow \mathcal{C}$ equipped with isomorphisms $\gamma_{n} \circ s^{o *} \cong \gamma_{1}$ and $\gamma_{n} \circ t^{o *} \cong \gamma_{n-1}$. However, again by Lemma 3.6 and Proposition 3.7. for any $(n-1)$-tuple of adjoint pairs $\gamma_{i}^{\prime}: \operatorname{adj} \rightarrow \mathcal{C}$ sending $1 \in \operatorname{adj}_{[1]}$ to the object $\gamma_{n-1}(i) \in \mathcal{C}_{[0]}$, the 2-functor $\gamma_{n-1}$ extends uniquely to a co-adjoint pair $\gamma: \operatorname{Adj}_{n-1}\{\operatorname{adj}\} \rightarrow \mathcal{C}$ equipped with isomorphisms $\gamma \circ a_{i} \cong \gamma_{i}^{\prime}$. It remains to observe that $\gamma$ factors through the retract $\operatorname{Adj}_{n}$ of the 2-category $\operatorname{Adj}_{n-1}\{\operatorname{adj}\}$ if and only if for any $i \geq 1, \gamma_{i}^{\prime}$ factors through the embedding $\Delta \rightarrow \mathcal{C}$ onto $\gamma_{n-1}(i)$, and take $\gamma_{0}^{\prime}=\gamma_{1}$.

\section{Applications.}

4.1 Symmetric monoidal structures. In order to show how the formalism we have developed applies to real life, it is convenient to start with yet another piece of formalism - namely, with a description of symmetric monoidal categories. Traditionally, these are defined in terms of associativity and commutativity isomorphisms that satisfy higher constraints (the pentagon and the hexagon axiom, and the unitality axioms that are often ignored). This theory has no strict version, since the commutativity isomorphism is almost never an identity.

For a description of symmetric monoidal categories in the spirit of Definition 2.5 , let $\Gamma$ be the category of finite sets, and let $\Gamma_{+}$be the category of finite sets and partially defined maps - that is, maps from $S_{0}$ to $S_{1}$ are isomorphism classes of diagrams

$$
S \stackrel{i}{\longleftarrow} \widetilde{S} \stackrel{f}{\longrightarrow} S^{\prime}
$$

in $\Gamma$ with injective $i$, with compositions given by fibered products. Equivalently, $\Gamma_{+}$is the category of finite pointed sets, with the equivalence sending a set $S_{+}$with the distinguished element $o \in S_{+}$to the complement $S=S_{+} \backslash\{o\} \subset S_{+}$, and a map $f: S_{+} \rightarrow S_{+}^{\prime}$ to the diagram (4.1) with $\widetilde{S}=f^{-1}\left(S^{\prime}\right) \subset S$. A map (4.1) in $\Gamma_{+}$is anchor resp. structural if $f$ resp. $i$ is invertible. Coproducts in $\Gamma$ are also coproducts in $\Gamma_{+}$, and for any $S_{0}, S_{1} \in \Gamma_{+}$, we have anchor maps

$$
a_{0}: S_{0} \sqcup S_{1} \rightarrow S_{0}, \quad a_{1}: S_{1} \sqcup S_{1} \rightarrow S_{1}
$$


defined by the embeddings $i_{0}: S_{0} \rightarrow S_{0} \sqcup S_{1}, i_{1}: S_{1} \rightarrow S_{0} \sqcup S_{1}$.

Definition 4.1. A fibration $\mathcal{C} \rightarrow \Gamma_{+}^{o}$ satisfies the Segal condition if $\mathcal{C}_{\emptyset}$ is equivalent to the point category pt, and for any $S_{0}, S_{1} \in \Gamma$, the projection

$$
a_{0}^{*} \times a_{1}^{*}: \mathcal{C}_{S_{0} \sqcup S_{1}} \rightarrow \mathcal{C}_{S_{0}} \times \mathcal{C}_{S_{1}}
$$

induced by the anchor maps (4.2) is an equivalence of categories. A unital symmetric monoidal structure on a category $\mathcal{C}$ is a fibration $B_{\infty} \mathcal{C} \rightarrow \Gamma_{+}^{o}$ that satisfies the Segal condition and is equipped with an equivalence $B_{\infty} \mathcal{C}_{\mathrm{pt}} \cong \mathcal{C}$. The opposite unital symmetric monoidal structure on the opposite category $\mathcal{C}^{o}$ is given by $B_{\infty} \mathcal{C}^{o}=\left(B_{\infty} \mathcal{C}\right)_{\perp}^{o}$. A monoidal structure on a functor $\gamma: \mathcal{C} \rightarrow$ $\mathcal{C}^{\prime}$ between two categories $\mathcal{C}, \mathcal{C}^{\prime}$ equipped with unital symmetric monoidal structures $B_{\infty} \mathcal{C}, B_{\infty} \mathcal{C}^{\prime}$ is given by a functor $B_{\infty} \gamma: B_{\infty} \mathcal{C} \rightarrow B_{\infty} \mathcal{C}^{\prime}$, cartesian over $\Gamma_{+}^{o}$ and equipped with an isomorphism $B_{\infty} \gamma(\mathrm{pt}) \cong \gamma$. A co-lax symmetric monoidal structure on $\gamma$ is given by a functor $B_{\infty} \gamma: B_{\infty} \mathcal{C} \rightarrow B_{\infty} \mathcal{C}^{\prime}$ that is cartesian over the anchor maps, and a lax symmetric monoidal structure on $\gamma$ is a co-lax one on the opposite functor $\gamma^{o}$.

We note that this is quite parallel to Definition 2.15 and Definition 2.16, In fact, the standard simplicial circle $\Sigma: \Delta^{o} \rightarrow$ Sets is obtained by gluing together the two ends of the standard 1-simplex, $\Sigma([n])$ is finite for any $[n]$ and has the distinguished point given by glued ends, and the opposite functor $\Sigma^{o}: \Delta \rightarrow \Gamma_{+}^{o}$ sends the maps $s$ and $t$ in (2.3) to the maps (4.2) in $\Gamma_{+}^{o}$ (and more generally, a map $f$ is an anchor map in $\Delta$ if and only if $\Sigma^{o}(f)$ is an anchor map in $\Gamma_{+}^{o}$ ). Therefore for any unital symmetric monoidal structure $B_{\infty} \mathcal{C}$ on a category $\mathcal{C}$, the pullback $B \mathcal{C}=\Sigma^{o *} B_{\infty} \mathcal{C}$ is a unital monoidal structure. A symmetric monoidal structure on a functor restricts to a monoidal structure in the sense of Definition 2.15, and a lax or a co-lax one restricts to a corresponding structure in the sense of Definition 2.16, Just as in the non-symmetric case, if a functor $\gamma: \mathcal{C} \rightarrow \mathcal{C}^{\prime}$ admits a rightadjoint $\gamma^{\dagger}$, then a co-lax symmetric monoidal structure on $\gamma$ induces a lax one on $\gamma^{\dagger}$, and vice versa.

Explicitly, for any unital symmetric monoidal structure $B_{\infty} \mathcal{C}$, the anchor maps in $\Gamma_{+}$serve to provide identification $B_{\infty} \mathcal{C}_{S} \cong \mathcal{C}^{S}, S \in \Gamma$, thus anchoring the a priori arbitrary fibers $B_{\infty} \mathcal{C}_{S}$ of the fibration $B_{\infty} \mathcal{C}$ to our category $\mathcal{C}$. Structural maps then encode the structure: the tensor product functor $\mathcal{C} \times \mathcal{C} \rightarrow \mathcal{C}$ is induced by the unique structural map pt $\sqcup$ pt $\rightarrow$ pt, the unit object corresponds to the map $\emptyset \rightarrow \mathrm{pt}$, and various associativity and commutativity isomorphisms are packaged into the isomorphisms (1.3) for the fibration $B_{\infty} \mathcal{C} \rightarrow \Gamma_{+}$. This can be made into an explicit comparison theorem but we will not need it. 
Remark 4.2. The Segal condition of Definition 4.1 is the original condition introduced by Segal in [S] (although he worked with topological spaces and weak equivalences rather then categories and equivalences). The (obvious) generalization to 2-categories appeared slightly later and inherited the name.

The first example of a unital symmetric monoidal structure is given by cartesian products: if a category $\mathcal{C}$ has finite products, they define a unital symmetric monoidal structure $B_{\infty} \mathcal{C}$. Explicitly, objects of $B_{\infty} \mathcal{C}$ are pairs $\left\langle S, c_{.}\right\rangle$of a finite set $S \in \Gamma$ and a collection of objects $c_{s} \in \mathcal{C}, s \in S$. A morphism $\left\langle S^{\prime}, c_{.}^{\prime}\right\rangle \rightarrow\left\langle S, c_{\bullet}\right\rangle$ is a pair of a diagram (4.1) and a collection of maps $c_{f(s)}^{\prime} \rightarrow c_{s}, s \in \widetilde{S} \subset S$. Note that the embedding $i_{c}:$ pt $\rightarrow \mathcal{C}$ onto any object $c \in \mathcal{C}$ carries a unique co-lax unital symmetric monodial structure $B_{\infty} i_{c}$ that sends a set $S$ to the collection $c_{s}=c$ of copies of the object $c$, and a morphism $f$ to the collection of identity maps id $: c \rightarrow c$. One can also consider the transpose cofibration $\left(B_{\infty} \mathcal{C}\right)_{\perp} \rightarrow \Gamma_{+}$. It has the same objects, and a morphism $\left\langle S, c_{\bullet}\right\rangle \rightarrow\left\langle S^{\prime}, c_{.}^{\prime}\right\rangle$ is a diagram (4.1) and a collection of maps

$$
\prod_{s \in f^{-1}\left(s^{\prime}\right)} c_{s} \rightarrow c_{s^{\prime}}, \quad s^{\prime} \in S^{\prime},
$$

where the product is the cartesian product in $\mathcal{C}$. Dually, if $\mathcal{C}$ has finite coproducts, they also define a unital symmetric monoidal structure. In fact, a unital symmetric monoidal structure $B_{\infty} \mathcal{C}$ on a category $\mathcal{C}$ defines a unital symmetric monoidal structure $B_{\infty} \mathcal{C}^{o}=\left(B_{\infty} \mathcal{C}\right)_{\perp}^{o}$ on the opposite category $\mathcal{C}^{o}$, and if $\mathcal{C}$ has finite coproducts, $\mathcal{C}^{o}$ has finite products.

The description of the cartesian product monoidal structure in terms of the maps (4.3) may look slightly artificial but it has its uses. For example, for any commutative ring $k$, the category $k$-mod is equipped with a forgetful functor $k$-mod $\rightarrow$ Sets. One can then define a unital symmetric monoidal structure $B_{\infty} k$-mod as follows: objects are pairs $\left\langle S, c_{\bullet}\right\rangle, S \in \Gamma, c_{\bullet} \in k$-mod, and a morphism in the transpose cofibration $\left(B_{\infty} k \text {-mod }\right)_{\perp}$ is a diagram (4.1) and a collection of set-theoretic maps (4.3) that are $k$-linear in each argument. This condition is obviously closed under compositions, so we indeed obtain a well-defined category. The corresponding product on $k$-mod is the usual tensor product $-\otimes_{k}-$ (in fact, this is how the tensor product of vector spaces is defined in any good linear algebra textbook).

To extend this further, assume that $k$ is Noetherian. Then the category $\operatorname{Comm}(k)$ of finitely generated commutative $k$-algebra has finite coproducts, hence the coproduct monoidal structure $B_{\infty} \operatorname{Comm}(k)$, and the transpose cofibration $\left(B_{\infty} \operatorname{Comm}(k)\right)_{\perp} \rightarrow \Gamma_{+}$can be also described by imposing conditions on the maps (4.3). Moreover, the same procedure then constructs 
a unital symmetric monoidal structure on the category $\operatorname{Comm}(k)$-mod of Example 1.17 and on the forgetful functor $\varphi: \operatorname{Comm}(k)-\bmod \rightarrow \operatorname{Comm}(k)$. The product is given by $\langle A, M\rangle \otimes\left\langle A^{\prime}, M^{\prime}\right\rangle=\left\langle A \otimes_{k} A^{\prime}, M \otimes_{k} M^{\prime}\right\rangle$. The opposite categories $\operatorname{Comm}(k)^{o}, \operatorname{Comm}(k)-\bmod ^{o}$ and the opposite functor $\varphi^{o}$ then also carry unital symmetric monoidal structures, and moreover, the functor $B_{\infty} \varphi^{o}: B_{\infty} \operatorname{Comm}(k)-\bmod ^{o} \rightarrow B_{\infty} \operatorname{Comm}(k)^{o}$ is a fibration. Furthermore, the category $\operatorname{Sch}(k)$ has the unital symmetric monoidal structure given by cartesian products, the full embedding $j: \operatorname{Comm}(k)^{o} \rightarrow \operatorname{Sch}(k)$ carries an obvious unital symmetric monoidal structure $B_{\infty} j$, and the pushforward fibration $B_{\infty} \mathrm{QCoh}(\operatorname{Sch}(k))^{o}=\left(B_{\infty} j\right)_{*} B_{\infty} \operatorname{Comm}(k)$-mod $^{o}$ defines a unital symmetric monoidal structure on $\mathrm{QCoh}(\operatorname{Sch}(k))^{\circ}$, hence also on $\mathrm{QCoh}(\operatorname{Sch}(k))$, with the product

$$
\langle X, \mathcal{F}\rangle \otimes\left\langle X^{\prime}, \mathcal{F}^{\prime}\right\rangle=\left\langle X \times_{\text {Spec } k} X^{\prime}, \mathcal{F} \otimes_{k} \mathcal{F}^{\prime}\right\rangle .
$$

As in Example 1.17, we can use the same procedure to obtain a unital symmetric monoidal structure $B_{\infty}$ QCoh. $(\operatorname{Sch}(k))^{o}$ on QCoh. $(\operatorname{Sch}(k))^{o}$, and since the tensor product of $h$-flat complexes is $h$-flat, it induces a unital symmetric monoidal structure $B_{\infty} \mathrm{QCoh}^{\mathrm{b}}(\operatorname{Sch}(k))^{\circ}$ on the full subcategory QCoh. $(\operatorname{Sch}(k))^{o} \subset$ QCoh. $(\operatorname{Sch}(k))^{o}$. Localizing with respect to quasiisomorphisms then gives a unital symmetric monoidal structure $B_{\infty} \mathcal{D}(\operatorname{Sch}(k))^{o}$ on $\mathcal{D}(\operatorname{Sch}(k))^{o}$ and the opposite symmetric monoidal structure $B_{\infty} \mathcal{D}(\operatorname{Sch}(k))$ on $\mathcal{D}(\operatorname{Sch}(k))$, with the product given by the derived version of (4.4). The forgetful bifibrations $\mathcal{D}(\mathrm{S} \operatorname{ch}(k)) \rightarrow \mathrm{Sch}(k)^{o}, \mathcal{D}(\mathrm{Sch}(k))^{o} \rightarrow \mathrm{Sch}(k)$ carry unital symmetric monoidal structures. Moreover, for any $X \in \operatorname{Sch}(k)$, we can equip the embedding $i_{X}: \mathrm{pt} \rightarrow \mathrm{Sch}(k)$ with its unique co-lax unital symmetric monoidal structure $B_{\infty} i_{X}$, and then $B_{\infty} \mathcal{D}(X)^{o}=\left(B_{\infty} i_{X}\right)^{*} B_{\infty} \mathcal{D}(\operatorname{Sch}(k))$ provides the usual symmetric monoidal structure on $\mathcal{D}(X)^{o} \cong i_{X}^{*} \mathcal{D}(\operatorname{Sch}(k))^{o}$.

4.2 Morita 2-category. The first real-life example of a 2-category that is not manifestly strict is probably the Morita 2-category: its objects are algebras $A$ over a fixed commutative ring $k$, morphisms from $A_{0}$ to $A_{1}$ are $A_{0}$ - $A_{1}$-bimodules (that is, $A_{0}^{o} \otimes_{k} A_{1}$-modules), and the composition is given by the tensor product. To construct it in terms of Definition 2.5, one can follow the procedure described in [K3, Section 8]. Slightly more generally, we start with an arbitrary unital monoidal category $\mathcal{C}$, with the product $-\otimes-$ and the unit object $1 \in \mathcal{C}$.

Definition 4.3. A $\mathcal{C}$-enrichment of a 2-category $\mathcal{E}$ is a co-lax 2-functor $A: \mathcal{E}^{\tau} \rightarrow B \mathcal{C}^{o}$ (that is, a lax 2-functor from $\mathcal{E}$ to $B \mathcal{C}$ ). A $\mathcal{C}$-enrichment $A$ of 
a small category $I$ is a $\mathcal{C}$-enrichment of its simplicial replacement $\Delta I$, and for any small category $I^{\prime}$ and a functor $\gamma: I^{\prime} \rightarrow I$, the induced enrichement $\gamma^{*} A$ is the composition $A \circ \Delta \gamma: \Delta I^{\prime} \rightarrow B \mathcal{C}^{\circ}$. A morphism from a $\mathcal{C}$-enrichement $A_{0}$ to a $\mathcal{C}$-enrichment $A_{1}$ is a map $A_{0}^{o} \rightarrow A_{1}^{o}$ between the opposite functors.

Example 4.4. If $I=\mathrm{pt}$, then a $\mathcal{C}$-enrichment $A: \Delta \rightarrow B \mathcal{C}^{o}$ is the same thing as an associative unital algebra object in $\mathcal{C}$ - the object itself is $A([1]) \in B \mathcal{C}_{[1]}^{o} \cong \mathcal{C}^{o}$, and the morphisms (1.5) for the transition functors (2.4) and (2.5) define the product $A([1]) \otimes A([1]) \rightarrow A([1])$ and the unit embedding $1 \rightarrow A([1])$. Morphisms between $\mathcal{C}$-enrichments correspond to algebra maps (this is why they are defined by passing to the opposite functors).

Example 4.5. More generally, if $I=e(S)$ for a set $S$, then a $\mathcal{C}$-enrichment for $I$ is a small $\mathcal{C}$-enriched category with the set of objects $S$ in the usual sense: we have a morphism object $A\left(s, s^{\prime}\right) \in \mathcal{C}$ for any $s, s^{\prime} \in S$, the composition maps $A\left(s, s^{\prime}\right) \otimes A\left(s^{\prime}, s^{\prime \prime}\right) \rightarrow A\left(s, s^{\prime \prime}\right)$, and the identity maps $1 \rightarrow A(s, s)$.

Example 4.6. If $I=[1]$, then a $\mathcal{C}$-enrichment $A$ for $I$ defines algebra objects $A_{00}, A_{11}$ by restricting to $0,1 \in[1]$, and evaluating $A$ on the tautological object id $\in \Delta[1]$ of Example 2.1 gives an $A_{11}-A_{00}$-bimodule $A_{01}$.

Example 4.7. More generally, if $I=[n], n \geq 0$, then a $\mathcal{C}$-enrichment $A$ for $I$ can be visualized as an upper-triangular matrix $\left(A_{i j}\right), 0 \leq i \leq j \leq n$ of objects in $\mathcal{C}$. For any $0 \leq i \leq j \leq l \leq n$, we have the product map $A_{i j} \otimes A_{j l} \rightarrow A_{i l}$, and these maps are associative and unital in the appropriate sense.

For any small category $I, \mathcal{C}$-enrichments of $I$ form a full subcategory in $\operatorname{Fun}_{\Delta}\left(\Delta I, B \mathcal{C}^{o}\right)^{o}$, and since enrichments are functorial with respect to functors $\gamma: I^{\prime} \rightarrow I$, these categories form a fibration over Cat whose objects are pairs $\langle I, A\rangle$. We can consider its restriction to $\Delta \subset$ Cat, and denote by $\operatorname{Aug}(\mathcal{C})$ its reduction (2.7). It is not our Morita 2-category, and in fact, it is not a 2-category at all: it does not satisfy the Segal condition. Indeed, already for $[n]=2$, we have the product map $A_{01} \otimes A_{12} \rightarrow A_{02}$ of Example 4.7, but nothing insures that it factors through an isomorphism $A_{01} \otimes_{A_{11}} A_{12} \cong A_{02}$ (nor that the tensor product is even well-defined).

To correct for this, consider the functor $\kappa=\rho \circ \lambda: \Delta \rightarrow \Delta,[n] \mapsto[n]^{<}$, and the fibration $\kappa^{*} B \mathcal{C}^{o}$ with the projection $a^{*}: \kappa^{*} B \mathcal{C}^{o} \rightarrow B \mathcal{C}^{o}$ induced by the adjunction map Id $\rightarrow \kappa$. Define a module over a $\mathcal{C}$-enriched small category $\langle I, A\rangle$ as a functor $M: \Delta I \rightarrow \kappa^{*} B \mathcal{C}^{\circ}$ over $\Delta$, cartesian over 
all initial embeddings $s:[l] \rightarrow[n]$ and equipped with an isomorphism $a^{*} \circ M \cong A$. Then $\langle I, A\rangle$-modules form a category, and we denote the opposite category by $\langle I, A\rangle$-mod. A functor $\gamma: I^{\prime} \rightarrow I$ defines a functor $\gamma^{*}:\langle I, A\rangle$-mod $\rightarrow\left\langle I^{\prime}, \gamma^{*} A\right\rangle$-mod, and an object $i \in I_{[0]} \subset \Delta I$ defines a functor $\langle I, A\rangle-\bmod \rightarrow \mathcal{C}, M \mapsto M(i) \in\left(\kappa^{*} B \mathcal{C}^{o}\right)_{[0]}^{o} \cong \mathcal{C}$. Moreover, it has been proved in [K3, Lemma 8.7] that if $i \in I$ is an initial object, then this functor has a left-adjoint $\mathcal{C} \rightarrow\langle I, A\rangle$-mod sending an object $V \in \mathcal{C}$ to some $\langle I, A\rangle$-module $V_{i}$. Modules of the form $V_{i}$ are called representable.

Definition 4.8. A $\mathcal{C}$-enriched category $\langle[n], A\rangle,[n] \in \Delta$ is a cylinder if for any $0 \leq l \leq l^{\prime} \leq n$, the functor $t^{*}:\left\langle\left[l^{\prime}\right], t^{*} A\right\rangle$-mod $\rightarrow\left\langle[l], t^{*} A\right\rangle$-mod sends representable modules to representable modules.

Example 4.9. In the situation of Example 4.6, $\langle[1], A\rangle$ is a cylinder iff $A_{01}$ considered as an $A_{11}$-module is of the form $A_{01} \cong V \otimes A_{11}$ for some $V \in \mathcal{C}$.

Proposition 4.10. The full subcategory $\operatorname{Mor}(\mathcal{C}) \subset \operatorname{Aug}(\mathcal{C})$ spanned by cylinders is a 2-category in the sense of Definition 2.5.

Proof. This is a particular case of [K3, Proposition 8.20], with the following modifications. Firstly, cylinders of Definition 4.8 correspond to iterated polycylinders of [K3, Definition 8.16]. Secondly, [K3] encodes 2-categories by transpose cofibrations $\mathcal{C}_{\perp} \rightarrow \Delta^{o}$ rather than fibrations $\mathcal{C} \rightarrow \Delta$, see Remark 2.6, so the notation might be different in places. Thirdly, [K3] actually deals with a more general situation that combines Example 4.5 and Example 4.6, and produces the Morita 2-category of enriched small categories. To obtain our $\mathcal{M o r}(\mathcal{C})$, one has to take the full 2-subcategory spanned by algebras (that is, categories with a single object).

To see how cylinders of Definition 4.8 encode tensor products, consider the functor $\kappa_{b}: \Delta^{<} \rightarrow \Delta,[n] \mapsto[n]^{<>}$. It sends the initial object $o \in \Delta^{<}$ to $[1] \in \Delta$, we have $\left(\kappa_{b}^{*} B \mathcal{C}\right)_{o} \cong(B \mathcal{C})_{[1]} \cong \mathcal{C}$, and by Remark 1.6, the embedding $\mathcal{C} \cong\left(\kappa_{b}^{*} B \mathcal{C}\right)_{o} \subset \kappa_{b}^{*} B \mathcal{C}$ admits a right-adjoint $\zeta: \kappa_{b}^{*} B \mathcal{C} \rightarrow \mathcal{C}$. Note that for any $[n] \in \Delta^{<}$, the unique map $[n] \rightarrow[0]$ gives rise to a $\operatorname{map} \kappa_{b}([n]) \rightarrow \kappa_{b}([0]) \cong[2]$, and this promotes the functor $\kappa_{b}$ to a functor $\kappa_{\dagger}: \Delta^{<} \rightarrow \Delta /{ }^{\text {ld }}[2] \cong \Delta[2]$. Then for any $\mathcal{C}$-enrichment $A$ of the small category [2], we obtain a functor $A^{\dagger}=\zeta^{o} \circ A \circ \kappa_{\dagger}^{o}: \Delta^{<o} \rightarrow \mathcal{C}$. Its value at the empty ordinal $o \in \Delta^{<}$is $A_{02} \in \mathcal{C}$, and for any $[n] \in \Delta \subset \Delta^{<}$, we have

$$
A^{\dagger}([n]) \cong A_{01} \otimes A_{11}^{\otimes n} \otimes A_{12}
$$


If $A$ is a cylinder, then $A^{\dagger}$ extends to the category $\Delta_{+}^{o} \supset \Delta^{<o}$, so that $A^{\dagger} \cong \lambda^{o *} A_{+}^{\dagger} \cong \rho_{!}^{o} A_{+}^{\dagger}$ for some $A_{+}^{\dagger}: \Delta_{+}^{o} \rightarrow \mathcal{C}$, where the functor $\rho_{!}^{o} \cong \lambda^{o *}$ is left-adjoint to $\rho^{o *}$. Then since $o \in \Delta_{+}^{o}$ is terminal, we have

$$
A_{02} \cong A^{\dagger}(o) \cong \operatorname{colim}_{\Delta_{+}^{o}} A_{+}^{\dagger} \cong \operatorname{colim}_{\Delta^{o}} \rho_{!}^{o} A_{+}^{\dagger} \cong \operatorname{colim}_{\Delta^{o}} A^{\dagger},
$$

and in particular, the colimit exists. This colimit reduces to the coequalizer of the two maps $A^{\dagger}(s), A^{\dagger}(t): A_{01} \otimes A_{11} \otimes A_{12} \rightarrow A_{01} \otimes A_{12}$ that encode the two multiplications, and this is the usual definition of $A_{01} \otimes_{A_{11}} A_{12}$.

Example 4.11. For a somewhat tautological but very useful example of a Morita 2-category in the sense of Proposition 4.10, let $\mathcal{C}$ be a category with finite products, considered as a unital monoidal category with respect to these products. Then by definition, objects in $\mathcal{M o r}\left(\mathcal{C}^{o}\right)$ are objects $c \in \mathcal{C}$ equipped with a counital coalgebra structure, and just as in the symmetric monoidal case, there is exactly one such for any $c$, with the coproduct $c \rightarrow$ $c \times c$ given by the diagonal map. Thus $\mathcal{M o r}\left(\mathcal{C}^{\circ}\right)$ has the same objects as $\mathcal{C}$, and moreover, the morphism category $\operatorname{Mor}\left(\mathcal{C}^{o}\right)\left(c, c^{\prime}\right), c, c^{\prime} \in \mathcal{C}$ is opposite to the category of diagrams

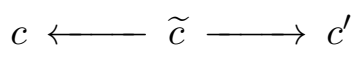

in $\mathcal{C}$ such that $\widetilde{c} \cong x \times c^{\prime}$ for some $x \in \mathcal{C}$ (this is the cylinder condition). Composition of the diagrams (4.6) is given by fibered products that exists by virtue of the cylinder condition. Informally, $\operatorname{Mor}\left(\mathcal{C}^{o}\right)$ is 2-opposite to the 2-category of correspondences in $\mathcal{C}$. Moreover, if we let $\bar{C}=\mathcal{C}_{\mathrm{ld}}$ be the discrete category underlying $\mathcal{C}$, and let $e(\mathcal{C})=e(\bar{C})$ be the category with the same objects as $\mathcal{C}$ and exactly one morphism between any two objects, then $\Delta e(\mathcal{C}) \cong \varepsilon_{*} \bar{C}$, where $\varepsilon:$ pt $\rightarrow \Delta$ is the embedding onto $[0] \in \Delta$, and the identification $\mathcal{M o r}\left(\mathcal{C}^{o}\right)_{[0]} \cong \bar{C}$ induces a 2-functor $\operatorname{Mor}\left(\mathcal{C}^{o}\right) \rightarrow \Delta e(\mathcal{C})$. A useful observation is that it admits a left-adjoint co-lax 2-functor

$$
i: \Delta e(\mathcal{C}) \rightarrow \operatorname{Mor}\left(\mathcal{C}^{o}\right) .
$$

It is identical over [0], and for any $c, c^{\prime} \in \mathcal{C}$, the unique map from $c$ to $c^{\prime}$ goes to the initial object of the category $\operatorname{Mor}\left(\mathcal{C}^{o}\right)\left(c, c^{\prime}\right)$ - namely, the diagram (4.6) with $\widetilde{c}=c \times c^{\prime}$.

For a useful generalization of Proposition 4.10, assume that the monoidal category $\mathcal{C}$ has all filtered colimits preserved by the tensor product. Then for any $\mathcal{C}$-enriched category $\langle I, A\rangle,\langle I, A\rangle$-mod also has filtered colimits, 
and one can define ind-representable modules as filtered colimits of representable ones. Define ind-cylinders by replacing "representable" with "indrepresentable" in Definition 4.8, and let $\mathcal{M o r}^{*}(\mathcal{C}) \subset \operatorname{Aug}(\mathcal{C})$ be the full subcategory spanned by ind-cylinders. Then [K3, Proposition 8.20] also applies to $\operatorname{Mor}^{*}(\mathcal{C})$, so that it is also a 2-category. It has the same objects as $\mathcal{M o r}(\mathcal{C})$ but more morphisms between them.

4.3 Derived Morita 2-category. For a real-life application of Proposition 4.10, take a commutative ring $k$, and let $\mathcal{C}=k$-mod ${ }^{f l}$, the category of flat $k$-modules. Then objects in $\mathcal{M o r}^{*}(k)=\mathcal{M o r}{ }^{*}\left(k-\bmod ^{f l}\right)$ are flat $k$ algebras, and by Example 4.9, morphisms from $A_{0}$ to $A_{1}$ in $\mathcal{M o r}(\mathcal{C})$ are given by $A_{0}$ - $A_{1}$-bimodules of the form $V \otimes A_{1}, V \in k$-mod ${ }^{f l}$. By the standard criterion of flatness, morphisms in $\mathcal{M o r}^{*}(\mathcal{C})$ are bimodules that are flat over $A_{1}$ ("right-flat"). Composition is given by the usual tensor product over $A_{1}$.

To generalize this to complexes, we need to generalize slightly Proposition 4.10. Assume given unital monoidal categories $\mathcal{C}, \mathcal{C}^{\prime}$, and a unital monoidal functor $\varphi: \mathcal{C} \rightarrow \mathcal{C}^{\prime}$. Say that $\langle[n], A\rangle \in \operatorname{Aug}(\mathcal{C})$ is a $\varphi$-cylinder resp. $\varphi$-ind-cylinder if $B \varphi \circ A$ is a cylinder resp. ind-cylinder in the sense of Definition 4.8, and let $\mathcal{M o r}(\mathcal{C}, \varphi), \mathcal{M o r}^{*}(\mathcal{C}, \varphi) \subset \operatorname{Aug}(\mathcal{C})$ be the full subcategories spanned by $\varphi$-cylinders resp. $\varphi$-ind-cylinders.

Lemma 4.12. Assume that the functor $\varphi: \mathcal{C} \rightarrow \mathcal{C}^{\prime}$ is conservative and creates colimits - that is, for any small $I$ and functor $c: I \rightarrow \mathcal{C}$, the colimit $\operatorname{colim}_{I} c$ exists if and only if so does $\operatorname{colim}_{I} \varphi(c)$, and in this case, $\operatorname{colim}_{I} \varphi(c) \cong \varphi\left(\operatorname{colim}_{I} c\right)$. Then $\mathcal{M o r}(\mathcal{C}, \varphi)$ is a 2-category, and $\operatorname{Aug}(\varphi)$ restricts to a 2-functor $\operatorname{Mor}(\mathcal{C}, \varphi) \rightarrow \mathcal{M o r}\left(\mathcal{C}^{\prime}\right)$. Moreover, if $\mathcal{C}^{\prime}$ has filtered colimits, then the same holds for $\mathcal{M o r}^{*}(\mathcal{C}, \varphi)$ and $\mathcal{M o r}^{*}\left(\mathcal{C}^{\prime}\right)$.

Proof. Proposition 4.10 is a part of [K3, Proposition 8.20] whose proof relies on [K3, Lemma 8.21] and [K3, Lemma 8.22]. Out of these, Lemma 8.21 holds for $\varphi$-cylinders and $\varphi$-ind-cylinders with the same proof, and so does Lemma 8.22 (i). For (ii) and (iii) of Lemma 8.22, notice that since $\varphi$ creates colimits, it suffices to check the existence of $\beta_{!}^{\Delta^{o}}$ after applying $\varphi$, and since $\varphi$ is also conservative, checking that $\beta_{!}^{\Delta^{o}} \beta^{*} A \rightarrow A$ is an isomorphism can also be done after applying $\varphi$. Finally, the proof of Proposition 8.20 itself for $\varphi$-cylinders and $\varphi$-ind-cylinders is again identically the same.

For applications, we take $\mathcal{C}=\mathcal{C}_{.}^{f l}(k)$, the category of termwise-flat chain complexes of $k$-modules, and let $\mathcal{C}^{\prime} \subset \mathcal{C}$ be the full subcategory spanned by complexes with zero differential. Then forgetting the differential gives 
a conservative functor $\varphi: \mathcal{C} \rightarrow \mathcal{C}^{\prime}$ that creates colimits, so we can form the 2-categories $\mathcal{M o r} .(k)=\operatorname{Mor}(\mathcal{C}, \varphi), \mathcal{M o r}^{*}(k)=\mathcal{M o r}^{*}(\mathcal{C}, \varphi)$. By definition, objects in these 2-categories are termwise-flat DG algebras $A$. over $k$. Morphisms from $A_{\text {. }}$ to $A_{\text {. }}^{\prime}$ in $\mathcal{M o r} .(k)$ resp. $\mathcal{M o r}_{.}^{*}(k)$ are $A_{\text {. }}^{o} \otimes_{k} A_{\text {.- }}^{\prime}$ modules $M$. that are semifree resp. semiflat over $A^{\prime}$. (that is, become free resp. flat if we forget the differential). We can then consider the subcategory $\mathcal{M o r}^{b}(k) \subset \mathcal{M o r}^{*}(k)$ whose objects are DG algebras that are pointwise-flat and $h$-flat as complexes of $k$-modules, and whose morphisms are $A_{\text {. }}^{o} \otimes_{k} A_{\text {.- }}$ modules that are semiflat and $h$-flat over $A^{\prime}$. Since the composition in $\mathcal{M o r}^{*}(k)$ is given by the usual tensor product, $\mathcal{M o r}^{b}(k) \subset \mathcal{M o r}^{*}(k)$ is a 2 -subcategory, and the transition functors of the fibration $\mathcal{M o r}^{b} .(k) \rightarrow \Delta$ send quasiisomorphisms to quasiisomorphisms. We can then invert those quasiisomorphisms and obtain the derived Morita 2-category $\mathcal{D} \mathcal{M}$ or $(k)$.

Remark 4.13. One usually interprets $\mathcal{D} \mathcal{M}$ or $(k)$ as the 2-category of DG algebras over $k$ and $k$-linear triangulated functors between their derived categories but there is a caveat. For any $h$-flat termwise-flat DG algebras $A_{\text {. }} A^{\prime}, B_{\text {. over }} k$, an object $M \in \mathcal{D} \mathcal{M}$ or $(k)\left(A_{\text {. }}, A_{\text {. }}^{\prime}\right)$ induces a functor

$$
\text { - oM: } \mathcal{D} \mathcal{M} o r(k)\left(B_{.}, A_{\bullet}\right) \rightarrow \mathcal{D} \mathcal{M} o r(k)\left(B_{.}, A_{\bullet}^{\prime}\right),
$$

and the collection of functors (4.8) determines $M$ (this is just the Yoneda embedding used in Theorem 2.12). If $B .=k$, then for any $A . \in \mathcal{D} \mathcal{M}$ or $(k)_{[0]}$, we have $\mathcal{D} \mathcal{M} o r(k)\left(k, A_{\text {. }}\right) \cong \mathcal{D}\left(A_{\text {. }}\right)$, so that (4.8) is a functor $\mathcal{D}(A$. $) \rightarrow \mathcal{D}\left(A^{\prime}\right)$ between the derived categories of DG modules. But by itself, this functor is not enough to recover $M$ - the correspondence $\mathcal{D} \mathcal{M} o r(k)\left(A_{.}, A_{.}^{\prime}\right) \rightarrow$ $\operatorname{Fun}\left(\mathcal{D}\left(A_{\bullet}\right), \mathcal{D}\left(A_{\text {. }}^{\prime}\right)\right)$ is neither faithful nor full, and one cannot describe its essential image in any reasonable way.

Although we will not need it, it is perhaps useful to describe $\mathcal{D} \mathcal{M}$ or $(k)$ in another way that is somewhat more homotopy-invariant. Denote by $C$. $(k)$ the category of all complexes of $k$-modules, and let $\operatorname{Aug} .(k) \subset \operatorname{Aug}(C$. $(k))$ be the full subcategory spanned by objects $\langle[n], A\rangle$ such that for any map $f$ : $[0] \rightarrow[n]$, the pullback $\left\langle[0], f^{*} A\right\rangle$ lies in $\mathcal{M o r}^{b}{ }^{\circ}(k)_{[0]}$ (in terms of Example 4.7, this means that for any $i \in[n]$, the DG algebra $A_{i i}$ is termwise-flat and $h$-flat over $k)$. For any $[n] \in \Delta$, say that a map $\alpha:\langle[n], A\rangle \rightarrow\left\langle[n], A^{\prime}\right\rangle$ in Aug. $(k)_{[n]}$ is a quasiisomorphism if so is its pullback $f^{*} \alpha$ with respect to any map $f:[1] \rightarrow[n]$ (in terms of Example 4.7, this means that $\alpha: A_{i j} \rightarrow A_{i j}^{\prime}$ is a quasiisomorphism for any $0 \leq i \leq j \leq n$ ). Then note that for any $\langle[2], A\rangle \in \operatorname{Aug} .(k)_{[2]}$, we have the functor $A^{\dagger}: \Delta^{<o} \rightarrow C .(k)$ of (4.5). This is an augmented simplicial complex of $k$-modules. We can then take its 
normalized chain complex in the usual way, and obtain a bicomplex $A_{\text {... }}^{\dagger}$ Say that $\langle[2], A\rangle$ is a quasicylinder if the sum-total complex $A_{\text {. }}^{\dagger}$ of the bicomplex $A_{\text {., }}^{\dagger}$ is acyclic, and more generally, say that any $\langle[n], A\rangle \in \operatorname{Aug}_{\text {. }}(k)_{[n]}$ is a quasicylinder if so is its pullback $\left\langle[2], f^{*} A\right\rangle$ for any map $f:[2] \rightarrow[n]$. By definition, the projection Aug. $(k) \rightarrow \Delta$ is a fibration whose transition functors send quasiisomorphisms to quasiisomorphisms and quasicylinders to quasicylinders.

Lemma 4.14. (i) For any $[n] \in \Delta$, the fiber Aug. $(k)_{[n]}$ of the fibration $\operatorname{Aug} .(k) \rightarrow \Delta$ admits a localization $\mathcal{D} \operatorname{Aug}(k)_{[n]}$ with respect to quasiisomorphisms, so that we have a fibration $\mathcal{D} \operatorname{Aug}(k) \rightarrow \Delta$ with fibers $\mathcal{D} \operatorname{Aug}(k)_{[n]}$ and transition functors induced by those of Aug. $(k)$.

(ii) The derived Morita 2-category $\mathcal{D} \mathcal{M}$ or $(k)$ is equivalent to the full subcategory in $\mathcal{D} \operatorname{Aug}(k)$ spanned by quasicylinders.

Proof. For (i), for any DG algebra $A$ termwise-flat and $h$-flat over $k$, let $T(A)$ be the category of DG algebras $A^{\prime}$ over $k$ equipped with an additional auxiliary non-negative grading $A^{\prime}$. and an isomorphism $A_{0}^{\prime} \cong A$. Recall that the category of DG algebras over $k$ carries a model structure whose fibrations are termwise-surjective maps and whose weak equivalences are quasiisomorphisms $([\mathrm{H}],[\mathrm{Ke}$, Section 4$])$, and note that the same holds for $T(A)$, with the same proof (to construct cofibrant replacements, note that the free algebra $T(M, n)$ spanned by a cofibrant $A^{o} \otimes_{k} A$-module $M$ placed in auxiliary degree $n \geq 1$ is cofibrant by adjunction, and construct a replacement for some $A^{\prime} \in T(A)$ by starting with $A$, and adding the necessary new generators in each auxiliary degree $n$ by induction on $n$ ). But for any $[n] \in \Delta$, we have a full embedding

$$
\operatorname{Aug.}(k)_{[n]} \subset \coprod T\left(A_{00} \oplus A_{11} \oplus \cdots \oplus A_{n n}\right), A_{00}, \ldots, A_{n n} \in \operatorname{Aug}_{.}(k)_{[0]}
$$

that sends $\langle[n], A\rangle$ to the sum of its components $A_{i j}$ of Example 4.7, with $A_{i j}$ placed in auxiliary degree $j-i$, and the model structure on its target restricts to a model structure on its source.

For (ii), note that the embedding $\mathcal{M o r}^{b}(k) \subset \operatorname{Aug} .(k)$ descends to a functor $\mathcal{D} \mathcal{M}$ or $(k) \rightarrow \mathcal{D} \operatorname{Aug}(k)$ cartesian over $\Delta$. Let $\mathcal{D} \mathcal{M}$ or $(k)^{\prime} \subset \mathcal{D} \operatorname{Aug}(k)$ be the full subcategory spanned by quasicylinders. Since the quasicylnder condition is preserved by pullbacks, $\mathcal{D} \mathcal{M}$ or $(k)^{\prime} \rightarrow \Delta$ is a fibration, and we have to check that for any $[n]$, the functor

$$
\gamma_{n}: \mathcal{D} \mathcal{M o r}(k)_{[n]} \rightarrow \mathcal{D} \operatorname{Aug}(k)_{[n]}
$$


factors through an equivalence $\mathcal{D} \mathcal{M}$ or $(k)_{[n]} \cong \mathcal{D} \mathcal{M}$ or $(k)_{[n]}^{\prime}$. For $n=0$, the statement is tautological, and for an $n=1$, the quasicylinder condition is empty, so it suffices to notice that for any termwise-flat $h$-flat DG algebras $A_{0}, A_{1}$ over $k$, any $A_{0}^{o} \otimes_{k} A_{1}$-module $M$ is quasiisomorphic to a module that is semiflat and $h$-flat over $A_{1}$. However, more is true: any $M$ is quasiisomorphic to a filtered colimit of iterated extensions of free modules $A_{0}^{o} \otimes_{k} V \otimes_{k} A_{1}, V \in k$-mod ${ }^{f l}$. Therefore for any $[n] \in \Delta$, any object in $\operatorname{Mor}^{b}(k)_{[n]}$ is quasiisomorphic to a filtered colimit of iterated extensions of cylinders. But for any cylinder $\langle[2], A\rangle$, the functor $A^{\dagger}$ extends to $\Delta_{+}^{o} \supset \Delta^{<o}$, and this means that the normalized bicomplex $A_{\text {.. }}^{\dagger}$ is contractible in one of the directions, so that its totalization is acyclic. Therefore the embedding (4.10) sends cylinders to quasicylinders, and since the quasicylinder condition is closed under extensions and filtered colimits, the whole (4.10) factors through $\mathcal{D} \mathcal{M}$ or $(k)_{[n]}^{\prime}$.

Now observe that for any termwise-flat $h$-flat DG algebra $A$ over $k$, we have the functor $T(A) \rightarrow\left(A^{o} \otimes_{k} A\right)$-mod sending a DG algebra $A^{\prime} \in T(A)$ to its component $A_{1}^{\prime}$ of auxiliary degree 1 , and this functor has a fully faithful left-adjoint $M \mapsto T(M, 1)$. This defines a Quillen adjunction, thus an adjunction of the localizations, and when we restrict it via the embeddings (4.9), we end up with an adjoint pair consisting of the embedding (4.10) and a functor $\gamma_{n}^{\dagger}$ right-adjoint to it. Therefore firstly, (4.10) is fully faithful, and secondly, its essential image consists of objects $A$ such that the adjunction map $a: \gamma_{n} \gamma_{n}^{\dagger} A \rightarrow A$ is an isomorphism in $\mathcal{D} \operatorname{Aug}(k)_{[n]}$.

To finish the proof, it suffices to show that this holds for any quasicylinder, and since $\gamma_{n}^{\dagger}(a)$ is an isomorphism by adjunction, it suffices to show that $\gamma_{n}^{\dagger}$ is conservative on $\mathcal{D} \mathcal{M} \operatorname{or}(k)_{[n]}^{\prime}$. For $n=2$, this is clear: if a morphism $\alpha: A \rightarrow A^{\prime}$ induces quasiisomorphisms $A_{01} \rightarrow A_{01}^{\prime}, A_{11} \rightarrow A_{11}^{\prime}$, $A_{12} \rightarrow A_{12}^{\prime}$, then it induces quasiisomorphisms on all the terms in (4.5), and since the total complexes are acyclic, the component $A_{02} \rightarrow A_{02}^{\prime}$ must also be a quasiisomorphism. The general case then follows by induction on $n$.

Remark 4.15. Note that the quasicylinder condition is manifestly symmetric. Therefore Lemma 4.14 immediately implies that we have an equivalence

$$
\iota: \mathcal{D} \mathcal{M} \text { or }(k) \cong \mathcal{D} \mathcal{M} \text { or }(k)^{\iota}
$$

sending a DG algebra $A$. to the opposite DG algebra $A^{o}$.

4.4 Fourier-Mukai 2-category. Let us now combine Example 4.11 with the technology of Subsection 4.3, and apply it to the categories of Exam- 
ple 1.17, with the monoidal structures of Subsection 4.1. Assume that $k$ is Noetherian. The category $\mathrm{Sch}(k)$ has finite products, so that Example 4.11 applies directly, and shows that the Morita 2-category $\operatorname{Mor}\left(\operatorname{Sch}(k)^{o}\right)$ is 2opposite to the 2-category of correspondences in $\operatorname{Sch}(k)$. Then as in Subsection 4.3, we let $\mathcal{C} \subset$ QCoh. $(\operatorname{Sch}(k))$ be the full subcategory spanned by pairs $\langle X, \mathcal{F}$. $\rangle$ such that the complex $\mathcal{F}$. of quasicoherent sheaves of $\mathcal{O}_{X}$-modules is termwise-flat, we consider the forgetfull functor $\varphi: \mathcal{C} \rightarrow \mathcal{C}^{\prime}$, where $\mathcal{C}^{\prime} \subset \mathcal{C}$ consists of complexes with trivial differential, and we construct the Morita 2-category $\operatorname{Mor}(\mathcal{C}, \varphi)$. Moreover, while $\mathcal{C}$ does not necessarily have all filtered colimits, the fibers of the cofibration $\mathcal{C} \rightarrow \operatorname{Sch}(k)^{o}$ do; we add those filtered colimits of $\varphi$-cylinders to $\operatorname{Mor}(\mathcal{C}, \varphi)$ and by abuse of notation, denote the resulting 2-category by $\mathcal{M o r}^{*}(\mathcal{C}, \varphi)$. Then by definition, objects in $\mathcal{M o r}^{*}(\mathcal{C}, \varphi)$ are pairs $\langle X, \mathcal{A}$. $\rangle$ of a scheme $X \in \operatorname{Sch}(k)$ and a DG algebra $\mathcal{A}$. of flat quasicoherent sheaves on $X$, and morphisms from $\langle X, \mathcal{A}$. $\rangle$ to $\left\langle X^{\prime}, \mathcal{A}^{\prime}\right.$. $\rangle$ are pairs $\left\langle Z, \mathcal{M}_{\text {. }}\right\rangle$ of a correspondence $Z \rightarrow X \times X^{\prime}$ in $\operatorname{Sch}(k)$ abstractly isomorphic over $X^{\prime}$ to $Y \times X^{\prime}$ for some $Y \in \mathrm{Sch}(k)$, and a sheaf of DG $\pi^{*} \mathcal{A}_{\text {. }}^{o} \otimes_{\mathcal{O}_{Z}} \pi^{\prime *} \mathcal{A}_{\text {. }}^{\prime}$-modules on $Z$, semiflat over $\pi^{\prime *} \mathcal{A}_{\text {. }}^{\prime}$, where $\pi: Z \rightarrow X$, $\pi^{\prime}: Z \rightarrow Z$ are the projections. If we have another object $\left\langle X^{\prime \prime}, \mathcal{A}_{.}^{\prime \prime}\right\rangle$ and a morphism $\left\langle Z^{\prime}, \mathcal{M}_{\text {. }}^{\prime}\right\rangle$ from $\left\langle X^{\prime}, \mathcal{A}_{\text {. }}^{\prime}\right\rangle$ to $\left\langle X^{\prime \prime}, \mathcal{A}_{\text {. }}^{\prime \prime}\right\rangle$, then the composition is given by

$$
\left\langle Z, \mathcal{M}_{\bullet}\right\rangle \circ\left\langle Z^{\prime}, \mathcal{M}_{\bullet}^{\prime}\right\rangle \cong\left\langle Z \times_{X^{\prime}} Z^{\prime}, \nu^{*} \mathcal{M}_{\bullet} \otimes_{\eta^{*}} \mathcal{A}_{\bullet} \nu^{\prime *} \mathcal{M}_{\bullet}^{\prime}\right\rangle
$$

where $\nu: Z \times_{X^{\prime}} Z^{\prime} \rightarrow Z, \nu^{\prime}: Z \times_{X^{\prime}} Z^{\prime} \rightarrow Z^{\prime}, \eta=\pi^{\prime} \circ \nu: Z \times_{X^{\prime}} Z^{\prime} \rightarrow X^{\prime}$ are again the projections.

We then observe that by virtue of (4.12), objects $\left\langle X, \mathcal{A}_{\text {. }}\right\rangle$ such that $\mathcal{A}$. is $h$-flat over $X$, and morphisms $\langle Z, \mathcal{M}$. $\rangle$ such that $\mathcal{M}$. is $h$-flat over $Z$ form a 2-subcategory $\mathcal{M}$ or $^{\mathrm{b}} .(\operatorname{Sch}(k)) \subset \mathcal{M} \operatorname{cr}^{*}(\mathcal{C}, \varphi)$. We then have a pair of 2-opposite 2-functors

$$
\begin{gathered}
\psi: \mathcal{M}{ }^{b} .(\operatorname{Sch}(k)) \rightarrow \mathcal{M} \operatorname{or}\left(\operatorname{Sch}(k)^{o}\right), \\
\psi^{\tau}: \mathcal{M o r}^{b}(\operatorname{Sch}(k))^{\tau} \rightarrow \mathcal{M} \operatorname{Mor}\left(\operatorname{Sch}(k)^{o}\right)^{\tau},
\end{gathered}
$$

where $\psi$ is induced by the forgetful functor QCoh. $(\operatorname{Sch}(k)) \rightarrow \operatorname{Sch}(k)^{o}$.

Lemma 4.16. The functor $\psi^{\tau}$ of (4.13) is a fibration.

Proof. Use Lemma 1.11, The conditions (i), (ii) hold by definition. For (iii), note that $\psi^{\tau}$ is an identity over $[0] \in \Delta$, and its fiber over some $Z \rightarrow X \times X^{\prime}$ in $\operatorname{Mor}\left(\operatorname{Sch}(k)^{o}\right)_{[1]}^{\tau}$ is given by

$$
\mathcal{M o r}_{\bullet}^{b}(\operatorname{Sch}(k))_{Z}^{\tau} \cong \coprod_{\mathcal{A}_{\bullet}, \mathcal{A}_{\bullet}^{\prime}} \operatorname{QCoh}_{\bullet}^{b}\left(Z, \pi^{*} \mathcal{A}_{\bullet}^{o} \otimes_{\mathcal{O}_{Z}} \pi^{\prime *} \mathcal{A}_{\bullet}^{\prime}\right)^{o}
$$


where the disjoint union is over all pairs of $h$-flat termwise-flat DG algebras $\mathcal{A}_{\text {. }} \in \mathrm{QCoh} .(X), \mathcal{A}_{\text {. }}^{\prime} \in \mathrm{QCoh} .\left(X^{\prime}\right)$, and $\mathrm{QCoh}_{\text {. }}^{\mathrm{b}}(-,-)$ stands for the category of DG modules $h$-flat over $\mathcal{O}_{Z}$ and semiflat over $\pi^{\prime *} \mathcal{A}_{.}^{\prime}$. Then $\psi^{\tau}([1])$ is obviously a fibration, with the transition functor

$$
f^{*}\left(\left\langle Z, \mathcal{M}_{\bullet}\right\rangle\right)=\left\langle W, f^{*} \mathcal{M}_{\bullet}\right\rangle
$$

for any map $f: W \rightarrow Z$ of correspondences from $X$ and $X^{\prime}$, and by the Segal condition, $\psi^{\tau}([n])$ is a fibration for any $[n]$. Then again by the Segal condition, it suffices to check (iv) for the map $m:[1] \rightarrow[2]$ in $\Delta$ of (2.4), and in this case, it immediately follows from (4.14) and (4.12).

Now note that the transition functors (4.14) of the fibration $\psi^{\tau}$ provided by Lemma 4.16 obviously preserve quasiisomorphisms. Therefore we can again localize with respect to quasiisomorphims and obtain a category $\mathcal{E}$ fibered over $\operatorname{Mor}\left(\operatorname{Sch}(k)^{o}\right)^{\tau}$, with the fiber over some $Z \rightarrow X \times X^{\prime}$ given by the disjoint union of the derived categories $\mathcal{D}\left(Z, \pi^{*} \mathcal{A} . \otimes_{\mathcal{O}_{X}} \pi^{\prime *} \mathcal{A}^{\prime}\right.$.). The category $\mathcal{E}$ satisfies the Segal condition, and defines a 2-category in the sense of Definition 2.5. We denote the 2-opposite 2-category by $\mathcal{D} \mathcal{M} \operatorname{or}(\operatorname{Sch}(k))$ and call it the big derived Morita 2-category over $k$. The functor $\psi$ of (4.13) then induces a 2-functor

$$
\psi: \mathcal{D} \mathcal{M} \text { or }(\operatorname{Sch}(k)) \rightarrow \mathcal{M} \text { or }\left(\operatorname{Sch}(k)^{o}\right) .
$$

Objects of $\mathcal{D} \mathcal{M} \operatorname{or}(\operatorname{Sch}(k))$ are again pairs $\left\langle X, \mathcal{A}_{\mathbf{.}}\right\rangle$, and morphisms are pairs $\langle Z, \mathcal{M}\rangle, \mathcal{M} \in \mathcal{D}\left(Z, \pi^{*} \mathcal{A}_{\text {. }}^{o} \otimes_{\mathcal{O}_{Z}} \pi^{\prime *} \mathcal{A}_{\text {. }}^{\prime}\right)$, where $Z \cong Y \times X^{\prime}$ for some $Y$. Composition is given by the derived version of (4.12).

Lemma 4.17. The 2-functor (4.15) is a fibration.

Proof. As in Lemma 4.16, the conditions (i), (ii) of Lemma 1.11 are satisfied by definition, and it suffices to check (iii) over $[1] \in \Delta$ where it amounts to observing that when we pass to the derived categories, the transition functor (4.14) admits a right-adjoint $f_{*}$. Moreover, it suffices to check (iv) for the map $m$ of (2.4). To do this, assume given objects $\langle X, \mathcal{A}\rangle,.\left\langle X^{\prime}, \mathcal{A}_{\text {. }}^{\prime}\right\rangle$, $\left\langle X^{\prime \prime}, \mathcal{A}_{\text {. }}^{\prime \prime}\right\rangle$ in $\mathcal{D} \mathcal{M} \operatorname{or}(\operatorname{Sch}(k))_{[0]}$ and morphisms

$$
\begin{aligned}
f:\left\langle W, \mathcal{M}_{.}\right\rangle \rightarrow\left\langle Z, f_{*} \mathcal{M}_{\bullet}\right\rangle & \in \mathcal{D} \mathcal{M} \operatorname{or}(\operatorname{Sch}(k))\left(\left\langle X, \mathcal{A}_{\bullet}\right\rangle,\left\langle X^{\prime}, \mathcal{A}_{\bullet}^{\prime}\right\rangle\right), \\
f^{\prime}:\left\langle W^{\prime}, \mathcal{M}_{.}^{\prime}\right\rangle \rightarrow\left\langle Z^{\prime}, f_{*}^{\prime} \mathcal{M}_{.}^{\prime}\right\rangle & \in \mathcal{D} \mathcal{M} \operatorname{or}(\operatorname{Sch}(k))\left(\left\langle X^{\prime}, \mathcal{A}_{\bullet}^{\prime}\right\rangle,\left\langle X^{\prime \prime}, \mathcal{A}_{\bullet}^{\prime \prime}\right\rangle\right),
\end{aligned}
$$

both cartesian over $\mathcal{M}$ or $\left(\operatorname{Sch}(k)^{o}\right)_{[1]}$. Let $\nu, \nu^{\prime}$ and $\eta$ have the same meaning as in (4.12), and denote by $\varepsilon: W \times_{X^{\prime}} W^{\prime} \rightarrow W, \varepsilon^{\prime}: W \times_{X^{\prime}} W^{\prime} \rightarrow W^{\prime}$ and 
$\chi=\nu \circ\left(f \times f^{\prime}\right): W \times \times_{X^{\prime}} W \rightarrow X^{\prime}$ the corresponding projections for $W$ and for $W^{\prime}$. By (4.12), we then have to check that the base change map

$$
\nu^{*} f_{*} \mathcal{M} . \stackrel{\llcorner}{\otimes}_{\eta^{*} \mathcal{A}_{\bullet}^{\prime}} \nu^{\prime *} f_{*}^{\prime} \mathcal{M}_{\bullet}^{\prime} \rightarrow\left(f \times f^{\prime}\right)_{*} \varepsilon^{*} \mathcal{M} \cdot \stackrel{\llcorner}{\otimes}_{\chi^{*} \mathcal{A}_{\bullet}^{\prime}} \varepsilon^{\varepsilon^{*}} \mathcal{M}^{\prime}
$$

is an isomorphism. The pushforward functors are triangulated, and since we are working with schemes of finite type, they also commute with arbitrary sums. Therefore we may assume that $\mathcal{M}$. is free over $\chi^{*} \mathcal{A}^{\prime}$. - that is, we have $\mathcal{M}_{\mathbf{0}} \cong \mathcal{F}$. ${ }^{\llcorner} \mathcal{O}_{W} \chi^{\prime *} \mathcal{A}^{\prime}$. for some $\mathcal{F} . \in \mathcal{D}(W)$. Then by the projection formula, we have $f_{*} \mathcal{M}_{\bullet} \cong f_{*} \mathcal{F} . \stackrel{\llcorner}{\otimes}_{\mathcal{O}_{Z}} \nu^{\prime *} \mathcal{A}_{\bullet}^{\prime}$, and (4.16) can be rewritten as

$$
\nu^{*} f_{*} \mathcal{F} . \stackrel{\llcorner}{\otimes_{\mathcal{O}_{Z \times} X^{\prime} Z}} \nu^{\prime *} f_{*}^{\prime} \mathcal{M}_{.}^{\prime} \rightarrow\left(f \times f^{\prime}\right)_{*} \varepsilon^{*} \mathcal{M} . \stackrel{\llcorner}{\otimes}_{\mathcal{O}_{W \times_{X^{\prime}} W^{\prime}}} \varepsilon^{\prime *} \mathcal{M}_{\bullet}^{\prime} .
$$

Moreover, we have $\left(f \times f^{\prime}\right)=(f \times$ id $) \circ\left(\right.$ id $\left.\times f^{\prime}\right)$, so it suffices to consider separately the cases $f=$ id and $f^{\prime}=$ id. In the first case, we may again apply the projection formula to reduce to the case $\mathcal{F}$. $\cong \mathcal{O}_{W}$, and then (4.17) becomes the base change map $\nu^{*} f_{*} \mathcal{M}^{\prime}$. $\rightarrow(f \times \text { id })_{*} \varepsilon^{*} \mathcal{M}^{\prime}$. associated to the cartesian square

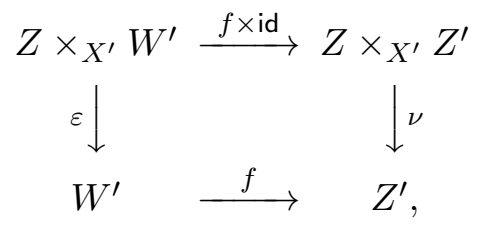

and since both $W$ and $Z$ are cylinders, the vertical maps are flat, so we are done by flat base change. In the second case, we reduce to the case $\mathcal{M}^{\prime} \cong \mathcal{O}_{W^{\prime}}$, and we note that while the vertical maps in the corresponding version of the cartesian square (4.18) are not flat, they become flat if we replace the products over $X^{\prime}$ with the absolute products. Then it suffices to check that the leftmost square in the diagram

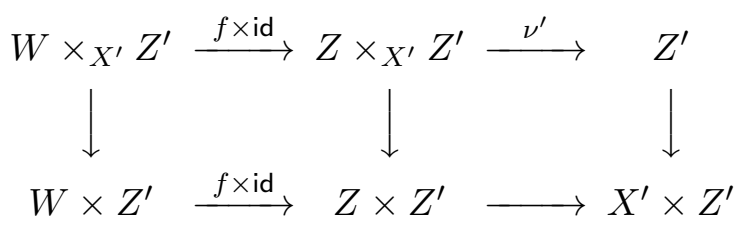

satisfies base change. But both squares are cartesian, and both maps $W \times$ $Z^{\prime}, Z \times Z^{\prime} \rightarrow X^{\prime} \times Z^{\prime}$ are flat, so we are again done by flat base change.

Now consider the co-lax 2-functor (4.7) for 2-the category $\mathcal{M}$ or $\left(\operatorname{Sch}(k)^{o}\right)$, and note that by virtue of Lemma 4.17, $\mathcal{F} \mathcal{M}^{\text {big }}(k)=i^{*} \mathcal{D} \mathcal{M} \operatorname{or}(\operatorname{Sch}(k))$ 
comes equipped with a fibration $\mathcal{F M}^{b i g}(k) \rightarrow \Delta e(\operatorname{Sch}(k)) \rightarrow \Delta$ that turns it into a 2-category in the sense of Definition 2.5. We call it the big FourierMukai 2-category over $k$. Objects are again pairs $\langle X, \mathcal{A}$. $\rangle$ of a scheme $X$ and an $h$-flat termwise-flat DG algebra $\mathcal{A}$., and the categories of morphisms are

$$
\mathcal{F} \mathcal{M}^{b i g}(k)\left(\left\langle X, \mathcal{A}_{\bullet}\right\rangle,\left\langle X^{\prime}, \mathcal{A}_{\bullet}^{\prime}\right\rangle\right) \cong \mathcal{D}\left(X \times X^{\prime}, \pi^{*} \mathcal{A}_{\bullet}^{o} \otimes_{\mathcal{O}_{X \times X^{\prime}}} \pi^{\prime *} \mathcal{A}_{\bullet}^{\prime}\right),
$$

where $\pi: X \times X^{\prime} \rightarrow X, \pi^{\prime}: X \times X^{\prime} \rightarrow X^{\prime}$ are the two projections. The composition $\mathcal{M}$. $\circ \mathcal{M}^{\prime}$. of morphisms $\mathcal{M} . \in \mathcal{F} \mathcal{M}^{b i g}\left(\left\langle X, \mathcal{A}_{.}\right\rangle,\left\langle X^{\prime}, \mathcal{A}^{\prime}\right\rangle\right)$ and $\mathcal{M}_{.}^{\prime} \in \mathcal{F} \mathcal{M}^{b i g}\left(\left\langle X^{\prime}, \mathcal{A}_{\bullet}^{\prime}\right\rangle,\left\langle X^{\prime \prime}, \mathcal{A}_{.}^{\prime \prime}\right\rangle\right)$ is given by

$$
\mathcal{M} . \circ \mathcal{M}_{.}^{\prime} \cong \rho_{*}\left(\nu^{*} \mathcal{M} . \stackrel{L}{\otimes}_{\eta^{*} \mathcal{A}_{.}^{\prime}} \nu^{\prime *} \mathcal{M}_{.}^{\prime}\right),
$$

where $\nu, \nu^{\prime}$ and $\eta$ are as in (4.12), and $\rho: X \times X^{\prime} \times X^{\prime \prime} \rightarrow X \times X^{\prime \prime}$ is the projection. We note that we have an obvious full embedding $\mathcal{D} \mathcal{M}$ or $(k) \subset$ $\mathcal{F} \mathcal{M}^{b i g}(k)$ that identifies $\mathcal{D} \mathcal{M}$ or $(k)$ with the 2-subcategory in $\mathcal{F} \mathcal{M}^{\text {big }}(k)$ spanned by pairs $\langle X, \mathcal{A}$. $\rangle$ with $X=\mathrm{pt}=\operatorname{Spec} k$. We also have the FourierMukai 2-category defined as the full 2-subcategory $\mathcal{F M}(k) \subset \mathcal{F} \mathcal{M}^{\text {big }}(k)$ spanned by pairs $\left\langle X, \mathcal{O}_{X}\right\rangle$. This is the same Fourier-Mukai 2-category as the one discussed in [AL2, Subsection 2.6]. We note that as in Remark 4.15, (4.19) is manifestly symmetric, so that we have an equivalence

$$
\iota: \mathcal{F} \mathcal{M}^{b i g}(k) \cong \mathcal{F} \mathcal{M}^{b i g}(k)^{\iota}
$$

sending $\left\langle X, \mathcal{A}_{\text {. }}\right\rangle$ to $\left.X, \mathcal{A}_{\text {. }}^{o}\right\rangle$.

4.5 Equivalences. According to our definition, two quasiisomorphic DG algebras over $k$ are different as objects in the derived Morita 2-category $\mathcal{D} \operatorname{Mor}(k)$. However, they are equivalent in the general 2-categorical sense. More precisely, assume given a map $f: A$. $\rightarrow B$. between $h$-flat termwiseflat DG algebras $A_{\text {. }}, B$. . Define the $g r a p h \operatorname{gr}(f)$ of the map $f$ as the object

$$
\operatorname{gr}(f)=A_{\bullet} \stackrel{\llcorner}{\otimes}_{A_{\bullet}^{o} \otimes_{k} A} A_{\bullet}^{o} \otimes_{k} B . \in \mathcal{D}\left(A_{\bullet}^{o} \otimes_{k} B_{\bullet}\right) \cong \mathcal{D} \mathcal{M} \text { or }(k)\left(A_{\bullet}, B_{\bullet}\right),
$$

where $A_{\text {. }}$ on the left is the diagonal bimodule, and $A_{\bullet}^{o} \otimes_{k} A_{\text {. acts on }} A_{\text {. }}^{o} \otimes_{k} B$. via the map id $\otimes f$. Then we have $\operatorname{gr}(f \circ g) \cong \operatorname{gr}(f) \circ \operatorname{gr}(g)$ for any composable pair of maps $f, g$, and if $f$ is a quasiisomorphism, we have

$$
\begin{aligned}
& \operatorname{gr}(f) \circ \iota\left(\operatorname{gr}\left(f^{o}\right)\right) \cong B_{.} \in \mathcal{D}\left(B_{.}^{o} \otimes_{k} B_{.}\right), \\
& \iota\left(\operatorname{gr}\left(f^{o}\right)\right) \circ \operatorname{gr}(f) \cong A_{.} \in \mathcal{D}\left(A_{.}^{o} \otimes_{k} A_{\bullet}\right),
\end{aligned}
$$

where $\iota$ is the equivalence (4.11) of Remark 4.15, Therefore $\operatorname{gr}(f)$ and $\iota\left(\operatorname{gr}\left(f^{o}\right)\right)$ are a pair of mutually inverse equivalences. Not all the equivalences 
in $\mathcal{D} \mathcal{M}$ or $(k)$ are of this form, but those that are can be easily characterized. Namely, both $A$. and $B$. define algebra objects $A, B$ in the monoidal category $\mathcal{D}(k)$, and any object $M_{\bullet} \in \mathcal{D}\left(A^{o} \otimes_{k} B_{\text {. }}\right)$ defines an object $M \in \mathcal{D}(k)$ that is a left module over $B$ and a right module over $A$. In particular, any map $e: k \rightarrow M$ defines action maps

$$
L(k): A=A \otimes k \rightarrow M, \quad R(k): B=B \otimes k \rightarrow M
$$

in the derived category $\mathcal{D}(k)$.

Lemma 4.18. An object $M_{.} \in \mathcal{D}\left(A_{.}^{o} \otimes_{k} B\right.$. $)$ is of the form $\operatorname{gr}(f)$ for a quasiisomorphism $f: A$. $\rightarrow B$. if and only if for some map $e: k \rightarrow M$ in $\mathcal{D}(k)$, both maps (4.24) are isomorphisms.

Proof. The "only if" part is immediate from (4.22) (since it is obvious for the diagonal bimodule). For the converse, represent $M$ by an $h$-projective $A_{\text {. }}^{o} \otimes_{k} B_{\text {.-module }} M_{\text {. }}$, lift $e$ to a map of complexes $e: k \rightarrow M_{\bullet}$, and note that it induces a quasiisomorphism $B_{\bullet}=B_{.} \otimes_{k} k \rightarrow M_{\text {. of }} B$.-modules. Then the action of $A$. and evaluation at $e(1) \in M_{0}$ give maps of complexes

$$
A_{\bullet}^{o} \stackrel{f}{\longrightarrow} \operatorname{End}_{B_{\bullet}^{*}}\left(M_{\bullet}\right)^{o} \stackrel{\text { ev }}{\longrightarrow} M_{\bullet},
$$

the map $f$ is a DG algebra map, and since $M$. is $h$-projective, the target of the map $f$ is quasiisomorphic to $B$. Then $M \cong \operatorname{gr}(f)$, and since ev and ev $\circ f$ in (4.25) are quasiisomorphisms by assumption, so is the map $f$.

Now let us turn to the Fourier-Mukai 2-category $\mathcal{F} \mathcal{M}^{\text {big }}(k)$. Recall that an object $\mathcal{E} \in \mathcal{C}$ in a unital monoidal category $\mathcal{C}$ is (left-)dualizable if it is reflexive as a morphism in the 2-category $B \mathcal{C}$; in this case, we have the adjoint morphism $\mathcal{E}^{\vee} \in(B \mathcal{C})_{[1]} \cong \mathcal{C}$ and the adjunction maps $\mathcal{E} \circ \mathcal{E}^{\vee} \rightarrow 1$, $1 \rightarrow \mathcal{E}^{\vee} \circ \mathcal{E}$. Recall also that for any $X \in \operatorname{Sch}(k)$, an object $\mathcal{E} \in \mathcal{D}(X)$ is dualizable if and only if it is perfect (that is, $\operatorname{Hom}(\mathcal{E},-)$ commutes with arbitrary sums). Finally, recall that $\mathcal{E} \in \mathcal{D}(X)$ is a generator if $\operatorname{Hom}(\mathcal{E}, \mathcal{F})=$ 0 implies $\mathcal{F}=0$, and recall that by $[\mathrm{BV}]$, any $X \in \operatorname{Sch}(X)$ admits a perfect generator $\mathcal{E} \in \mathcal{D}(X)$.

For any $\mathcal{E} \in \mathcal{D}(X)$, we can represent $\mathcal{E}$ by an $h$-injective complex $\mathcal{E}$. of quasicoherent sheaves on $X$, and then choose a cofibrant $h$-flat termwise-flat replacement $A(\mathcal{E}) . \rightarrow \operatorname{End}^{\bullet}(\mathcal{E}$. $)$ of the DG algebra of endomorphisms of the complex $\mathcal{E}_{\text {. }}$ Then $A(E)$. acts on $\mathcal{E}$., so we can promote $E$ to an object

$$
\mathcal{K}(E) \in \mathcal{D}\left(X, A(\mathcal{E}) . \otimes_{k} \mathcal{O}_{X}\right)=\mathcal{F} \mathcal{M}^{b i g}(k)\left(\left\langle X, \mathcal{O}_{X}\right\rangle,\langle\mathrm{pt}, A(\mathcal{E}) .\rangle\right)
$$


If $\mathcal{E}$ is perfect, thus dualizable, we have the dual object $\mathcal{E}^{\vee}$, and we can do the same procedure to obtain an $h$-flat termwise-flat DG algebra $A\left(E^{\vee}\right)$. and a object

$$
\mathcal{K}\left(\mathcal{E}^{\vee}\right) \in \mathcal{D}\left(X, A\left(\mathcal{E}^{\vee}\right) . \otimes_{k} \mathcal{O}_{X}\right)=\mathcal{D} \mathcal{M} \text { or }(k)\left(\left\langle\mathrm{pt}, A\left(\mathcal{E}^{\vee}\right)^{o}\right\rangle,\left\langle X, \mathcal{O}_{X}\right\rangle\right)
$$

where we use the equivalence (4.21) to interpret $\mathcal{K}\left(\mathcal{E}^{\vee}\right)$ as a morphism to and not from $\left\langle X, \mathcal{O}_{X}\right\rangle \cong\left\langle X, \mathcal{O}_{X}^{o}\right\rangle$.

Lemma 4.19. For any $X \in \operatorname{Sch}(h)$ and perfect $E \in \mathcal{D}(X)$, the objects (4.26) and (4.27) satisfy $\mathcal{K}(\mathcal{E}) \circ \mathcal{K}\left(\mathcal{E}^{\vee}\right) \cong$ id. If $\mathcal{E} \in \mathcal{D}(X)$ is a generator, then also $\mathcal{K}\left(\mathcal{E}^{\vee}\right) \circ \mathcal{K}(\mathcal{E}) \cong \mathrm{id}$.

Proof. For the first claim, note by (4.20), the object $M \in \mathcal{D}(k)$ underlying $\mathcal{K}(\mathcal{E}) \circ \mathcal{K}\left(\mathcal{E}^{\vee}\right) \in \mathcal{D}\left(A\left(\mathcal{E}^{\vee}\right)\right.$. $\otimes_{k} A(\mathcal{E})$. $)$ is given by

$$
M \cong H^{\bullet}\left(X, \mathcal{E} \stackrel{\llcorner}{\otimes}_{\mathcal{O}_{X}} \mathcal{E}^{\vee}\right),
$$

the algebras $\operatorname{End}^{\bullet}(\mathcal{E})$ resp. End ${ }^{\bullet}\left(\mathcal{E}^{\vee}\right)$ act on $M$ via $\mathcal{E}$ resp. $\mathcal{E}^{\vee}$, and then the adjunction map $\mathcal{O}_{X} \rightarrow \mathcal{E} \stackrel{\llcorner}{\otimes}_{\mathcal{O}_{X}} \mathcal{E}^{\vee}$ induces a map $e: k \rightarrow M$ such that both $L(k):$ End $^{\bullet}(\mathcal{E}) \rightarrow M$ and $R(k):$ End $^{\bullet}\left(\mathcal{E}^{\vee}\right) \rightarrow M$ are isomorphisms. We are then done by Lemma 4.18, and as a bonus, we obtain a quasiisomorphism between $A\left(\mathcal{E}^{\vee}\right)^{o}$. and $A(\mathcal{E})$.

For the second claim, note that composition with $\mathcal{K}(E)$ gives a functor

$$
\begin{aligned}
L(\mathcal{E}): \mathcal{D}\left(A(\mathcal{E})^{o}\right) \cong & \mathcal{F} \mathcal{M}^{b i g}(\langle\mathrm{pt}, A(\mathcal{E}) .\rangle,\langle\mathrm{pt}, k\rangle) \longrightarrow \\
& \longrightarrow \mathcal{F} \mathcal{M}^{b i g}\left(\left\langle X, \mathcal{O}_{X}\right\rangle,\langle\mathrm{pt}, k\rangle\right) \cong \mathcal{D}(X),
\end{aligned}
$$

and this functor has a right-adjoint $R(\mathcal{E}): \mathcal{D}(X) \rightarrow \mathcal{D}\left(A(\mathcal{E})^{o}\right.$.) sending some $\mathcal{F}$. to $\operatorname{Hom}^{\bullet}\left(\mathcal{E}_{\bullet}, \mathcal{F}\right.$. $)$ with the natural $A(\mathcal{E})$.-module structure. If $\mathcal{E}$ is a generator, then $L(\mathcal{E})$ and $R(\mathcal{E})$ are an inverse pair of equivalences. But in this case, it is well-known that $\mathcal{E}^{\vee}$ is also a generator, and $E \otimes_{k} \mathcal{E}^{\vee}$ is a generator for $\mathcal{D}(X \times X)$. We then have a adjoint pair of equivalences

$$
\begin{aligned}
& L\left(\mathcal{E} \otimes \mathcal{E}^{\vee}\right): \mathcal{D}\left(A(\mathcal{E})^{o} . \otimes_{k} A\left(\mathcal{E}^{\vee}\right)^{o} .\right) \rightarrow \mathcal{D}(X \times X), \\
& R\left(\mathcal{E} \otimes \mathcal{E}^{\vee}\right): \mathcal{D}(X \times X) \rightarrow \mathcal{D}\left(A(\mathcal{E})_{.}^{o} \otimes A\left(\mathcal{E}^{\vee}\right)_{.}^{o}\right) .
\end{aligned}
$$

If we identify $A\left(\mathcal{E}^{\vee}\right)^{o}$. $\cong A(\mathcal{E})$., then $L\left(\mathcal{E} \otimes \mathcal{E}^{\vee}\right)$ sends the diagonal bimodule $A(\mathcal{E})$. to the composition $\mathcal{K}\left(\mathcal{E}^{\vee}\right) \circ \mathcal{K}(\mathcal{E}) \in \mathcal{D}(X \times X)$, while the adjoint and inverse equivalence $R\left(\mathcal{E} \otimes \mathcal{E}^{\vee}\right)$ sends the structure sheaf $\mathcal{O}_{X}$ of the diagonal 
$X \subset X \times X$ to the diagonal bimodule $A(\mathcal{E})$.. Therefore $\mathcal{K}(\mathcal{E}) \circ \mathcal{K}\left(\mathcal{E}^{\vee}\right) \cong \mathcal{O}_{X}$, and this is exactly the identity morphism in $\mathcal{F} \mathcal{M}^{\text {big }}(k)$.

Lemma 4.19 has a useful corollary. Say that a framing for the 2-category $\mathcal{F} \mathcal{M}(k)$ is a choice of a generator $E \in \mathcal{D}(X)$, its $h$-injective representative $\mathcal{E}_{\text {. }}$, and a cofibrant $h$-flat termwise-flat replacement $A(\mathcal{E}) . \cong$ End $^{\bullet}\left(\mathcal{E}_{\text {• }}\right)$ for any $X \in \operatorname{Sch}(k)$.

Corollary 4.20. Any framing for $\mathcal{F M}(k)$ defines a fully faithful 2-functor $\mathcal{F} \mathcal{M}(k) \rightarrow \mathcal{D} \mathcal{M}$ or $(k)$ sending $\left\langle X, \mathcal{O}_{X}\right\rangle$ to $A(\mathcal{E})$., and two different framings define equivalent 2 -functors.

Proof. For any framing, we can take the equivalences of Lemma 4.19 and plug them into Proposition 3.7 as in Example 3.8. the resulting twisting functor $\Theta: \mathcal{F} \mathcal{M}(k) \rightarrow \mathcal{F} \mathcal{M}^{b i g}(k)$ factors through $\mathcal{D} \mathcal{M o r}(k)$ and gives the required fully faithful 2 -functor. If we have two framings, we can combine them to obtain a collection of equivalences for the 2-category $\mathcal{F} \mathcal{M}(k) \times{ }^{2}$ eq, and the twisting functor then gives an equivalence in the sense of (3.7).

\section{References}

[AL1] R. Anno and T. Logvinenko, Spherical DG-functors, J. Eur. Math. Soc. 19 (2017), 2577-2656.

[AL2] R. Anno and T. Logvinenko, $\mathbb{P}^{n}$-functors, arXiv:1905.05740.

[Be] J. Bénabou, Introduction to bicategories, Reports of the Midwest Category Seminar (Berlin, Heidelberg), Springer Berlin Heidelberg, 1967; 1-77.

[BV] A. Bondal and M. van den Bergh, Generators and representability of functors in commutative and noncommutative geometry, Mosc. Math. J. 3 (2003), 1-36.

[Bou] A.K. Bousfield, Constructions of factorization systems in categories, J. Pure Appl. Algebra 9 (1976/77), 207-220.

[DM] P. Deligne and J.S. Milne, Tannakian categories, in Hodge Cycles, Motives, and Shimura Varieties, LMN 900, Springer-Verlag, 1982; 101-228.

[G] A. Grothendieck, Catégories fibrée et descente, SGA I, Exposé VI, SMF, 2003.

[H] V. Hinich, Homological algebra of homotopy algebras, Comm. Algebra 25 (1997), 3291-3323.

[K1] D. Kaledin, Trace theories and localization, Contemp. Math. 643 (2015), 227-263.

[K2] D. Kaledin, Witt vectors, commutative and non-commutative, Russian Math. Surveys, 73 (2018), 1-30. 
[K3] D. Kaledin, Trace theories, Bökstedt periodicity and Bott periodicity, arXiv:2004.04279

[Ke] B. Keller, On differential graded categories, in International Congress of Mathematicians, Vol. II, Eur. Math. Soc., Zürich, 2006; 151-190.

[M] S. Mac Lane, Categories for the working mathematician, second ed., Graduate Texts in Mathematics, 5, Springer-Verlag, New York, 1998.

[O] D. Orlov, Smooth and proper noncommutative schemes and gluing of DG categories, Adv. Math. 302 (2016), 59-105.

[S] G. Segal, Categories and cohomology theories, Topology 13 (1974), 293-312.

Affiliations:

1. Steklov Mathematics Institute, Algebraic Geometry Section (main affiliation).

2. Laboratory of Algebraic Geometry, National Research University Higher School of Economics.

E-mail address: kaledin@mi-ras.ru 